\title{
Theoretical and experimental study of protein-lipid interactions
}

\author{
Dissertation \\ zur Erlangung des Doktorgrades \\ der Mathematisch-Naturwissenschaftlichen Fakultäten \\ der Georg-August-Universität zu Göttingen
}

vorgelegt von

Vesselka Petrova Ivanova

aus Vratza, Bulgarien

Göttingen 2000 
D 7

Referent: Prof. Reiner Kree

Korreferent: Dr. Thomas Heimburg

Tag der mündlichen Prüfung: 1.11.2000 


\section{Danksagung}

An dieser Stelle sei ganz herzlich all denen gedankt, die mich bei der Erstellung dieses Werkes unterstützt haben:

PD Dr. Thomas Heimburg,

für die gute und freundliche Betreuung und für die vielen fruchtbaren Diskussionen und Anregungen:

Prof. Reiner Kree,

für die Unterstützung dieser Arbeit von Seiten der Universität Göttingen:

Peter Grabitz,

aus meiner Arbeitsgruppe, für die wunderbaren Relaxationsmessungen;

Or. Tilman Schäfer,

aus der Abteilung Molekulare Biologie, für die freundliche Mithilfe bei den AFM. Experimenten;

Dr. Wolfgang Doster und Dr. Harald Leyser,

vom Institut für Eestkörperphysik E13, Technische Universität München, für das Ermöglichen der zeitaufgelösten kalorimetrischen Messungen und für die freundliche Unterstützung und Mithilfe;

\section{Inge Dreger,}

für das Lesen und Komigieren von meinem Manuskript und für die stete Hilfsbereitschaft;

\section{Holger Ebel und Matthias Schneider,}

ehemalige Mitglieder meiner Arbeitsgruppe, für die gute Unterstutzung und die freundschaftliche Atmosphöre;

\section{Martin Fechner,}

für die freundliche Hilfe bei Problemen mit dem PC:

Nicht zuletzt bedanke ich mich bei der Deutschen Forschungsgemeinschaft für die finanzielle Unterstutzung dieses Projektes. 


\section{CONTENTS}

Contents

$\begin{array}{ll}\text { General introduction } & 1\end{array}$

Chapter 1. Materials and methods

1.1. Differential scanning calorimetry ........................................ 11

1.2. Pressure-calorimetry ........................................................... 12

1.3. Atomic Force Microscopy ....................................................... 13

1.4. Frequency-dependent calorimetry ............................................. 15

1.5. Sample preparation ................................................................ 18

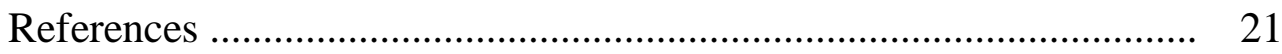

Chapter 2. Ising model of the lipid membrane and Monte Carlo simulations 23

2.1. Introduction ....................................................................... 23

2.2. Two-state model for the gel-to-fluid transition ........................... 24

2.3. Fluctuations and heat capacity ................................................. 27

2.4. Histogram method for Monte Carlo data analysis ....................... 30

2.5. Construction of a broad monolayer histogram ........................... 31

2.6. Fit of measured heat capacity profiles ....................................... 38

2.7. Discussion ........................................................................... 41

2.8. Conclusions ........................................................................... 45

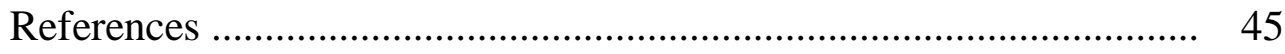

Chapter 3. Monte Carlo simulations on mixed lipid/peptide systems 49

3.1. Introduction .......................................................................... 49

3.2. Energy of the system and distribution of states .......................... 50 
3.3. Simple case of a gel-like peptide .................................................. 54

3.4. Construction of the broad four-dimensional histogram ................. 59

3.5. Model simulation results ................................................................ 60

3.5.1. Limit cases ................................................................... 60

3.5.2. Intermediate cases ......................................................... 65

3.6. Comparison with experimental heat capacity profiles ................... 68

3.7. Atomic force microscopy experiments ........................................... 74

3.8. Peptide-induced membrane fluctuations .......................................... 78

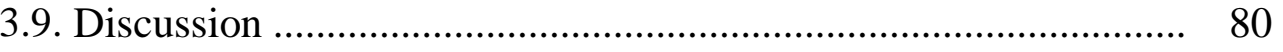

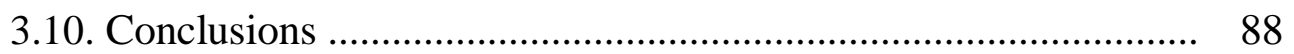

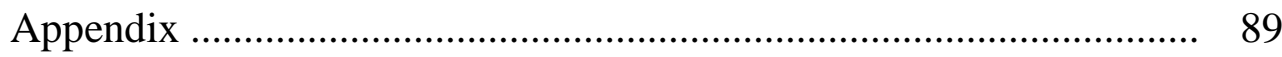

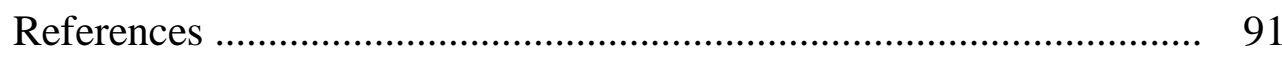

$\begin{array}{ll}\text { Chapter 4. Simulation study of the lipid kinetics } & 94\end{array}$

4.1. Introduction ........................................................................... 94

4.2. Monte Carlo simulations including a temperature-jump ............... 95

4.3. Calculation of the relaxation times in the two-state Ising model... 96

\begin{tabular}{|l|ll}
\hline 4.4. Why are the rate constants closely related to the heat capacity? & ... & 98
\end{tabular}

4.5. Experimental results and discussion ........................................... 102

4.6.1. Frequency-dependent calorimetry ................................... 102

4.6.2. Relaxation times resolved from pressure calorimetry ...... 104

4.6.3. Discussion ................................................................... 106

4.6. Conclusions ............................................................................. 108

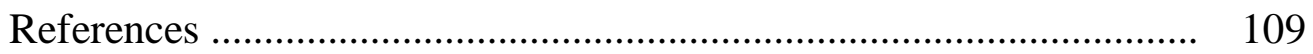

$\begin{array}{ll}\text { General conclusion } & 111\end{array}$ 


\section{General introduction}

In the design of cells and subcellular organelles, nature has developed a highly specialized technology based on membrane encapsulation. Biological membranes are essential to living organisms as they provide a selective permeability barrier and also the environment for a multitude of functional processes. The biomembranes are complex and well-organized multimolecular assemblies composed of a wide variety of protein and lipid molecular species.

Lipids are amphiphilic molecules with a polar part (hydrophilic head) and a hydrocarbon part (hydrophobic tail). Figure 1 gives a representative structure of a lipid.

\section{hydrophobic tails}

hydrophilic head

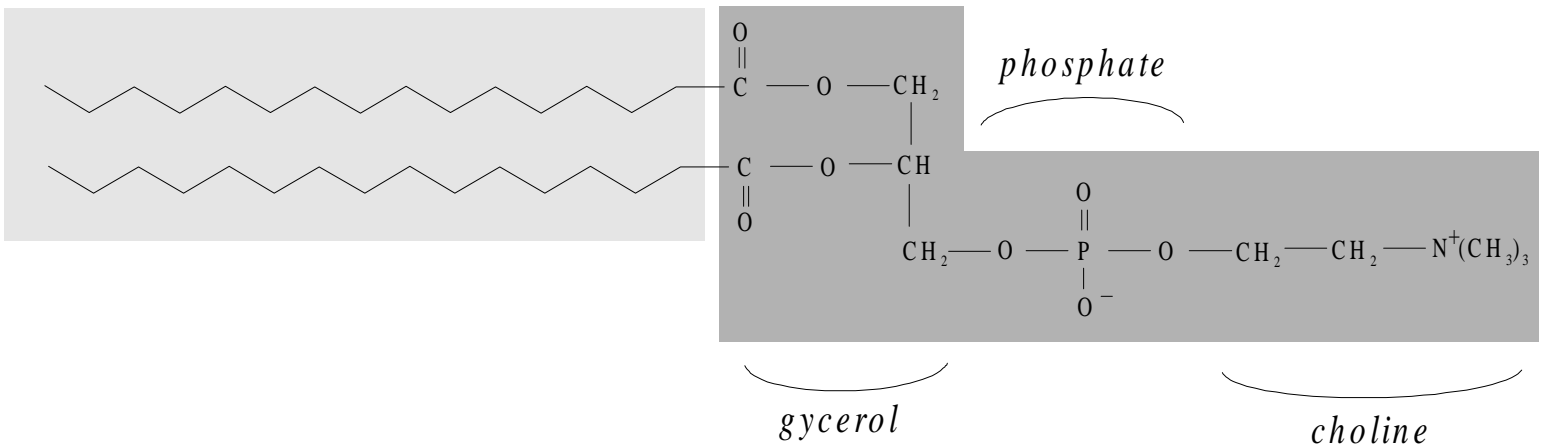

Figure 1. Chemical structure of a phospholipid (dipalmytoyl phosphatidylcholine, DPPC).

In aqueous environment lipids self-assemble spontaneously in order to protect their hydrophobic tails from contact with the water molecules. The macroscopic structure of these molecular aggregates depends on the chemical structure of the lipid as well as on the water content. Some of the variety of such molecular assemblies are shown in Figure 2.

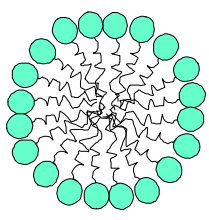

a) micelle

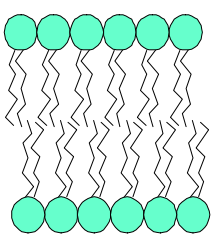

b) bilayer

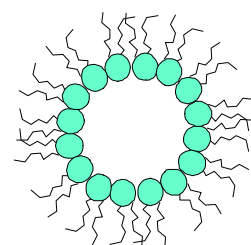

c) inverse hexagonal phase

Figure 2. Examples of phospholipid structures. 
The lipid aggregates are stabilized primarily by the hydrophobic interaction resulting from the unfavorable contact of the hydrocarbon tails with water. The hydrophobic effect arises from the ordering of water as it packs around a non-polar hydrocarbon [19]. Other stabilizing forces are van der Waal's interactions between the hydrocarbon chains, electrostatic interactions and hydrogen bonds between the polar heads. In relation to biological systems the most important structure is the lipid bilayer (Figure 2b).

Most of the current view on biological membranes is governed by the fluid-mosaic model proposed by Singer and Nicolson [18]. Within this model the lipids form a fully homogeneous bilayer to which various species of proteins are associated. A scheme of a biomembrane according to the fluid-mosaic model is shown in Figure 3. Some of the proteins are only attached to the surface of the lipid bilayer. These are called peripheral proteins. Others, the integral and the transmembrane proteins, are largely buried within the lipid matrix. The latter are exposed to both sides of the membrane.

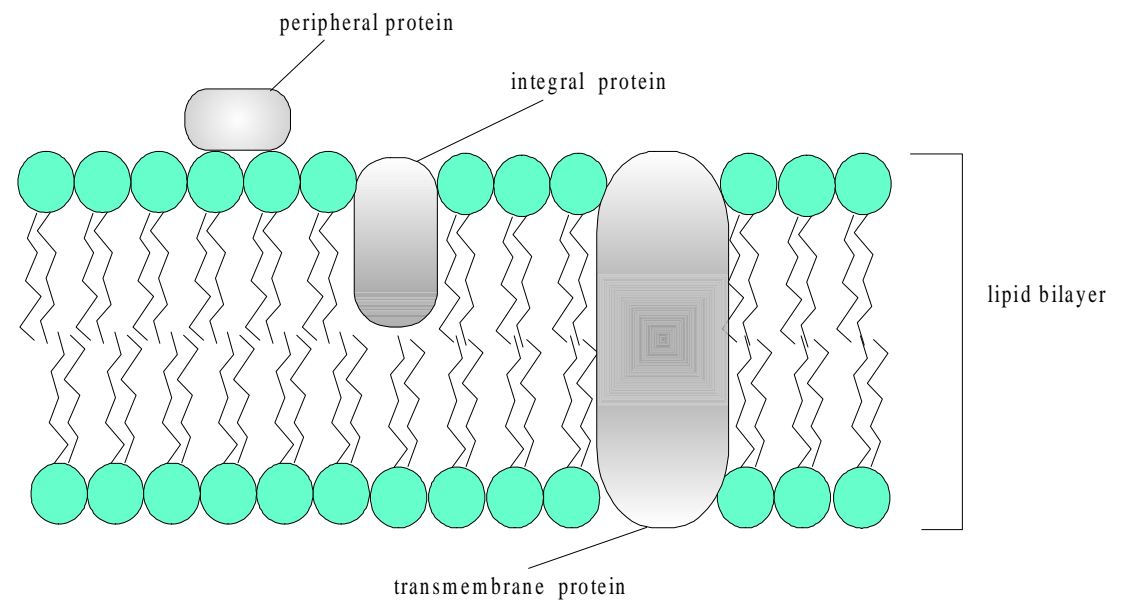

Figure 3. Scheme of a biomembrane according to the fluid-mosaic model.

The crucial property of this molecular assembly is the bilayer fluidity which assures sufficient lateral mobility of the membrane components to support biological functions. This picture is, however, an oversimplified presentation of the role of the lipids and proteins for the function of the biomembrane. For instance, the model does not account for the variety of lipids normally present in the biological systems. The reason for this diversity is not at all clear, although there is an increasing awareness of the multiple role of lipids in membranes [6]. For example, it has been observed that bacteria grown at different temperatures have different lipid composition [1]. Also a significant dependence of the 
activity of the membrane proteins $\left(\mathrm{Ca}^{2+}, \mathrm{Mg}^{2+}\right)$ ATPase and $\left(\mathrm{Na}^{+}, \mathrm{K}^{+}\right)$ATPase on the chain length of those lipids building the bilayer has been reported [3].

The biomembrane is a very complex structure with many components. Therefore, to understand the basic physical principles underlying its function, much research has been performed on model systems. A model membrane is a system which carries the essential characteristics of a biomembrane, but has less components. Such systems have been the subject of investigation in the present study.

An important feature of the single lipid membrane systems is that they undergo transitions at well defined temperatures. The most investigated is the main transition, or melting. This transition is due to the following: There is free rotation about each $\mathrm{C}-\mathrm{C}$ bond in the hydrocarbon chains with preferred energy minima at given angles - one being the absolute energy minimum (trans - conformation) and the other two having equal excitation energies (gauche $^{+}$and gauche $e^{-}$conformations). When all the $\mathrm{C}-\mathrm{C}$ bonds are in trans-conformation (all-trans), the two chains are parallel and are maximally extended. However, the probability for the occurrence of a gauche-bond increases with increasing temperature. At high temperatures, the chains are not correlated and are fully disordered. The membrane fluidity - a measure of the bilayer lateral viscosity - drops by about two orders of magnitude upon membrane melting [6]. Therefore, the ordered low-temperature lipid phase is called gel phase, and the highly disordered phase is called fluid phase. Figure 4 illustrates the bilayer melting transition.

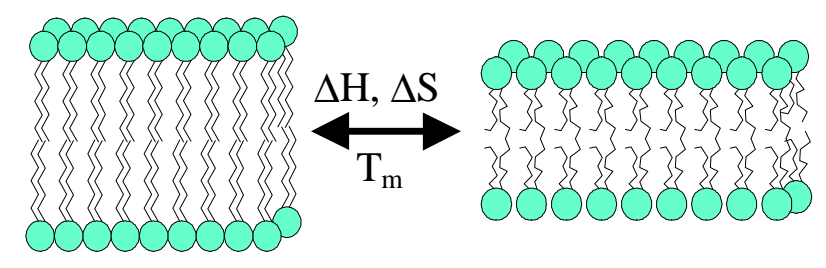

Figure 4. Scheme of the melting transition in a lipid bilayer.

The chain-melting (or gel-to-fluid) transition is an endothermic process with a relatively large heat $(8700 \mathrm{cal} / \mathrm{mol})^{*}$. The entropy changes by about $26 \mathrm{cal} / \mathrm{mol} \cdot \mathrm{K}^{*}$, the membrane volume increases $(4 \%)^{*}$ and the bilayer thickness decreases $(-16 \%)^{*}$. The heat capacity is defined as the amount of heat required to raise the temperature of the system by a given

\footnotetext{
* Data for DPPC, from [8]
} 
temperature increment $\left(C_{p}=\Delta Q / \Delta T\right)$. A typical heat capacity profile of a lipid system undergoing melting transition is given in Figure 5.

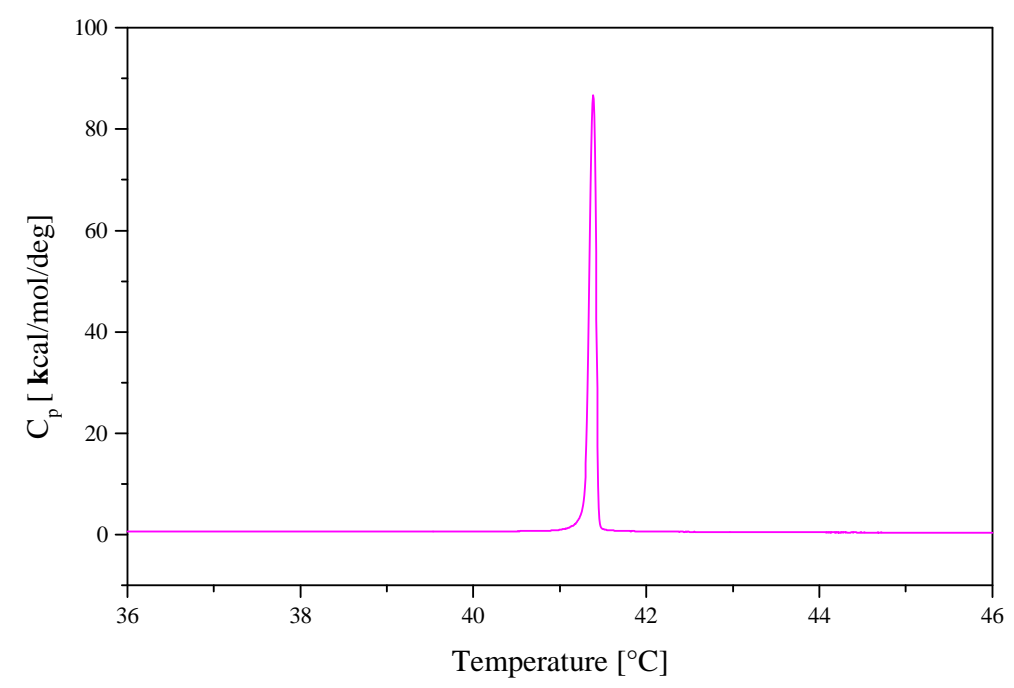

Figure 5. Heat capacity profile of a DPPC aqueous dispersion.

It is evident that the transition half-width is very small (less than $0.1 \mathrm{deg}$ ). Supposing that each lipid in the system melts independently, the calorimetric heat in a two-state transition is equal to the van't Hoff heat, and the transition half-width should be about $60 \mathrm{deg}$. [6]. This is the reason for calling the melting transition cooperative. The cooperativity implies that the lipid molecules do not undergo a melting transition independently but in a correlated manner. However, the lipid melting cannot generally be classified as a first-order phase transition. All thermodynamic properties, like membrane area, volume and thickness, vary in a continuous though dramatic fashion during transition [11]. This has led to the proposal that the lipid melting transition is pseudo-critical, i.e., in principle of first order but very close to a critical point and consequently strongly dominated by thermal fluctuations [11]. These thermal fluctuations may induce states of dynamic lateral heterogeneity in the membrane plane $[11,12,14]$. The formation of clusters composed of lipids in a single state can partially be related [14] to the difference of the hydrophobic thickness of the gel and the fluid bilayer. The disordered fluid chains are thus effectively shorter than the fully ordered chains in the gel phase. Hence, in order to protect its hydrophobic parts from the water molecules each lipid tends to surround itself with lipid chains of a similar length [13]. Membrane heterogeneity has been detected experimentally 
in model systems $[3,10,16]$ as well as in biomembranes $[17,4]$. It is important to stress at this point that for a pure one-component system in thermal equilibrium, Gibbs's phase rule forbids coexistence of phases over a finite temperature range. Such phenomena are, therefore, strictly non-equilibrium effects.

In addition, the increased thermal fluctuations in the melting transition region and the resulting membrane heterogeneity have been related to an enhanced compressibility and elasticity of the bilayer [8] as well as to an increased permeability of water and small ions $[2,11]$.

The equilibrium lipid fluctuations are dynamic phenomena and an important aspect of their character is the time scale over which they occur and how various membrane components affect these times. The lipid melting transition is associated with some interesting and unusual kinetics. It has been reported [20] that close to the gel-to-fluid transition the lipid relaxation following an external distortion becomes very slow and the characteristic times of this kinetics are in the range of seconds. These long relaxation times have been suggested as being cooperative processes involving a large number of molecules via cluster formation and cluster melting rather than due to single-molecule effects [20].

In mixed lipid-protein systems it turns out that the function of some incorporated integral proteins is significantly influenced by the bilayer thickness [2-4,9,12,14,21]. One of the important elements of the lipid-protein interaction hence involve hydrophobic matching [13] leading to lipid sorting and lipid selectivity at the protein-lipid interface. This theoretical concept is illustrated in Figure 6.

Thus, the lipid conformational structure and the lateral organization of the different lipid components are likely to adjust to the presence of the protein. On the other hand, the lateral membrane organization (in terms of fluctuating lipid clusters) will be affected by the presence of proteins and will influence the lateral organization and distribution of proteins [7]. However, these theoretical considerations need to be tested in experiments. 


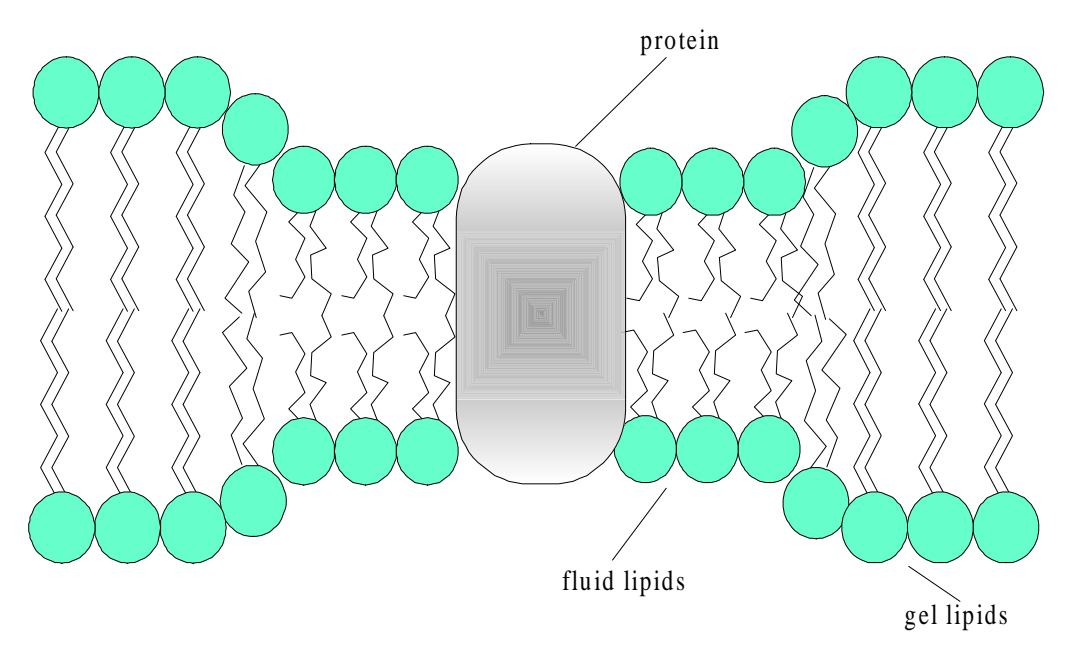

Figure 6. Schematic illustration of the principle of protein/lipid hydrophobic matching. The protein is surrounded by lipids with a similar hydrophobic length.

The thermodynamics of the melting transition can be described approximately in terms of individual lipid molecules existing in two distinct states [5], gel and fluid. This does not imply, however, that a membrane exists in only two states. The membrane can exist in a series of intermediate states which consist of mixtures of phospholipid molecules in the two different conformations. The membrane melting is a very cooperative process and the large number of molecules involved implies a principle difficulty for a theoretical description of such phenomena based on molecular microscopic interaction models. Even in the simple two-state model there are $2^{\mathrm{N}}$ possible micro-states, where $\mathrm{N}$ is the number of lipid molecules in the system $(\mathrm{N} \approx 11)$. Only for very simplified models it is possible to perform any analytically exact calculations [15]. The phenomenological Landau-type models have implicitly built in that they are solved using a self-consistent mean-filed-type calculation. By nature this type of calculation is very approximate by its suppression of thermal fluctuations and thus gives at best only qualitative description of the properties of the membrane in the transition region. The second type of calculations are based on modern computer simulation techniques which are used to solve the statistical mechanical problems of systems consisting of many particles. These techniques include Monte Carlo simulation methods which exploit various types of stochastics sampling methods and Molecular Dynamics simulation methods which solve the dynamical equations of motion in a way which is numerically exact. In the present thesis the Monte Carlo simulation approach was applied. This numeric technique although time consuming allows to account for the thermal 
fluctuations in the lipid membrane at equilibrium and may be considered an experiment on a well-defined system carried out under completely controlled conditions.

The aim of the present thesis is to investigate both theoretically and experimentally the interactions of integral proteins with lipid membranes. Since from the heat capacity profile the whole thermodynamic information about the investigated (homogeneous) system, can, in principle, be deduced, we performed very precise calorimetric measurements on pure lipid and mixed lipid-peptide systems. The theoretical analysis is based on the two-state Ising model, adopted for the lipid melting transition. In order to account properly for the enthalpy and volume fluctuations which accompany the gel-to-fluid transition we applied the Monte Carlo simulation method. Within this approach, the heat capacity is calculated from the enthalpy fluctuations generated during a Monte Carlo simulation. However, the main disadvantage of the Monte Carlo technique are the very time-consuming calculations. Therefore, we used a method called histogram method, with which we were able to perform a fast quantitative comparison between calculated and experimentally measured heat capacity profiles. This histogram technique allows a quasi-analytical determination of the heat capacity traces of pure lipid systems and lipid-peptide mixtures. Thus, the unusual shapes of experimentally measured heat capacity profiles of lipid bilayers incorporating peptides are analyzed with the aim of resolving peptide aggregation properties. The predictions of the heat capacity analysis are compared to experimental data obtained with atomic force microscopy technique.

The thesis is organized as follows:

In Chapter 1, the basic experimental methods used are described in some detail. Information is given about the model systems and the sample preparation.

Chapter 2 presents a Monte Carlo simulation study on a single-component lipid system. The two-state Ising model adopted for the lipid melting transition and the MC simulations as well as the histogram technique for MC data analysis are described here. Since only a small part of the phase space is explored by a single MC simulation, histograms produced at different sets of parameters have to be combined to construct a broad histogram of 
degeneracy of states. In this chapter we describe the matching procedure used for this purpose. Further, the broad histogram was used in order to fit experimental heat capacity profiles.

In Chapter 3, the two-state Ising model is extended in order to describe a lipid membrane containing small integral peptides. Considering the case of a constant peptide fraction, the energy of the system depends on four variables - the number of fluid lipids and the number of the unlike nearest neighbor contacts, i.e., gel-fluid, gel-peptide and fluid-peptide. Therefore, a broad four-dimensional histogram has to be constructed for a mixed lipidpeptide system. This problem is computationally more demanding than that described in Chapter 2. The four-dimensional histogram is then used for quasi-analytical calculation of heat capacity profiles in order to compare with DSC traces measured on some model systems. As a consequence, the peptide aggregation properties in gel and fluid lipid phase are predicted. These predictions are tested in atomic force microscopy measurements. Additionally, the simulations demonstrate how the different mixing of peptides in both lipid phases can induce fluctuations in the membrane plane.

Chapter 4 is devoted to the investigation of the lipid kinetics close to chain-melting transition. From the decay of the enthalpy fluctuations generated in Monte Carlo simulations, we calculated relaxation times which display very close relation to the heat capacity. In order to justify this finding we developed a theory on deriving the rate constants from the broad histogram of distribution of states. The experimental study of the lipid relaxation involves specific heat spectroscopy measurements and pressure-calorimetry measurements. While the first allow only an estimation of the characteristic time-scale of the decay of the enthalpy fluctuations in lipid dispersions, the latter provide very precise data which are in very good agreement with the theoretical predictions.

The last part of the present thesis is a general conclusion which summarizes the principal results and presents some prospects for future work. 


\section{References}

[1] Alberts, B., Bray, D., Lewis, J., Raff, M., Roberts, K., and Watson, J. D. (1994). Molecular Biology of the Cell. New York, London: Garland Publishing, 3. Edition.

[2] Boheim, G., Hanke, W., and Eibl, H. J. (1980). Proc. Natl. Acad. Sci. USA, Vol. 77, 3403-3407

[3] Dumas, F., Lebrun, M.-C., and Tocanne, J.-F. (1999). FEBS Letters, Vol. 458, 271277

[4] Dumas, F., Tocanne, J.-F., Leblanc, G, and Lebrun, M.-C. (2000). Biochemistry, Vol. 39, 4846-4854

[5] Freire, E. and Biltonen, R. L. (1978). Biochimica et Biophysica Acta, Vol. 514, 54-68

[6] Gennis, R. B. (1989). Biomembranes. In Molecular Structure and Function, SpringerVerlag, New-York

[7] Gil, T., Ipsen, J. H., Mouritsen, O. G., Sabra, M. C., Sperotto, M. M., and Zuckermann, M. J. (1998). Biochimica et Biophysica Acta, Vol. 1376, 245-266

[8] Heimburg, T. (1998). Biochimica et Biophysica Acta, Vol. 1415, 147

[9] Heimburg, T. and Marsh, D. (1996). in Biological Membranes: A molecular perspective from computation and experiment, edited by K. M. Merz and B. Roux (Birkhäuser, Boston), pp. 405-462

[10] Korlach, J., Schwille, P., Webb, W. W. and Feigenson, G. W. (1999). Proc. Natl. Acad. Sci. USA, Vol. 96, 8461-8466

[11] Mouritsen, O. G. (1991). Chemistry and Physics of Lipids, Vol. 57, 179-194

[12] Mouritsen, O. G. and Biltonen, R. L. (1992). In Protein-Lipid Interactions, edited by A. Watts, Elsevier, New York, 1-35

[13] Mouritsen, O. G. and Bloom, M. (1984). Biophysical Journal, Vol. 46, 141-153

[14] Mouritsen, O. G. and Sperotto, M. M. (1992). In Thermodynamics of cell surface receptors, M. Jackson, editor, CRC Press, Boca Raton, FL. 127-181 
[15] Nagle, J. F. (1980). Annual Reviews of Physical Chemistry, Vol. 31, 157

[16] Nielsen, L. K., Bjornholm, T., and Mouritsen, O. G. (2000). Nature, Vol. 404, 352

[17] Simons, K. and Ikonen, E. (1997). Nature, Vol. 387, 569-572

[18] Singer, S. J. and Nicolson, G. L. (1972). Science, Vol. 175, 720-731

[19] Tanford, C. (1980). The hydrophobic effect: Formation of micelles and biological membranes, A Wiley-Interscience Publications, New York

[20] van Osdol, W.W., Biltonen, R. L., and Johnson, M. L. (1989). Journal of Biochemical and Biophysical Methods, Vol. 20, 1-46

[21] Zhang, Y.-P., Lewis, R. N. A. H., Hodges, R. S., and McElhaney, R. N. (1995), Biochemistry, Vol. 34, 2362-2371 


\section{Materials and methods}

\subsection{Differential scanning calorimetry}

Differential scanning calorimetry (DSC) is a technique of primary importance for obtaining information about the thermodynamics of model membranes and biomembranes. It is used to monitor and characterize changes in physical state in lipid membranes and also to characterize the perturbations of pure lipids by the interactions with other materials, such as other lipids, proteins, ions or small hydrophobic molecules. Highly sensitive instruments allow one to use samples of dilute aqueous suspensions of lipids (e.g. $<1 \mathrm{mg} / \mathrm{ml}, 1 \mathrm{ml}$ sample volume). DSC reports the following parameters:

1. Transition midpoint, $\mathrm{T}_{\mathrm{m}}$ : where the transition is $50 \%$ complete.

2. Transition enthalpy, $\Delta \mathrm{H}$ : the actual heat required for the entire transition normalized per mole or per unit weight.

3. Heat capacity, $\mathrm{C}_{\mathrm{p}}$ : the amount of heat (per gram or per mole) required to raise the temperature of the sample by a given temperature increment.

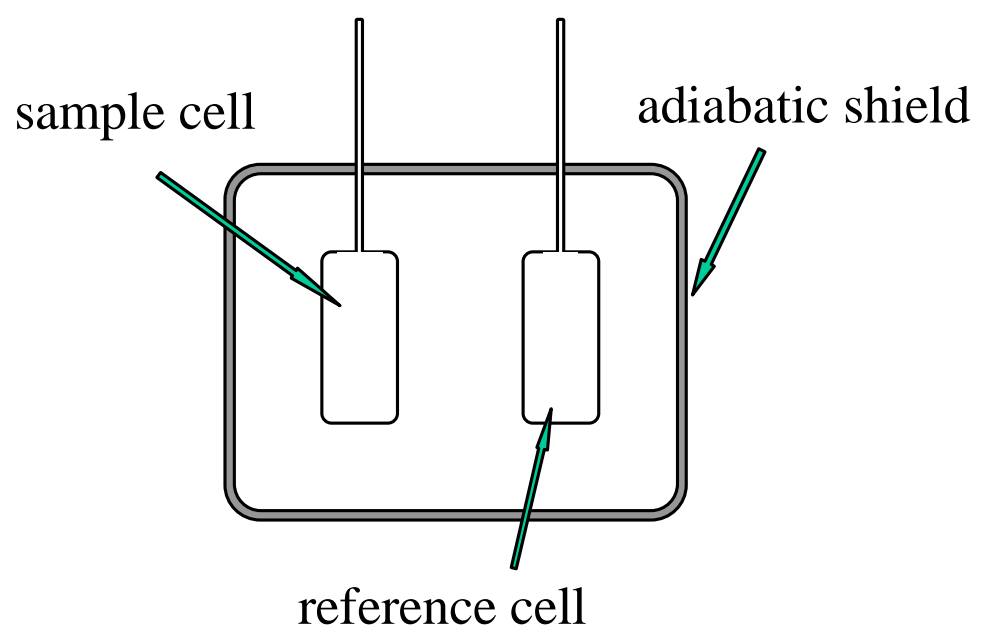

Figure 1.1. Basic construction of a differential scanning calorimeter (DSC).

A differential scanning calorimeter consists of two cells, one containing sample and the other: an inert reference material, which can be heated at a programmed rate by heaters controlled to maintain the temperature difference between the cells at zero. If the sample is a solution or a suspension, the reference material is the corresponding solvent. When a thermally initiated process takes place in the sample cell, the control system responds by 
increasing or reducing the heat supply to the sample cell so that its temperature is equal to that of the reference cell. The data output of the calorimeter is the corresponding excess power, presented as function of the temperature. It is worth noting that a heat evolution in the sample can only be observed if the thermally induced reaction is kinetically limited as compared to the heating rate of the scan [11], because a temperature increase shifts the thermodynamic equilibrium in an endothermic direction.

For our measurements a very sensitive differential scanning calorimeter VP-DSC from MicroCal (Northhampton/MA, USA) was used. The device records the excess power $\Delta P$,

the time $t$ and the temperature $\hat{T}$. Then the excess heat, $\Delta Q$, required for the temperature change and the heat capacity difference, $\Delta C_{P}$, (at constant pressure $p$ ) between the sample and the reference are computed.

$$
\begin{aligned}
& \Delta Q=\int_{t}^{t+\Delta t} \Delta P\left(t^{\prime}\right) d t^{\prime} \cong \Delta P \cdot \Delta t \\
& \Delta C_{p}:=\left(\frac{\partial Q}{\partial T}\right)_{p} \cong \frac{\Delta Q}{\Delta \hat{T}}=\frac{\Delta P}{\left(\frac{\Delta \hat{T}}{\Delta t}\right)}
\end{aligned}
$$

where $(\Delta \hat{T} / \Delta t)$ is the scan rate. The two cells are made of tantalum. The calorimeter used has a wide spectrum of scan rates $(0 . .60 \mathrm{deg} / \mathrm{h})$, a large signal-to-noise ratio and a very stable base line. The two latter features allowed, in 1997 measurements which were about ten times better than the published data obtained with other DSCs [12].

The very sensitive and at the same time robust controlling technique has allowed the construction of a pressure cell, as described in the next section.

\subsection{Pressure calorimetry}

The pressure cell for our calorimeter was built for studying the pressure dependence of the phase transition in lipid membranes. Figure 2.1 shows a scheme of the experimental set-up. High pressure acts on the lipid phase transition only as a temperature shift, e.g. a pressure of 200 bar shifts the lipid phase transition by about 5 deg. [3]. From the proportionality relation between volume and enthalpy changes close to the chain-melting transition it can 
be shown that $\Delta V_{i}=\gamma_{v o l} \Delta H_{i}$ for each micro-state $i$ of the membranous system [8]. Then for the heat capacity measured at pressure $p$ and temperature $T$, it follows that $C_{p}(T)=C_{p_{0}}\left(T^{*}\right)$, where $T^{*}=T /\left(1+\Delta p \cdot \gamma_{v o l}\right)$ and $\Delta p=\left(p-p_{0}\right)[3]$

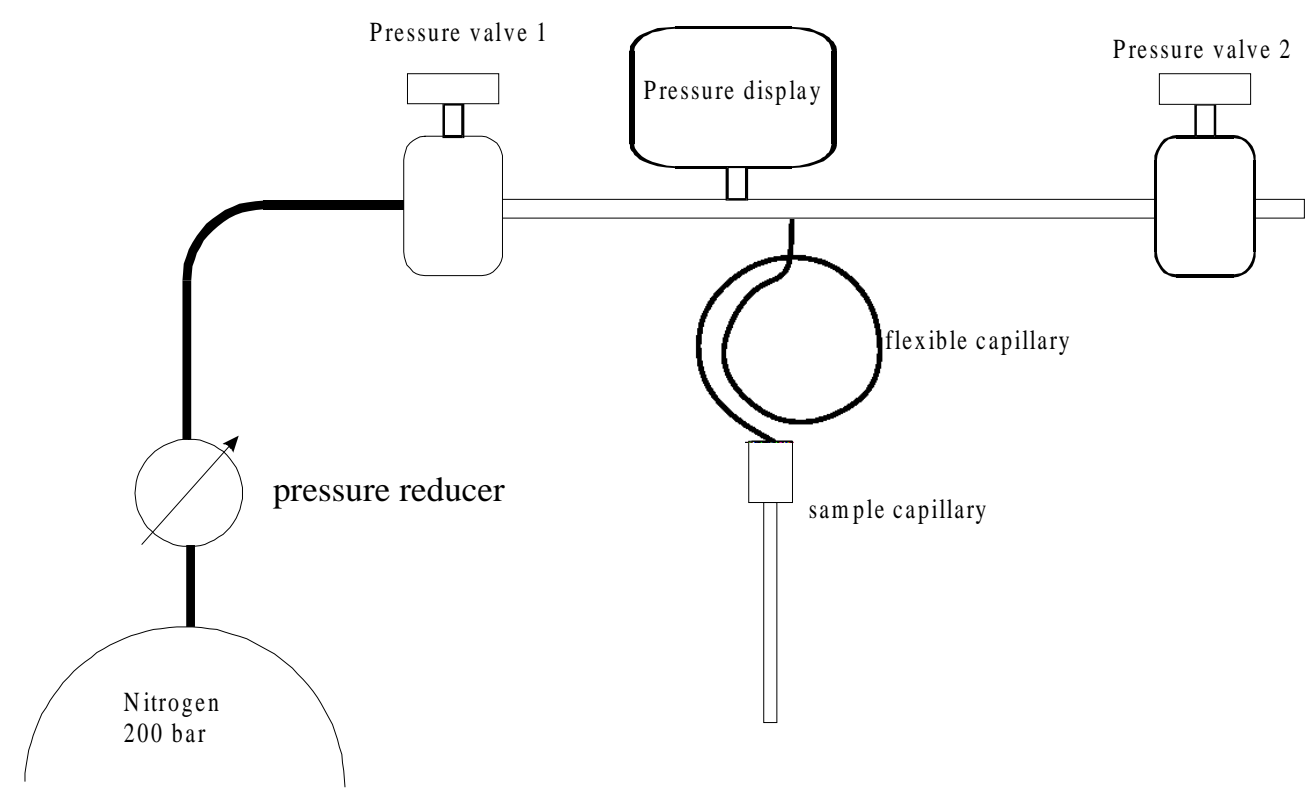

Figure 1.2. Scheme of the pressure cell set-up for the differential scanning calorimeter VP-DSC. The sample is filled into a capillary, which is then put into the sample cell of the calorimeter. The pressure valves 1 and 2 serve for rough and fine pressure adjustment, respectively. Details about the construction are given in $[3,7]$.

Thus, using the calorimeter as a thermostat, one can drive the sample into or out of the transition region by the pressure control alone. Then the time dependence of the excess power, which is required for keeping a constant temperature in the sample cell by heating or cooling it, was recorded and analyzed. The decay profiles were fitted with one- or twoexponential functions in order to obtain relaxation rates.

\subsection{Atomic Force Microscopy}

A scheme of an atomic force microscope is shown in Figure 1.3. The sample is mounted on a piezo ceramic which can be moved extremely accurately in the $\mathrm{x}, \mathrm{y}$ and $\mathrm{z}$ directions. The sample is then rastered in the $\mathrm{x}$ and $\mathrm{y}$ directions under a sharp tip. This tip is mounted at the free end of a cantilever (as shown in Figure 1.3) onto which a laser beam is focused. The 
beam is reflected from the back of the cantilever to a set of photosensitive diodes. These act to detect any deflection of the laser beam arising from the cantilever moving as the sample is rastered. A feedback loop then makes the piezo move in the $\mathrm{z}$ direction taking the laser beam back to its original position. In this way the sample is scanned with a constant force and the resulting $\mathrm{z}$ motion of the piezo produces a topographical map of the region scanned.

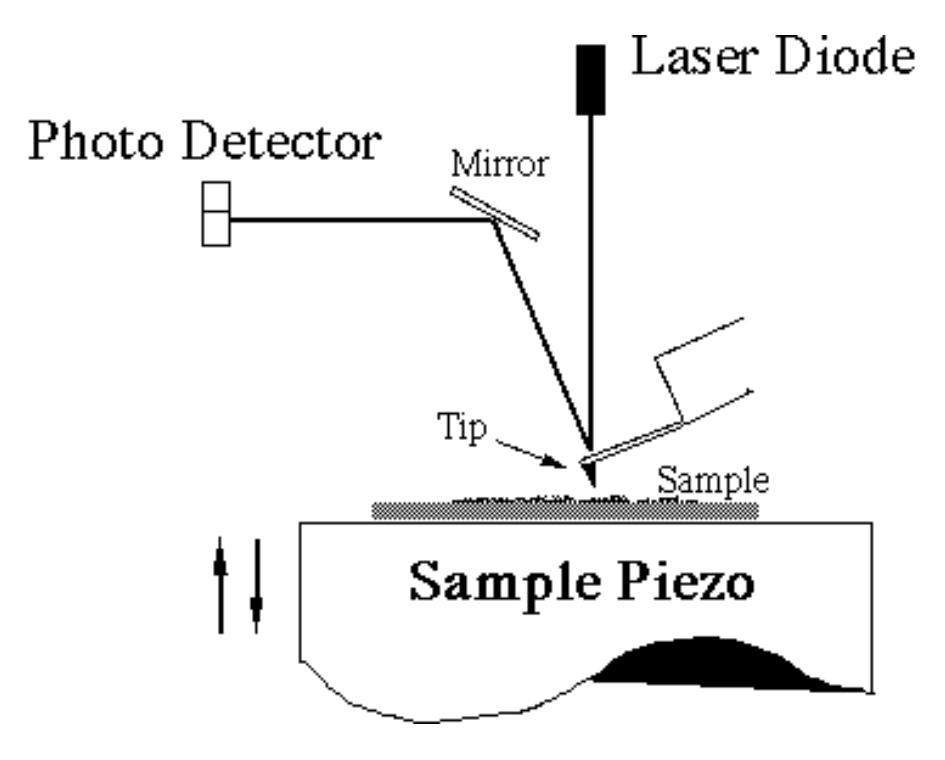

Figure 1.3. Scheme of an AFM set-up.

Our samples were imaged in water with a Nanoscope III atomic force microscope (Digital Instruments, Santa Barbara, CA). Cantilevers with nominal spring constants between 0.06 and $0.32 \mathrm{~N} / \mathrm{m}$ and oxide-sharpened silicon nitride tips (Digital Instruments) were used in contact mode. Line scan frequencies were between 2 and $8 \mathrm{~Hz}$. The images are unfiltered except for slope removal along each scan line to level the image, i.e., since there is always a drift during an AFM scan, each scanning line has a certain slope which is subtracted in the image analysis. This is a standard procedure and, in the case of a relatively homogenous scanning plane, does not have significant influence on the measured heights. 


\subsection{Frequency-dependent calorimetry}

With the common differential scanning calorimetry method (see Section 1.1) the temperature of the sample is changed over time $t$ at a constant rate $d T / d t$ while the required power $d Q(t) / d t$ is recorded. This experiment measures the specific heat of the sample: $d Q(t) / d t=C_{p}(d T / d t)$. In many cases, $C_{p}$ is time-independent and the experiment provides the true thermodynamic specific heat. However, structural transitions such as lipid melting or folding-unfolding processes in proteins involve slow relaxation processes. Thus, if the scanning rate is too fast, the system no longer adjusts adiabatically to the temperature changes and the specific heat becomes time dependent. This nonstationary specific heat describes the enthalpy relaxation of the system and contains information on the mechanism of structural transitions and intermediate states. In order to study slow relaxation processes in liquids Birge and Nagel [1,2] proposed the specific heat spectroscopy method (or frequency-dependent calorimetry method). This technique allows to derive the product of thermal conductivity and a frequency-dependent specific heat $C_{p}(\omega)$, which characterizes the enthalpy relaxation of the liquid. Figure 1.4 shows the experimental set-up scheme [9].

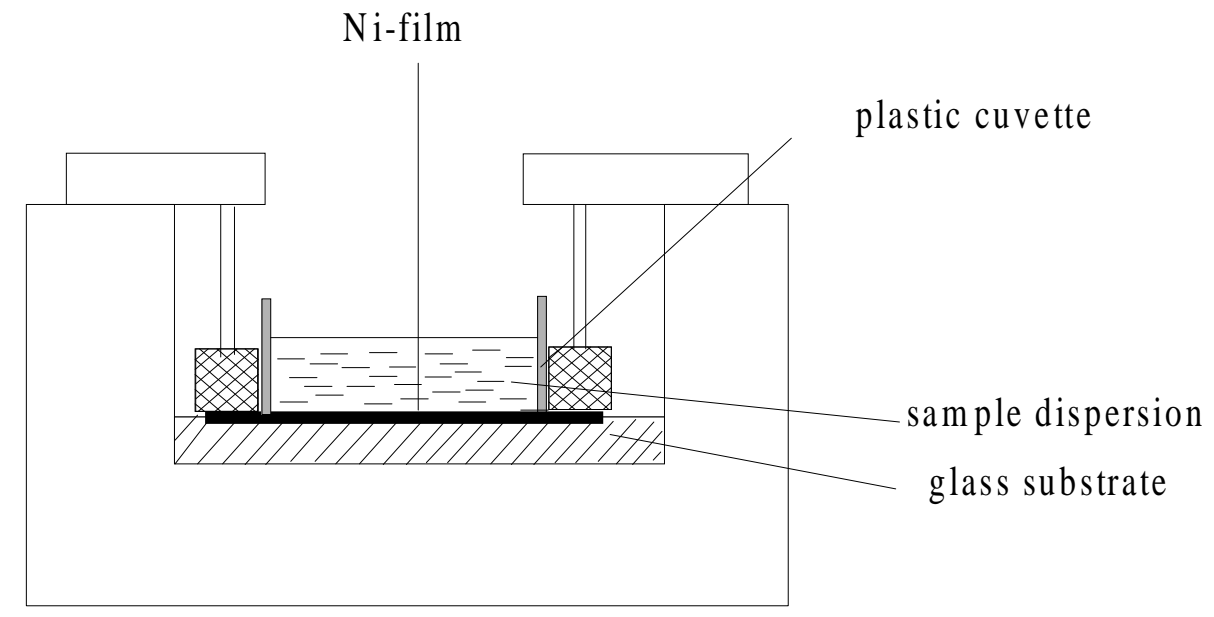

Figure 1.4. Scheme of the frequency-dependent calorimeter. An alternating current is passed through a thin nickel film $(500 \AA)$ evaporated onto a glass substrate. The film heats the sample at a defined frequency and simultaneously serves as a thermometer recording the temperature oscillations at the film-sample interface. Details about the detection electronics are given in $[10,13]$.

In frequency-dependent calorimetry a thin rectangular film is evaporated onto a substrate of window glass and is immersed into the liquid to be examined. When a sinusoidal current is 
passed through the film, $I(t)=I_{0} \cos \frac{1}{2} \omega t$, a Joulean heat proportional to the square of the current is produced, which has a component which is time-constant and one that oscillates with currency $\omega$ :

$$
Q(t)=\frac{1}{2} R I_{0}^{2}(1+\cos \omega t)
$$

This heat flows into both the window glass substrate and the sample. While the constant source leads to a static temperature gradient across the two bulks, the harmonic one creates an exponentially damped temperature wave in each of them (real part solution). This result is obtained by solving the one-dimensional heat diffusion equation with the heat source at $x=0$ and the glass substrate (index 1) and sample (index 2) at $x<0$ and $x>0$, respectively:

$$
\Delta T(x, t)=\Re\left\{\Delta T_{x=0} e^{i(\omega t-\pi / 4)} e^{k_{j} x}\right\}
$$

with

$$
k_{j}= \pm(1+i) \sqrt{\frac{\omega c_{p j} \rho_{j}}{2 \kappa_{j}}}
$$

Here $\rho_{j}, c_{p j}$ and $\kappa_{j}$ denote the mass density, the specific heat and the thermal conductivity, respectively. The wavelength is given by $\lambda_{j}=1 / k_{j}$ and varies as $1 / \sqrt{\omega}$. The one dimensional treatment of the problem is justified, if this wavelength is considerably smaller than the lateral dimensions of the film.

For the temperature oscillations at the nickel film, i.e., $x=0$, one obtains [13]:

$$
\Delta T_{x=0, t}=\frac{Q_{0}}{\sqrt{\omega}\left(\sqrt{\varepsilon_{1}}+\sqrt{\varepsilon_{2}}\right)} \cdot \frac{1}{2} \cos \left(\omega t-\frac{1}{4} \pi\right)
$$

where $\varepsilon_{j}=c_{p j} \rho_{j} \kappa_{j}$ is the so-called effusivity and $Q_{0}=R I_{0}^{2}$ is the amplitude of the oscillating heat source. The experiment thus yields the product of specific heat and thermal conductivity. The heat source can simultaneously serve as a thermometer as the resistance of the Ni-film depends on the temperature according to $R=R_{0}(1+\alpha T)$. The latter holds for small temperature changes. The temperature oscillations thus induce a time-dependent component in the resistance: 


$$
\Delta R(t)=\alpha R_{0} \Delta T_{x=0, t}
$$

According to Ohm's law the initial current experiences an additional voltage drop across the oscillating resistance:

$$
\Delta U(t)=I(t) \cdot \Delta R(t)=\frac{\alpha I_{0} Q_{0} R_{0}}{\sqrt{\omega}\left(\sqrt{\varepsilon_{1}}+\sqrt{\varepsilon_{2}}\right)} \cdot \frac{1}{4} \cdot\left[\cos \left(\frac{3}{2} \omega t-\frac{\pi}{4}\right)+\cos \left(\frac{1}{2} \omega t-\frac{\pi}{4}\right)\right]
$$

The latter consists of two components - one oscillating with frequency $3 \omega / 2$ and the other - with $\omega / 2$. Since the $\omega / 2$-component is present even in the absence of temperature oscillations $\left(I(t)=\frac{1}{2} I_{0}(1+\cos \omega t)\right)$, it is the $3 \omega / 2$-component which is measured to get information about the heat diffusion properties of the substrate and the sample.

The thermal effusivity, $\varepsilon_{1}$, of the glass substrate is determined in a measurement without sample, i.e. the heating film is in contact with air. In this case $\varepsilon_{2} \approx 0$, because the density of air is much smaller than the density of the glass substrate. Thus, for $\varepsilon_{1}$ one obtains

$$
\varepsilon_{1}=\left(\frac{\alpha I_{0} Q_{0} R_{0}}{4 \cdot \sqrt{\omega} \cdot \Delta U_{\frac{3 \omega}{2}{ }^{(e m p t y)}}}\right)
$$

where $\Delta U_{\frac{3 \omega}{2}}^{{ }^{(e m p t y)}}$ is the amplitude of the $3 \omega / 2$-signal in the measurement without sample. Generally, $\varepsilon_{1}$ depends on the temperature, but in a small temperature interval it can be considered as a constant. Then, for the effusivity of the sample one has

$$
\varepsilon_{2}=\varepsilon_{1}\left(\frac{\Delta U_{\frac{3 \omega}{2}}^{\left({ }^{(e m p t y)}\right.}}{\Delta U_{\frac{3 \omega}{2}}}\right)^{2}
$$




\subsection{Sample Preparation}

\section{Lipids}

The lipids used are described briefly in the table below.

\begin{tabular}{|c|c|}
\hline DMPC & 1,2-dimyristoyl-sn-glycero-3-phosphocholine \\
$677.94 \mathrm{~g} / \mathrm{mol}$ & dimyristoylphosphatidylcholine \\
\hline DPPC & 1,2-dipalmitoyl-sn-glycero-3-phosphocholine \\
$734.05 \mathrm{~g} / \mathrm{mol}$ & dipalmitoylphosphatidylcholine \\
\hline
\end{tabular}

Table 1.1 Abbreviation, systematic name, molar mass and a common synonym of the lipids used. Molecular structure of DPPC is given in Figure 1. in the general introduction. The hydrophobic chains of DMPC are two $\mathrm{CH}_{2}$-groups shorter than those of DPPC.

The lipids were purchased as powder from Avanti Polar Lipids (Birmingham, AL) and used without further purification. Vesicles were prepared in a buffer $(5 \mathrm{mM}$ Hepes, $1 \mathrm{mM}$ EDTA, pH 7.5) with lipid concentration of about $10 \mathrm{mM}$. For the kinetic measurements, however, dispersions with lipid concentration of about $100 \mathrm{mM}$ were used, to get better signal-to-noise ratio

\section{Vesicle dispersions}

Multilamellar vesicles (MLV) form spontaneously by dispersing the lipid in buffer and gentle shaking above melting temperature. Small unilamellar vesicles (SUV) were prepared by ultrasonication with 50 Watts for several minutes using a Model W185 sonifier from Heat System-Ultrasonics (Plainview, N.Y.). Since SUVs are unstable below melting temperature, the respective calorimetric scans were performed in the down scan mode (cooling from high to low temperatures). Gradually, small vesicles spontaneously fuse into large unilamellar vesicles (LUVs). The size-distribution of SUVs obtained in lightscattering measurements is given in Figure 1.5. To prepare LUVs, SUVs were stored in a refrigerator at $4^{\circ} \mathrm{C}$ for about two weeks. 
Another way to prepare large unilamellar vesicles is the extrusion method. In this case a dispersion of MLVs is pressed several times through a filter with a pore size of about 100 $\mathrm{nm}$. Since the MLVs are too large to go through (diameter about $500 \mathrm{~nm}$ ), they are destroyed and form LUVs.

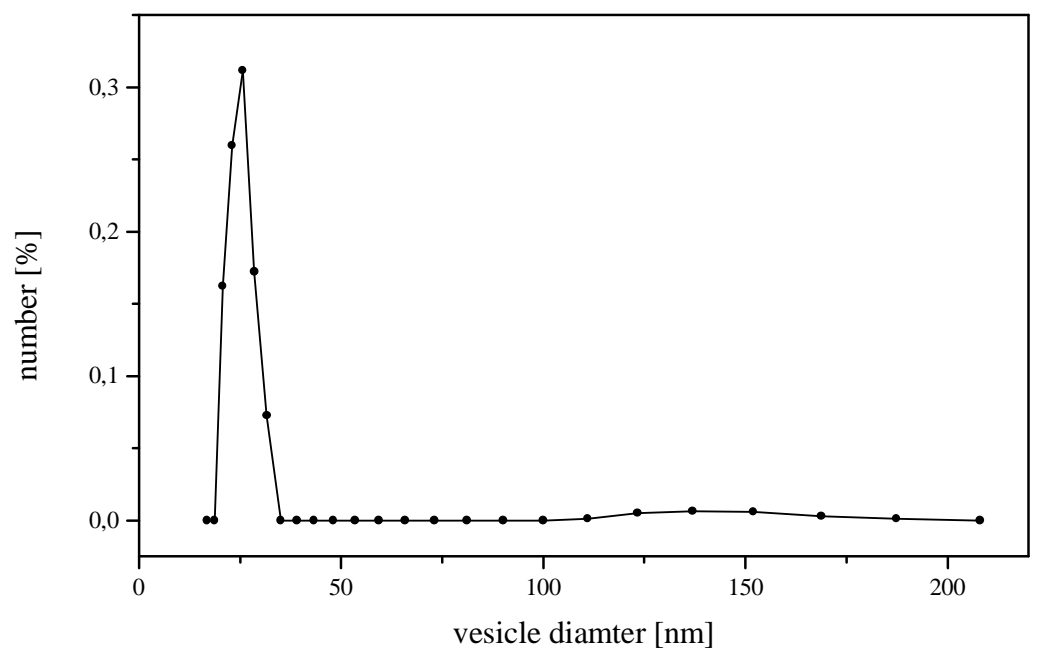

Figure 1.5. Normalized size-distribution of small unilamellar vesicles of DPPC as obtained in dynamic light-scattering experiments*. The measurements are performed at temperature $T=50^{\circ} \mathrm{C}\left(>T_{m}\right)$ immediately after sonification. Mean diameter of SUV $25 \mathrm{~nm}$. The second peak (with intensity <2\%) is due to LUV mean diameter $140 \mathrm{~nm}$.

\section{Gramicidin A}

The peptide antibiotic gramicidin A is one of the best characterized and most extensively studied membrane-associated peptides. It is a linear hydrophobic polypeptide with the following structure

HCO-L-Val1-Gly2-L-Ala3-D-Leu4-Ala5-D-Val6-L-Val7-D-Val8-L-Trp9-

$$
\text { D-Leu }{ }^{10}-\mathrm{L}-\operatorname{Trp}^{11} \text {-D-Leu }{ }^{12}-\mathrm{L}-\operatorname{Trp}^{13} \text {-D-Leu }{ }^{14}-\mathrm{L}-\mathrm{Trp}^{15}-\mathrm{NHCH}_{2} \mathrm{CH}_{2} \mathrm{OH}
$$

Gramicidin can adopt a variety of conformations. For us, the channel forming conformation - single-stranded N-N terminal helical dimmer (see the scheme) - is important as it is

\footnotetext{
* The measurements were performed with the friendly help of Dr. Gerold Endert, Dept. "Phospholipids".
} 
assumed to be stable in lipid bilayers [4,9]. In X-ray diffraction studies, the inner and the outer diameter of the channel were determined as approximately $5 \AA$ and $15 \AA$, respectively; the total length as $\sim 30 \AA$ [6]. (Different studies show, however, slightly varying channel dimensions.)

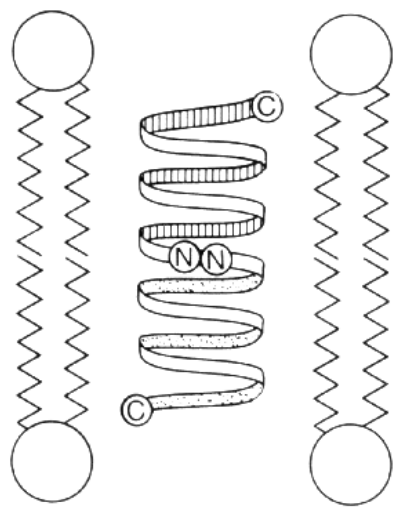

All hydrophobic side chains are on the outside of the helix, and hydrophilic peptide backbone carbonyls line the pore. Because of its availability, gramicidin A has frequently been used as a „model membrane protein“ for studying the perturbing influence of membrane proteins on lipids. Because of its lipid structure modulating activity, the peptide induces membrane fusion, causes packing defects in membranes and enhances lipid transbilayer movement [9].

\section{Alamethicin}

Alamethicin is a 20-residue peptide antibiotic produced by the fungus Trichoderma viride, that forms voltage-gated channels in membranes [5]. The alamethicin sequence given below includes unusual residues, $\alpha$-aminobutyric acid (Aib) and L-phenylalaninol (Phl).

Ac-Aib-Pro-Aib-Ala-Gln-Aib-Val-Aib-

\section{Gly-Leu-Aib-Pro-Val-Aib-Aib-Glu-Glu-Phl}

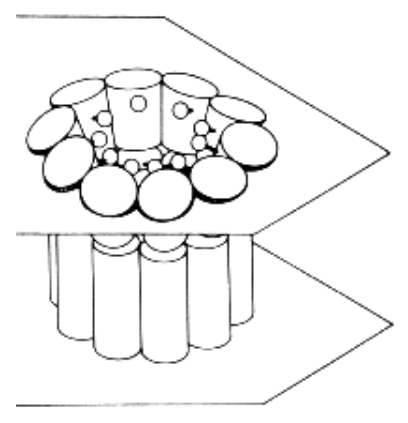

The dependence of the conductance of planar bilayers modified by alamethicin on the peptide concentration suggests that each channel contains at least 6-11 molecules. The channel length is about $32 \AA$ [6], which is sufficient to extend across the nonpolar parts of the bilayer. The cartoon on the left presents a schematic structure of the alamethicin pore, as modeled by [5]. 


\section{Lipid - Peptide Mixtures}

Gramicidin A was obtained as powder from Sigma (St. Luis, MO). Alamethicin (as powder) was provided by ICN Biochemicals Inc. (Aurora, $\mathrm{OH}$ ).

For preparing lipid-peptide mixtures, appropriate amounts of both substances were weighted and dissolved in a 2:1 chloroform/methanol mixture [9]. The solvent was evaporated by nitrogen gas flow. To ensure complete removal of the solvent the sample was dried under vacuum overnight. The mixed powder was then resuspended in buffer, and the desired vesicle dispersion was prepared as described above.

Samples required for the Atomic Force Microscopy measurements were prepared as follows [14]: Mixed lipid-peptide multilamellar vesicles were sonicated as described above to form small unilamellar vesicles. A small droplet of the vesicle solution was applied to a freshly cleaved mica surface at room temperature. Since the small unilamellar vesicles are not stable, they fuse and form a flat bilayer segment on the mica surface. Then, the excess vesicles were washed away from the membrane surface using a moderate salt solution like $150 \mathrm{mM} \mathrm{NaCl}$. The latter is said to be very effective in removing excess vesicles [14]. With non-charged membranes (like DPPC and DMPC), however, no significant difference in effectiveness was noticed between the use of buffer, water or salt solution. The AFM experiments were performed in water.

\section{References}

[1] Birge, N.O. and Nagel, S.R. (1985), Physical Review Letters, Vol. 54, 2674-2676

[2] Birge, N.O. and Nagel, S.R. (1987), Review of Scientific Instruments, Vol. 58, 14641470

[3] Ebel, H.F. (1999). Graduate thesis, Georg-August-Universität, Göttingen. Kalorische und strukturelle Umwandlungen von Lipid-Membranen: Kopplung von Wärmekapazität und Volumenexpansionskoeffizienten.

[4] Finkelstein, A. and Andersen, O.S. (1981), Journal of Membrane Biology, Vol. 59, $155-171$ 
[5] Fox, R.O. and Richards, F.M. (1982), Nature, Vol. 300, 325-330

[6] Gennis, R.B. (1989). Biomembranes: molecular structure and function, pp. 128-129, New York: Springer

[7] Grabitz, P. (2000). Graduate thesis, Georg-August-Universität, Göttingen. This work is in preparation and will be submitted at the end of 2000.

[8] Heimburg, T. (1998). Biochimica et Biophysica Acta, Vol. 1415, 147

[9] Killian, J.A. (1992), Biochimica et Biophysica Acta, Vol. 1113, 391-425

[10] Leyser, H. (1994). Graduate thesis, Technische Universität, München. Spektroskopie der frequenzabhängigen spezifischen Wärme an unterkühlten wässrigen Lösungen.

[11] Mabrey, S. and Sturtevant, J.M. (1978). High-sensitivity differential scanning calorimetry in the study of biomembranes and related model systems. In E. Korn (Ed.), Methods in Membrane Biology. Vol. 9 (237-274). New York: Plenum Press

[12] Plotnikov, V.V., Brandts, J.M., Lin, L.-N., and Brandts, J.F. (1997), Analytical Biochemistry, Vol. 250, 237-244

[13] Settles, M., Post, F., Müller, D., Schulte, A., and Doster, W. (1992), Biophysical Chemistry, Vol. 43, 107-116

[14] Shao, Z. and Yang, J. (1995), Quarterly Reviews of Biophysics, Vol. 28 (2), 195-251 


\section{Ising model of the lipid membrane and Monte Carlo simulations}

\subsection{Introduction}

Besides the use of mean field theories for describing lipid melting $[29,18,36]$ a common approach consists of the application of statistical thermodynamics models [30]. These models usually reduce the wealth of states of individual lipids into subensembles with given average energies and entropies. The distribution of states is then explored with Monte Carlo (MC) simulations. The advantage of these models over mean-field approaches is that they provide insight into the magnitude of enthalpy or volume fluctuations [19], and into domain formation $[32,1,5]$ within the lipid membrane plane. The obvious disadvantage is that the Monte Carlo like simulations used to evaluate the models produce no analytical solutions and are partially time-consuming.

A widely used lattice model is the Pink's multi-state model of the chain-melting phase transition [35,4]. This model is formulated in terms of ten conformational states, of which one is the fully ordered all-trans conformation and one is a highly excited liquid crystalline state. The eight remaining states are intermediate chain states which may be viewed as lowenergy excitation of the all-trans state. These eight states and the all-trans state are characteristic of the gel phase. In the Pink model one considers van der Waals interactions as well as membrane lateral pressure and interfacial energy. This description therefore requires a number of parameters and makes some important predictions on the molecular level $[33,31]$. However, the physics that lead to domain formation and that is necessary to rationalize the heat capacity profiles is already contained in the much simpler two-state model with only gel and fluid states, also referred as a Doniach-model [7]. It was shown that this model is able to describe the lipid phase transition adequately [38,39,20,21], using one single interfacial energy term, which is the only parameter required for the calculation that is not directly given by the experiment.

The main motivation for employing Monte Carlo simulations is that in complex systems the degeneracy of isoenergetic states cannot be calculated analytically and hence has to be explored numerically. A more efficient way for using the information from Monte Carlo 
simulations presents the histogram method [8]. Within this approach, the degeneracy of states is sampled instead of time averages during the simulation. The degeneracy does not depend on the parameters of the simulation and thus can be used to calculate quasianalytically mean values of various physical observables. This concept was widely applied to reduce computer time, for example to describe transitions in two-dimensional systems. One problem with this kind of analysis is that usually in a single Monte Carlo simulation only a small part of the phase space is explored. This limitation of the histogram method however can be overcome by combining histograms produced by simulations, which were performed at different sets of parameters [9]. Another way is to sample histograms close to the critical point where the fluctuations are especially large [22].

In this chapter, we describe in some detail the two-state model adopted for the lipid melting transition and the MC simulations performed. The matching procedure used to combine histograms produced by simulations at different sets of parameter is explained. Further, the constructed broad histogram was used to fit experimental heat capacity profiles. The heterogeneities in the membrane plane resulting from the large thermal fluctuations near the chain-melting transition are also discussed.

\subsection{Two-state model for the gel-to-fluid transition}

The lipid transition shell be described assuming that only two states, gel and fluid, are available for each individual lipid molecule. The lipids form a triangular lattice in which each molecule is surrounded by six nearest neighbors, that is, the coordination number is $z=6$.
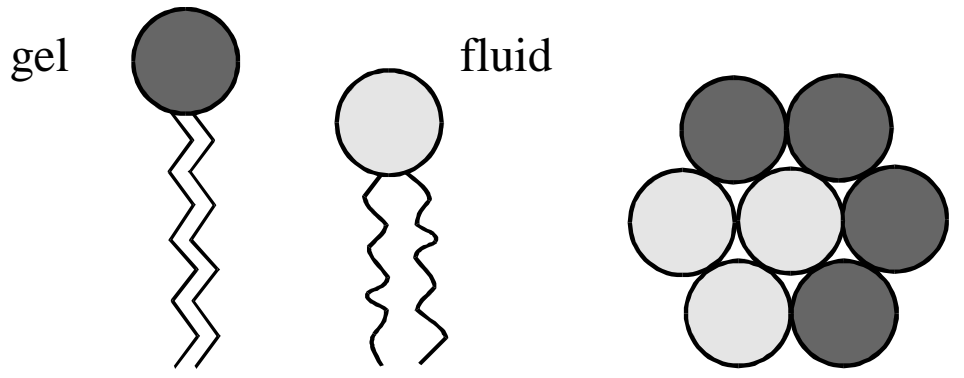

In our computer model the lipid monolayer is represented by a $31 \times 31$ square matrix of $n=961$ elements. Each matrix element refers to a lattice site (lipid molecule) of the monolayer. In order to minimize the edge effects, periodic boundary conditions have been 
utilized. Thus the lipid matrix corresponds to a torus (or donut) instead of a sphere. Since the model membrane systems are mostly vesicles (spheres), spherical boundary conditions [16] would be more appropriate. In this case, the two-dimensional membrane would be embedded in the surface of a sphere without introducing any physical boundaries. When spherical boundary conditions are applied, however, one must use a continuum model of the membrane because, in general, regular lattices (with a given coordination number) cannot be defined on the surface of a sphere.

Lipid-lipid interactions are taken into account through a nearest neighbor interaction free energy, $\varepsilon$. The free energy of each individual lipid molecule consists of two components, which are the intrinsic free energy $G_{i}$ (the index $i$ stands for $g$ (gel) or $f$ (fluid)) of the chain configuration and the sum over the nearest neighbor interaction free energies $\varepsilon_{i j}$. As long as the half-width of the gel-to-fluid transition is small, it is a reasonable simplification to assume that the nearest neighbor interactions $\varepsilon_{i j}$ are purely enthalpic [21], i.e. temperature independent. Then the total free energy of the lipid matrix is the sum over all $n$ lipids:

$$
G=n_{g} \cdot G_{g}+n_{f} \cdot G_{f}+n_{g g} \cdot \varepsilon_{g g}+n_{f f} \cdot \varepsilon_{f f}+n_{g f} \cdot \varepsilon_{g f}
$$

where $n_{i}$ is the number of lipids in state $i$ and $n_{i j}$ is the number of nearest neighbor interactions of lipids in states $i$ and $j$.

In the case of periodic boundary conditions the following two relationships exist between $n_{i}$ and $n_{i j}$ values for any matrix configuration:

$$
\begin{aligned}
& n_{f f}=\left(z \cdot n_{f}-n_{g f}\right) / 2 \\
& n_{g g}=\left(z \cdot n_{g}-n_{g f}\right) / 2
\end{aligned}
$$

where $z(=6)$ is the coordination number. Then the free energy of the system can be rewritten as

$$
G=n \cdot G_{g}+n_{f} \cdot(\Delta H-T \cdot \Delta S)+n_{g f} \cdot \omega_{g f}
$$

where $\Delta H=\left(H_{f}+z \cdot \varepsilon_{f f} / 2\right)-\left(H_{g}+z \cdot \varepsilon_{g g} / 2\right), \Delta S=\left(S_{f}-S_{g}\right)$ and $\omega_{g f}=\varepsilon_{g f}-\left(\varepsilon_{g g}+\varepsilon_{f f}\right) / 2$. The system reaches its energy minimum when all the lipids are in the gel state. Then the excess free energy of the lipid system is given by

$$
\Delta G=G-n \cdot G_{g}=n_{f} \cdot(\Delta H-T \cdot \Delta S)+n_{g f} \cdot \omega_{g f}
$$


Formally, the model described in Equation (2.4) resembles an Ising model in a field, where $(\Delta H-T \cdot \Delta S)$ acts as the temperature dependent field. Therefore, we will refer further to the model as to a two-state Ising model.

The magnitude of $\omega_{g f}$ defines the cooperativity of the transition. The critical point for an infinitely large triangular lattice is given by [10]

$$
\frac{k_{B} \cdot N_{A} \cdot T_{c}}{z \cdot\left(\omega_{c} / 2\right)}=0.6068256
$$

where $T_{c}$ is the critical temperature, $\omega_{c}$ is the critical cooperativity (in $[\mathrm{cal} / \mathrm{mol}]$ ) and $z(=6)$ is the coordination number. If we choose $T_{c}=T_{m}=310.3 \mathrm{~K}$, which is the melting temperature in the case of small unilamellar vesicles of DPPC, as the (bulk) critical value of the cooperativity parameter we obtain $\omega_{c}=338 \mathrm{cal} / \mathrm{mol}$.

When $\omega_{g f}=0$, the transition is non-cooperative; each lipid melts independently, producing a very broad transition. If $\omega_{g f} \geq \omega_{c}$, the melting is an all-or-none transition.

In a two-state model, the excess free energy $\Delta G=\Delta H-T_{m} \cdot \Delta S$ is equal to zero at the transition mid-point, $T_{m}$, and hence $\Delta S=\Delta H / T_{m}$ [7]. This means that in our two-state model we have in fact three parameters to determine - the enthalpy $\Delta H$, the transition temperature $T_{m}$ and the cooperativity parameter $\omega_{g f}$. The enthalpy $\Delta H$ and the mid-point of the gel-to-fluid transition $T_{m}$ can be determined directly from a calorimetric experiment the enthalpy is the integral of the complete heat capacity profile over the temperature, and the transition temperature is the position of the heat capacity maximum. The cooperativity parameter $\omega_{g f}$ is obtained by a comparison of the simulated results with the experimental transition half width. In $[38,39]$ it was shown that the qualitative and the quantitative behavior of the heat capacity profile of DPPC small unilamellar vesicles (SUVs) can be described in that simple way. 


\subsection{Fluctuations and heat capacity}

By applying Monte Carlo methods one can generate thermal fluctuations of the lipid monolayer. Then, using the fluctuation-dissipation theorem from statistical mechanics [24]

$$
C_{p}=\left(\frac{\partial\langle H\rangle}{\partial T}\right)_{p}=\frac{\left\langle H^{2}\right\rangle-\langle H\rangle^{2}}{R T^{2}}
$$

one calculates numerically the heat capacity of the system. The mean values $\left\langle H^{2}\right\rangle$ and $\langle H\rangle$ are averaged over the time (i.e. over many MC cycles). An MC cycle simulates the thermal fluctuations of the monolayer within a very short time interval. The points within the lattice are picked at random and then its state is switched according to the statistical mechanical probability of such a change. We apply here a standard Galuber algorithm [12] whose elementary steps are briefly described as follows:

- Pick at random a lattice point.

- Change its state.

- Calculate the Gibbs energy difference between the new and the old matrix configuration

$$
\delta G= \pm(\Delta H-T \cdot \Delta S)+\Delta n_{g f} \cdot \omega_{g f}
$$

where $+/$ - is for change gel-fluid/fluid-gel and $\Delta n_{g f}$ is the increase of the unlike nearest neighbor contacts.

- Calculate the probability for the change

$$
P=\frac{K(T)}{1+K(T)}, \quad \text { with statistical weights } \quad K(T)=\exp \left(-\frac{\delta G}{R T}\right)
$$

- Generate a random number RAN and compare it with the calculated probability.

- Make a decision (YES or NO) for the change.

$$
\begin{array}{lll}
R A N \leq P & \mapsto & Y E S \\
R A N>P & \mapsto & N O
\end{array}
$$

The scheme below illustrates the change in lipid state during a single MC step. The gelstate and the fluid-state lipids are depicted by dark and light gray, respectively. 


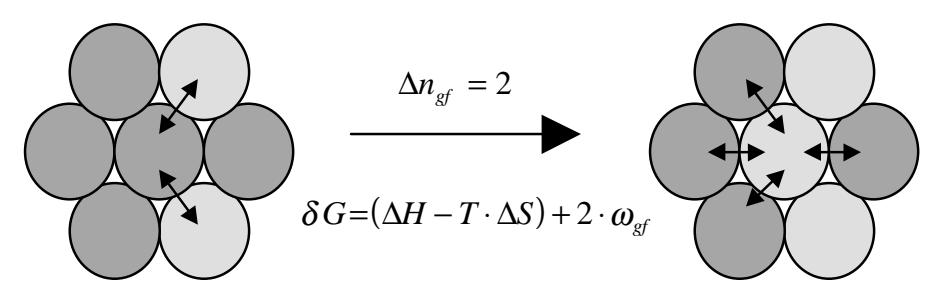

A representative MC-snapshot of the lipid matrix is shown in Figure 2.1.

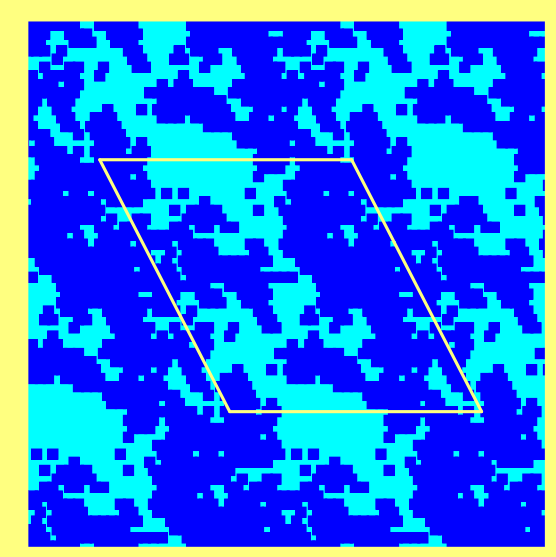

Figure 2.1. Typical Monte Carlo snapshot of a lipid matrix at the melting point for $\omega_{g f}=300 \mathrm{cal} / \mathrm{mol}$. Dark dots represent gel lipids, light gray dots represent fluid lipids. A 31.31 matrix with periodic boundary conditions is shown. The unit cell of the simulation is indicated by the rhombic box in the center of the picture.

It is evident that at this value of the cooperativity parameter the correlation length may become very large and even comparable with the size of the computer matrix. Thus, finitesize effects must be accounted. Nevertheless, it is important to note that a large correlation length does not necessarily imply that the system is close to a critical point. Our results are based on calculations on a 31.31 lattice. Calculations on a larger lattice 61.61 for $\omega_{g f} \leq 325 \mathrm{cal} / \mathrm{mol}$ were found to give the same results within statistical accuracy, thus demonstrating that finite-size effects in this regime are negligible.

The Monte Carlo steps are repeated many times and, for each matrix configuration generated, the enthalpy is computed: $H=n_{f} \cdot(\Delta H-T \cdot \Delta S)+n_{g f} \cdot \omega_{g f}$. Then the average values $\langle H\rangle$ and $\left\langle H^{2}\right\rangle$ are calculated in order to determine the heat capacity at given 
temperature. Figure 2.2 demonstrates the significant effect of the cooperativity parameter on the lipid melting transition.

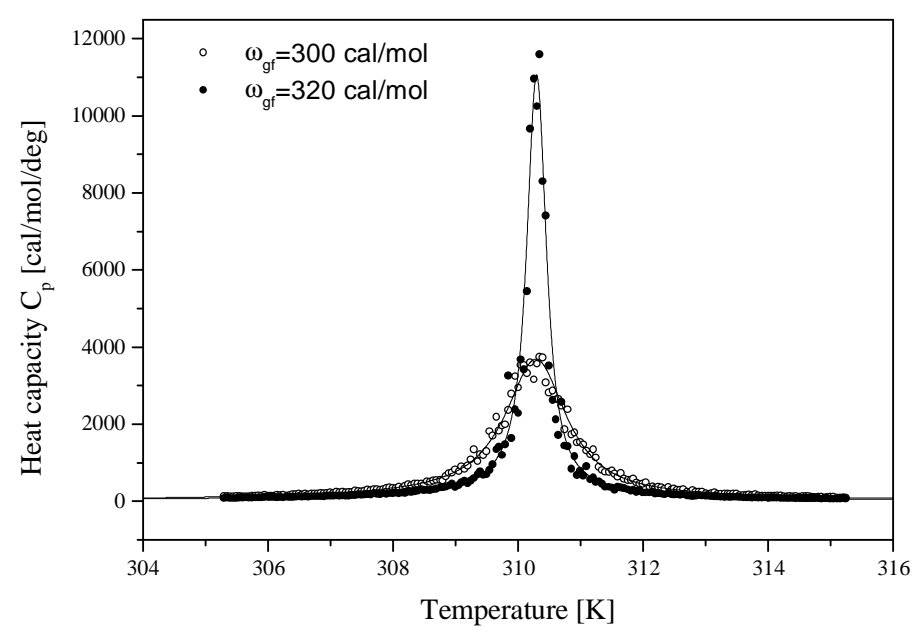

Figure 2.2. Calculated heat capacity profiles of a pure lipid membrane at two different values for the cooperativity parameter $\omega_{g f}\left(\omega_{g f}=300 \mathrm{cal} / \mathrm{mol}\right.$ and $\left.\omega_{g f}=320 \mathrm{cal} / \mathrm{mol}\right), \quad \Delta H=8700 \mathrm{cal} / \mathrm{mol}, \quad$ and $T_{m}=310.3 \mathrm{~K}$. Circles: Heat capacities obtained directly from a MC simulation. Open circles: $\omega_{g f}=300 \mathrm{cal} / \mathrm{mol}$; Solid circles: $\omega_{g f}=320 \mathrm{cal} / \mathrm{mol}$. Lines: Respective calculations, using the histogram method (Section 2.4). The calculations were performed using a 31.31 matrix with periodic boundary conditions.

It is evident that increasing the interfacial free energy parameter increases the cooperativity of the transition, resulting in both a decreasing transition half-width and an increasing heat capacity maximum. Even small changes in $\omega_{g f}$ influence drastically the phase transition in the demonstrated case only $6.7 \%$ increase of the cooperativity parameter decreases the transition half width more than 3 times and increases the heat capacity maximum about 3 times.

This example demonstrates also the disadvantage of the applied method, that means that it is very time-consuming. One needs to run a long simulation for each $T$ in order to be able to calculate accurately the mean values $\left\langle H^{2}\right\rangle$ and $\langle H\rangle$. Since $\omega_{g f}$ is an unknown parameter in the model and has to be obtained by comparison between the experimental heat capacity profile and the simulated one, the procedure described above is not very efficient. 


\subsection{Histogram method for MC data analysis}

A more efficient approach for analysis of the MC data was proposed by [8]. The method makes use of the fact that for long simulation times the mean values obtained by time and by ensemble averaging are equal for ergodic systems [18]. Our main aim is to calculate heat capacity profiles, in order to compare them with experiment. Therefore, we only consider physical observables which in a micro-state (matrix configuration) are expressed as functions of the number of fluid lipids, $n_{f}$, and the number of unlike nearest-neighbor contacts, $n_{g f}$. The latter are also the variables necessary to describe the Gibbs free energy of the lipid system within the framework of the two-sate Ising model (Equation (2.4)).

Thus, instead of calculating the mean value of a certain physical observable $\langle X\rangle$ over the time, we will average it over all matrix configurations at a fixed set of parameters $\zeta=\left(T, \Delta H, T_{m}, \omega_{g f}\right)$, using the statistical thermodynamic expression:

$$
\begin{aligned}
& \langle X(T)\rangle=\sum_{n_{f}} \sum_{n_{g f}} X\left(n_{f}, n_{g f}\right) \cdot P\left(\zeta, n_{f}, n_{g f}\right) \\
& =\frac{\sum_{n_{f}} \sum_{n_{g f}} X\left(n_{f}, n_{g f}\right) \cdot \Omega\left(n_{f}, n_{g f}\right) \cdot \exp \left(-\frac{n_{f} \cdot(\Delta H-T \cdot \Delta S)+n_{g f} \cdot \omega_{g f}}{R T}\right)}{\sum_{n_{f}} \sum_{n_{g f}} \Omega\left(n_{f}, n_{g f}\right) \cdot \exp \left(-\frac{n_{f} \cdot(\Delta H-T \cdot \Delta S)+n_{g f} \cdot \omega_{g f}}{R T}\right)}
\end{aligned}
$$

where $P\left(\zeta, n_{f}, n_{g f}\right)$ is the probability for a given set of parameters, $\zeta$, of finding a lipid matrix configuration with a number of lipids in the fluid state, $n_{f}$, and of gel-fluid contacts, $n_{g f}$, and $\Omega\left(n_{f}, n_{g f}\right)$ is the number of independent ways of generating such a configuration (i.e., the degeneracy). Now, the crucial point is that the degeneracy $\Omega\left(n_{f}, n_{g f}\right)$ does not depend on the set of parameters, $\zeta$, of the MC simulation. It is simply a function which gives information about the number of matrix configurations with $n_{f}$ fluid lipids and number $n_{g f}$ of the unlike nearest neighbor contacts. Thus, $\Omega\left(n_{f}, n_{g f}\right)$ is strongly dependent on the size of the computer matrix*. For large systems (in our case 31.31 elements) the degeneracy cannot be determined analytically. But instead of calculating

\footnotetext{
* The matrix size, as a parameter of the degeneracy function, will be omitted for clarity.
} 
average values of physical observables only, one can use the MC simulation to sample $\Omega\left(n_{f}, n_{g f}\right)$. Once $\Omega\left(n_{f}, n_{g f}\right)$ is known, the mean value of an observable $\langle X\rangle$ can be derived for any given set of parameters $\zeta$ by using Equation (2.7) above without performing a new MC simulation (!). This is the essence of the „histogram method of MC data analysis“ [8].

\subsection{Construction of a broad monolayer histogram}

Each MC simulation produces a probability distribution of states

$$
P\left(n_{f}, n_{g f}, \zeta\right)=\frac{\Omega\left(n_{f}, n_{g f}\right) \cdot \exp \left(-\Delta G\left(n_{f}, n_{g f}, \zeta\right) / R \cdot T\right)}{\sum_{n_{f}, n_{g f}} \Omega\left(n_{f}, n_{g f}\right) \cdot \exp \left(-\Delta G\left(n_{f}, n_{g f}, \zeta\right) / R \cdot T\right)}
$$

which can be rastered into bins, where each bin contains the sum of the probabilities in a segment of the phase space. Since in the two-dimensional model the distribution function $\Omega\left(n_{f}, n_{g f}\right)$ depends on two variables, $n_{f}$ and $n_{g f}$, the information of the probability distribution is put into a two-dimensional histogram with $100 \cdot 200$ bin. $\Delta f=\Delta \bar{n}_{g f}=0.01$ has been chosen as the size of a single bin, where, for simplicity, we use as variables the fraction of the fluid lipids, $f=n_{f} / n$, and the mean number of unlike near neighbor contacts per lipid, $\bar{n}_{g f}=n_{g f} / n$. The maximum value for $\bar{n}_{g f}$ is 2 , i.e., $0 \leq \bar{n}_{g f} \leq 2$. This maximum is reached when $f=0.5$ and the matrix places are occupied alternately with gel and fluid lipids. In practice, such a configuration is generated only when the transition is not cooperative at all, $\omega_{g f}=0$, and in systems of relatively small size. In our simulations, such a configuration was never generated on a $31 \cdot 31$ matrix.

The size of the bin determines the accuracy of the description of the degeneracy function. But a smaller bin-size leads to a large number of bins. More MC simulations are required and, accordingly, more CPU time, for exploring the whole phase space. Therefore, the decision concerning the bin size is a kind of compromise. However, we tested the accuracy of the raster by comparison between heat capacities calculated directly by running $\mathrm{MC}$ simulation (Equation 2.6) and determined from the collected histogram. 
A histogram obtained by running a MC simulation will be called „measured histogram“. The measured histogram may be used to determine the probability distribution at any other set of parameters $\zeta^{*}$ :

$$
P^{*}\left(n_{f}, n_{g f}, \zeta^{*}\right)=\frac{P\left(n_{f}, n_{g f}, \zeta\right) \cdot \exp \left(\frac{\Delta G\left(n_{f}, n_{g f}, \zeta\right)}{R T}-\frac{\Delta G\left(n_{f}, n_{g f}, \zeta^{*}\right)}{R T^{*}}\right)}{\sum_{n_{f}} \sum_{n_{g f}} P\left(n_{f}, n_{g f}, \zeta\right) \cdot \exp \left(\frac{\Delta G\left(n_{f}, n_{g f}, \zeta\right)}{R T}-\frac{\Delta G\left(n_{f}, n_{g f}, \zeta^{*}\right)}{R T^{*}}\right)}
$$

It is evident that the probability distribution $P^{*}\left(n_{f}, n_{g f}, \zeta^{*}\right)$ can only be used to calculate thermodynamic functions if the measured histogram, $P\left(n_{f}, n_{g f}, \zeta\right)$, covers most of the available (!) phase space. Because of the finite Monte Carlo sampling it is impossible to cover the whole configuration space $\left(n_{f}, n_{g f}\right)$ by a single histogram. Figure 2.3 shows a representative probability distribution of states for a monolayer at the heat capacity maximum.

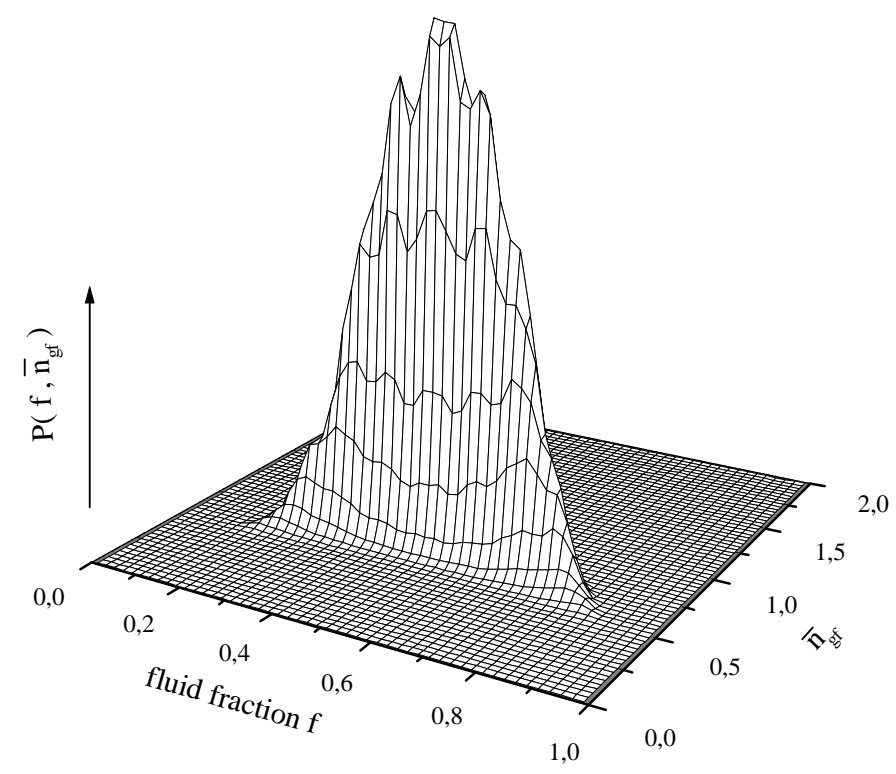

Figure 2.3. Histogram of the distribution of states of a monolayer at the mid-point of the gel-to-fluid transition, $T=T_{m}$, for $\omega_{g f}=310 \mathrm{cal} / \mathrm{mol}$. The maximum is at $f=0.5$. 
The statistics of MC-sampling is good, close to the distribution maximum. However, this is not the case in the outer wings of the distribution since there are only a small number of events generated. Thus, the size of the original histogram limits the range to which Equation (2.9) is applicable. The accuracy of this procedure, however, can be significantly improved when histograms obtained for various sets of parameters, $\zeta^{*}$, are merged into one large histogram $[9,3]$. In the present work this is done in the following manner:

Let us call the normalized histogram $P\left(n_{f}, n_{g f}, \zeta\right)$ obtained at a set of parameters $\zeta$, primary histogram, and the normalized histogram $P^{*}\left(n_{f}, n_{g f}, \zeta^{*}\right)$ measured at parameters $\zeta^{*}$, secondary histogram. For the matching procedure it is necessary that the two histograms have a good overlap.

The part of the overlapping region, $M^{2 d}$, where the secondary distribution has better statistics than the primary one, is defined as the part of the phase space where

$$
P^{*}\left(n_{f}, n_{g f}, \zeta^{*}\right)>P\left(n_{f}, n_{g f}, \zeta\right) \quad \text { for }\left\{n_{f}, n_{g f}\right\} \in M^{2 d}
$$

The secondary distribution is then recalculated (Equation 2.9) using the parameters of the primary one, $\zeta^{*} \rightarrow \zeta$.

$$
P^{*}\left(n_{f}, n_{g f}, \zeta^{*}\right) \rightarrow \widetilde{P}^{*}\left(n_{f}, n_{g f}, \zeta\right)
$$

According to the Equation (2.9), the recalculated histogram is also normalized. A scaling ratio is determined in the following way:

$$
r=\frac{\sum_{\left\{n_{f}, n_{g f}\right\} \in M^{2 d}} P\left(n_{f}, n_{g f}, \zeta\right)}{\sum_{\left\{n_{f}, n_{g f}\right\}} \widetilde{P}^{*}\left(n_{f}, n_{g f}, \zeta\right)}
$$

Then the primary histogram is improved and extended as follows:

1) if $\left\{n_{f}, n_{g f}\right\} \in M^{2 d}$, then $P\left(n_{f}, n_{g f}, \zeta\right)=r \cdot \widetilde{P}^{*}\left(n_{f}, n_{g f}, \zeta\right)$

2) if $\left\{n_{f}, n_{g f}\right\} \notin M^{2 d}$ and 
2.1) if $P^{*}\left(n_{f}, n_{g f}, \zeta^{*}\right)>P\left(n_{f}, n_{g f}, \zeta\right)$, then $P\left(n_{f}, n_{g f}, \zeta\right)=r \cdot \widetilde{P}^{*}\left(n_{f}, n_{g f}, \zeta\right)$

2.2) if $P^{*}\left(n_{f}, n_{g f}, \zeta^{*}\right)<P\left(n_{f}, n_{g f}, \zeta\right)$, then $P\left(n_{f}, n_{g f}, \zeta\right)$ does not change.

A normalization of the extended histogram follows.

Step 1) here implies that the tails of the primary histogram are replaced with more accurate parts from the secondary histogram. Further, the accurate rest of the secondary histogram is used to extend the primary distribution even outside the overlapping region - step 2.1. This latter step differs from the matching method proposed in [3] where only 1) and 2.2) were applied.

The matching method, presented here, is very similar to the „multistage sampling“ technique of Valleau and Card [40], who considered overlapping energy distributions in the calculation of the free energy of the system of hard spheres with Coulombic forces.

The combination of many histograms into one is shown in Figure 2.4 (left hand panel) for 12 histograms (obtained for a fixed value for $\omega_{g f}$ at different temperatures) and for 84 histograms (obtained for a general set of different temperatures, $T$, and cooperativity parameters, $\left.\omega_{g f}\right)$ in Figure 2.4 (right hand panel). The outer limits of the histograms show the part of the phase space explored by the respective MC simulation. As will be demonstrated below, the broad histogram constructed in that way covers all the relevant phase space necessary to describe the melting transition in lipid dispersions.

The broad histogram was obtained combining the distributions sampled at the following parameters: $\Delta H=8700 \mathrm{cal} / \mathrm{mol}, T_{m}=310.3 \mathrm{~K}, \omega_{g f} \in\{260,270,280,290,300,310,325 \mathrm{cal} / \mathrm{mol}\}$ and $T \in\left\{T_{m}, T_{m} \pm 0.5, T_{m} \pm 15, T_{m} \pm 2.5, T_{m} \pm 3.5, T_{m} \pm 45, T_{m} \pm 5.5\right\}$. At the melting temperature the histograms were sampled over $3 \cdot 10^{6} \mathrm{MC}$ cycles and in the remaining cases - over $10^{6}$ $\mathrm{MC}$ cycles (each MC cycle is a complete random walk through the whole matrix, i.e., each lipid in the matrix was picked once). 

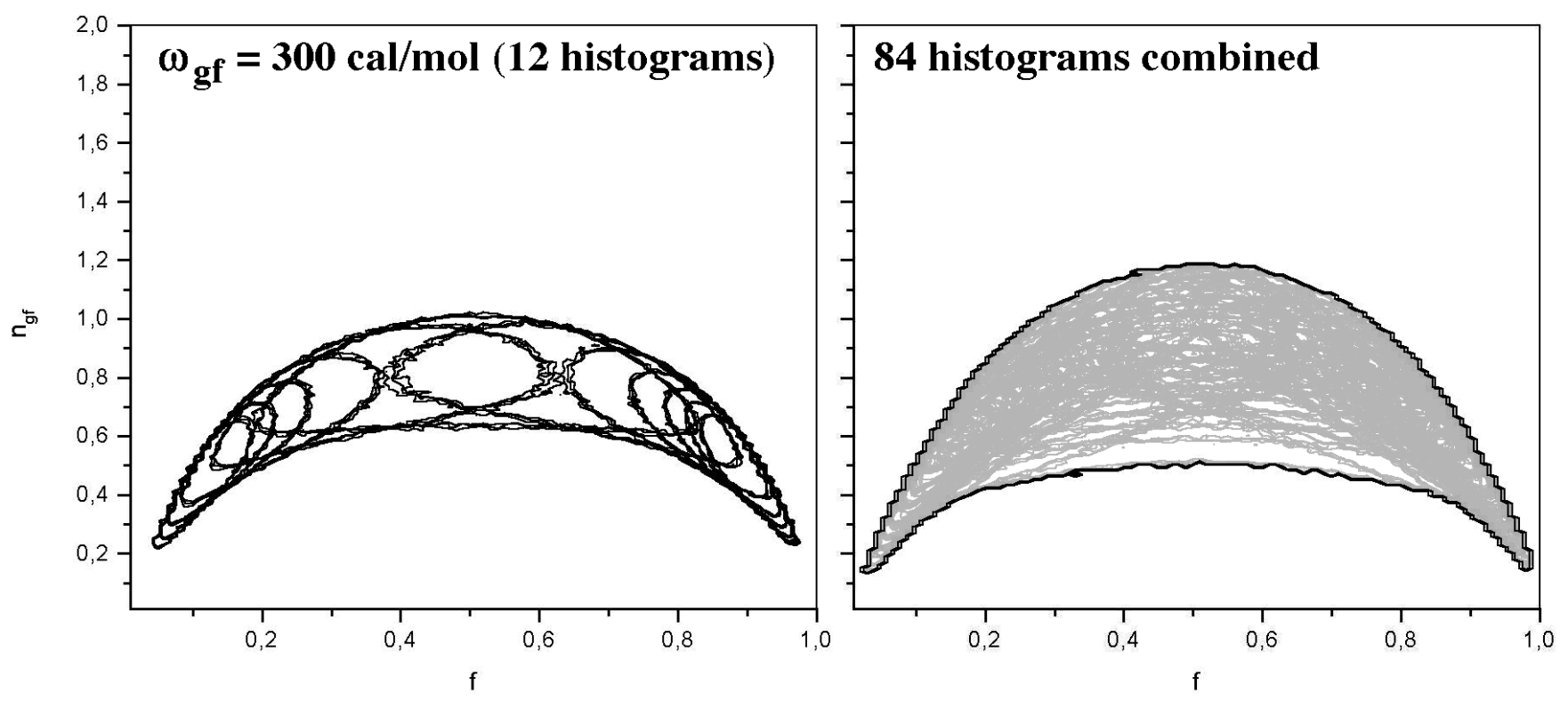

Figure 2.4. Top view of histograms, used for construction of the broad histogram. Left: Histograms at various temperatures, $\omega_{g f}=300 \mathrm{cal} / \mathrm{mol}$. Right: All measured distributions used for the construction of the broad histogram (gray lines). The lines enclose the parts of the phase space explored by the respective MC simulations. Only events are accounted which occur with probability $>10^{-5}$. Note the good overlap between neighboring histograms (on the left). It is essential for the success of the matching procedure.

From this general histogram the individual histograms for each fixed set of parameters, $\zeta$, can be obtained. Figure 2.5 shows the effect of the temperature changes close to the melting point (fixed $\omega_{g f}$ ) on the histograms. The distribution maximum moves to larger values of $n_{f}$ with increasing temperature because lipids are melting.

Histograms obtained at the heat capacity maximum, but with different values for the cooperativity parameter, $\omega_{g f}$, are presented in Figure 2.6. Upon increase in $\omega_{g f}$ the histogram undergoes a change from a profile with Gaussian cross-section to a histogram with two maxima. While the upper histogram indicates that the transition is continuous, the lower one corresponds to a first order like behavior where two membrane states may coexist [28]. The center panel of Figure 2.6 shows a monolayer close to its critical point. 


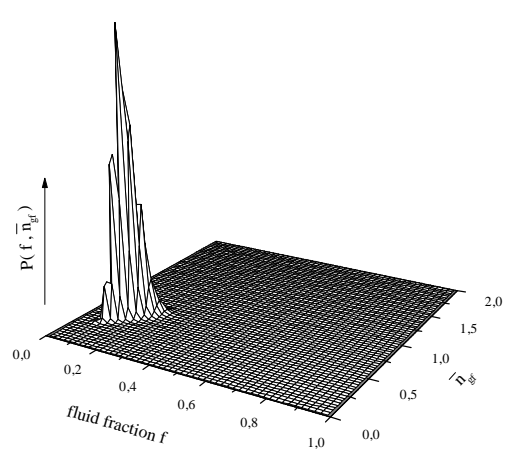

$T_{m}-2$
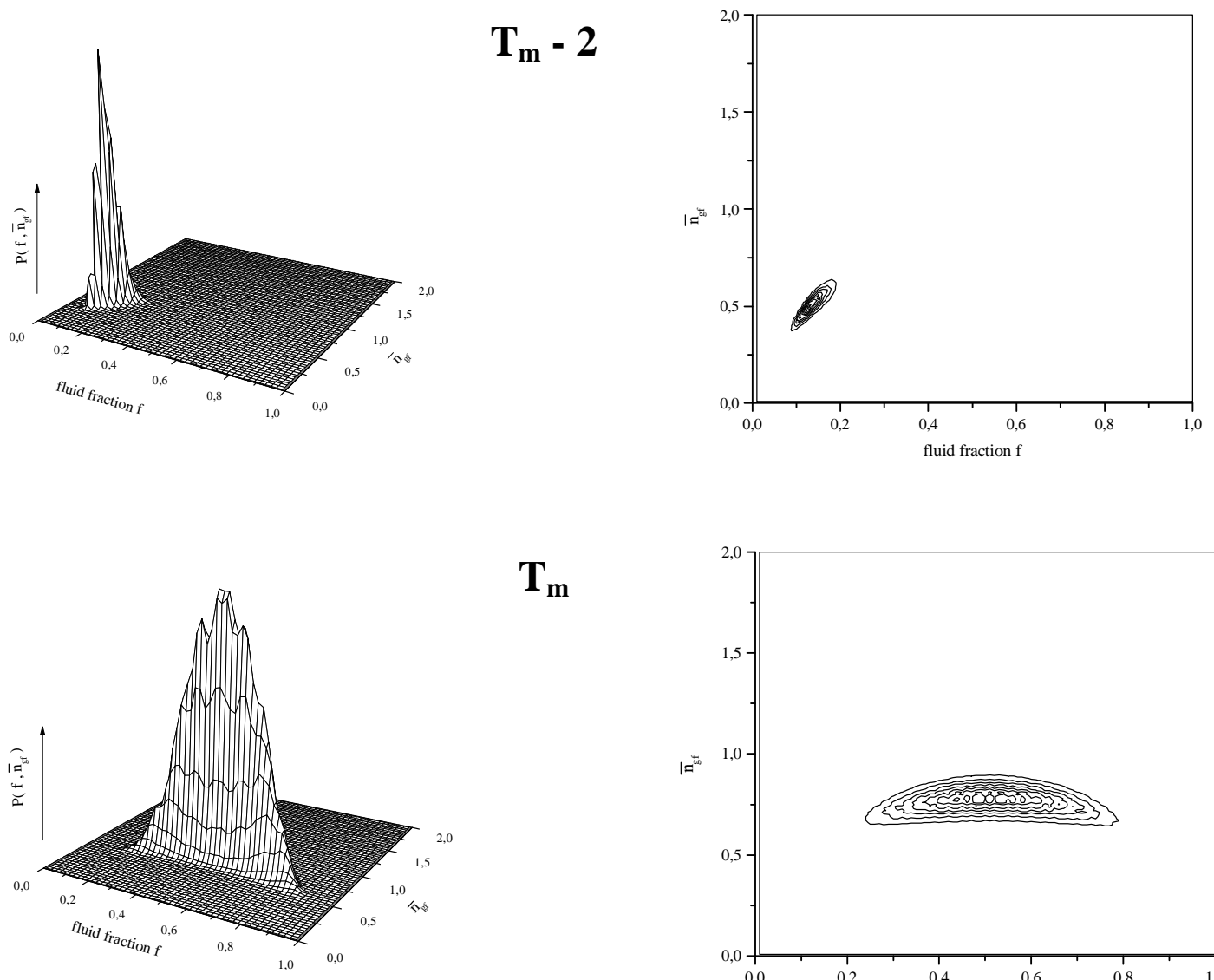

$\mathbf{T}_{\mathbf{m}}$

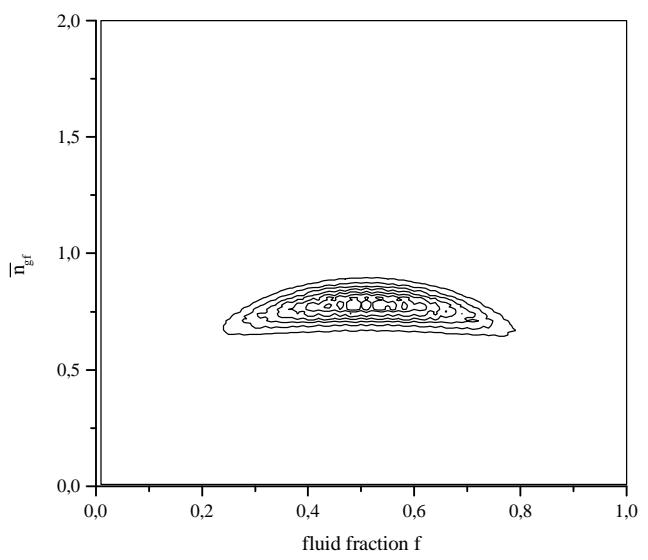

$\mathbf{T}_{\mathrm{m}}+\mathbf{2}$
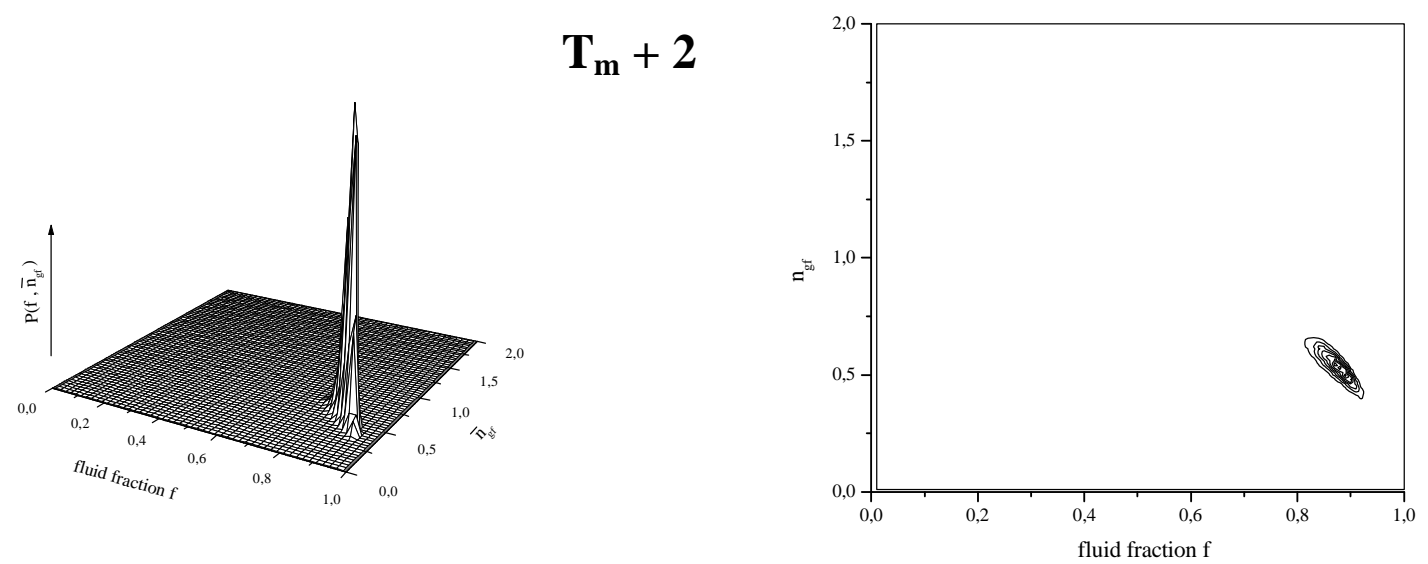

Figure 2.5. Surface (left) and contour (right) plot of the probability distributions of states for $\omega_{g f}=310 \mathrm{cal} / \mathrm{mol}$. Top: below $T_{m}$; Center: at $T_{m}$; Bottom: above $T_{m}$. 

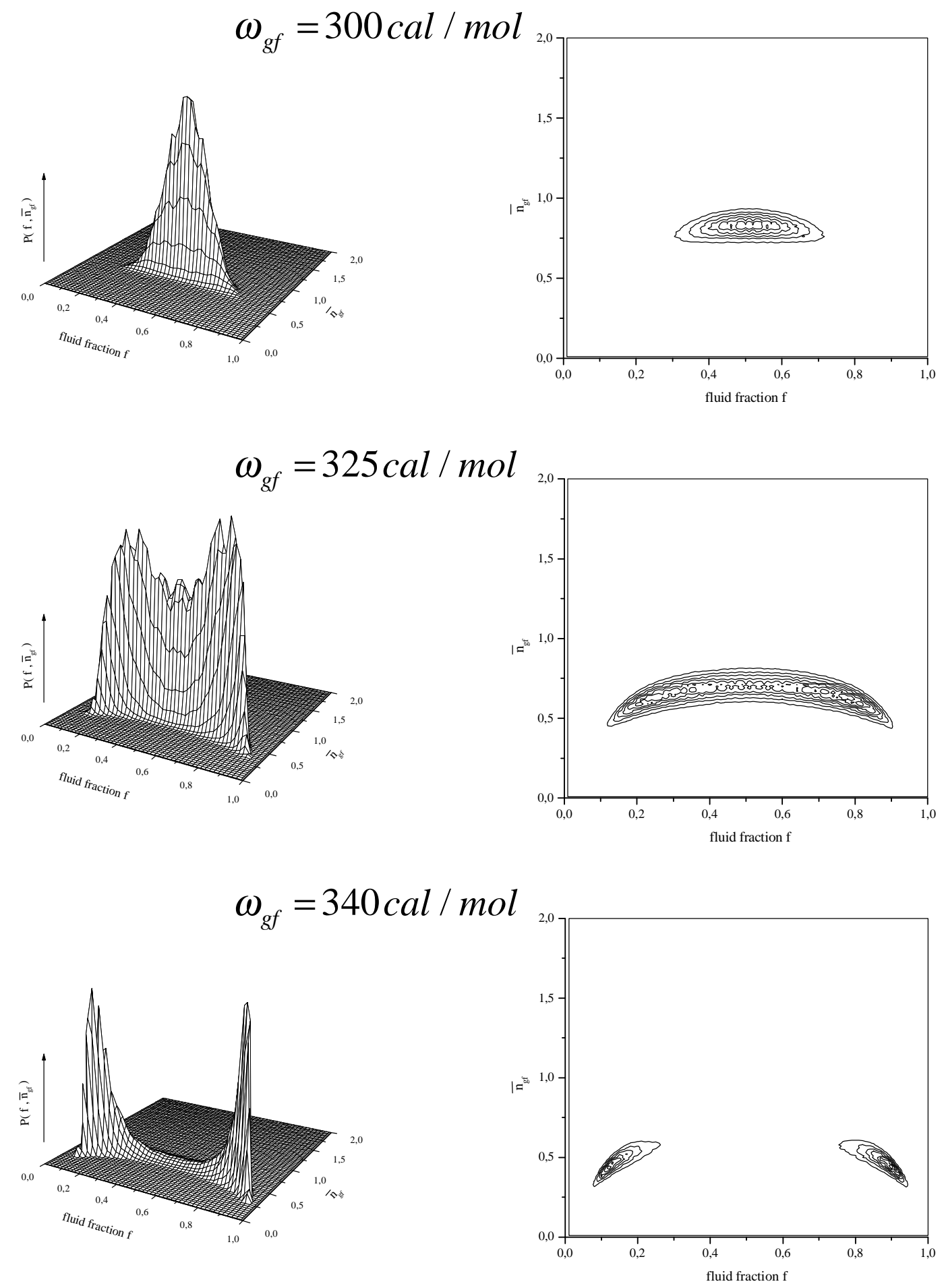

Figure 2.6. Histograms of the distribution of states at different cooperativities $\left(\omega_{g f}=300 \mathrm{cal} / \mathrm{mol}, \omega_{g f}=325 \mathrm{cal} / \mathrm{mol}\right.$ and $\left.\omega_{g f}=340 \mathrm{cal} / \mathrm{mol}\right)$ at the melting temperature, $T_{m}$. The top histogram represents the non-first order transition, the center histogram corresponds to a situation close to the critical point and the bottom histogram shows a first order-like behavior. The histogram has been obtained from a MC simulation performed on a $31 \cdot 31$ matrix. 
It is important to note that since the degeneracy $\Omega\left(n_{f}, n_{g f}\right)$ depends on the system size, the calculated histograms are also dependent on the size of the computer matrix. The latter is of finite size which leads to a finite width of the maxima in the histogram. When the distribution of states displays two maxima, the heat capacity determined by the MC simulation becomes size-dependent [28]. According to [28], the simulated system undergoes a first-order phase transition when the barrier between the two maxima decreases with system size, for a second-order phase transition it remains constant and if there is no phase transition, the barrier increases with increasing system size.

\subsection{Fit of the measured heat capacity profiles}

The constructed broad histogram can be used to calculate heat capacity profiles quasianalytically ${ }^{\star}$ In Figure 2.2 (above) both heat capacity profiles calculated from the broad histogram and from a direct MC simulation are compared. The good agreement between the two kinds of calculations suggests that the broad histogram constructed in that way is accurate enough to be used for determination of heat capacity profiles without running a MC simulation for each set of parameters. In particular, the histogram method can be applied to fit experimental DSC profiles in order to determine the value of the cooperativity parameter.

Neglecting curvature effects, one may assume that the lipid bilayer consists of two uncoupled monolayers (meaning that each monolayer may explore the phase space without any correlation with the other monolayer). Under these conditions the monolayer histogram technique can be used to generate quasi-analytical fits for experimentally obtained heat capacity profiles (Figure 2.7). The three parameters entering Equation (2.9), $\left(\Delta H, T_{m}, \omega_{g f}\right)$, can be determined from the integrated heat capacity, the melting point and the transition half width. In Figure 2.7, this has been done for three different preparations of dipalmitoyl phosphatidylcholine (DPPC) vesicles. When dispersed in water, DPPC spontaneously forms multilamellar vesicles (MLV) in the size range of up to $1000 \mathrm{~nm}$ (Figure $2.7 \mathrm{left}$ ). These vesicles can be transformed into small unilamellar vesicles (SUV) of approximately

\footnotetext{
- Meaning that the histogram provides numbers for an analytical function for the heat capacity.
} 
$20 \mathrm{~nm}^{*}$ radius by ultrasonification (Figure 2.7 right hand side). Since SUVs are metastable, they slowly fuse into large unilamellar vesicles (LUVs) of a diameter of approximately 100 $\mathrm{nm}^{*}$ (Figure 2.7 - center). The three vesicle preparations display very different transition cooperativities. MLVs display a transition half-width of less than $0.1 \mathrm{deg}$, whereas LUVs show $1 \mathrm{deg}$ and SUVs 2-3 deg half-width. The second peak in the heat capacity profile of SUVs stems from LUVs formed by some spontaneous fusion events (c.f. center trace). Also shown in Figure 2.7 are fits to the curves using the histogram technique. The input parameters into the calculation are the experimental values for the melting enthalpy, $\Delta H$, and the transition temperature, $T_{m}$.
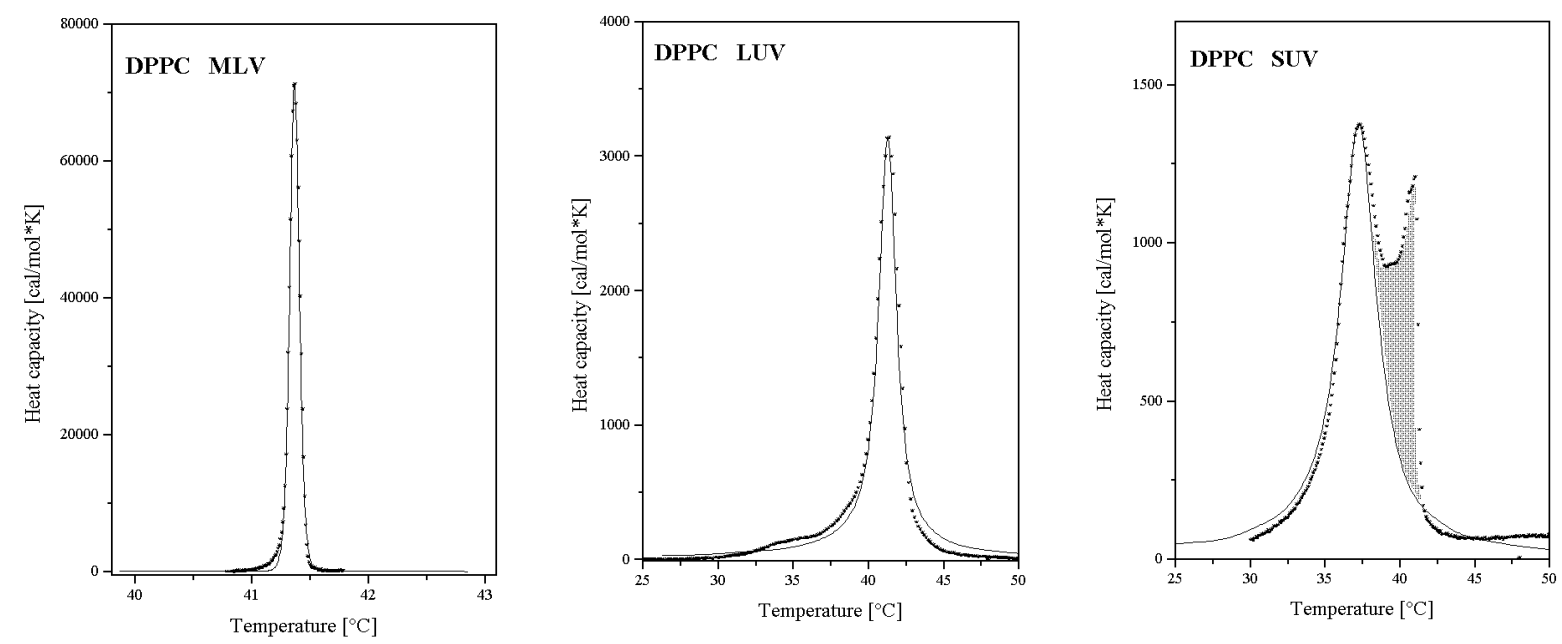

Figure 2.7. Fits of experimental heat capacity profiles of dipalmitoyl phosphatidylcholine (DPPC) vesicles from different preparations. Left: MLV, Center: LUV, Right: SUV. The shaded area represents a residual fraction of LUV in the SUV-preparation. Note the different scaling in the temperature axes. The MLV transition is very cooperative with a half-width of less then $0.1 \mathrm{deg}$. Fitting parameters are given in the text.

In spite of the fact that the three different vesicle preparations display heat capacity traces of different shapes, the enthalpies used for the fitting were the same $(\Delta H=8700 \mathrm{cal} / \mathrm{mol})$. To obtain calculated profiles similar to the experimental curves we mainly adjusted the

\footnotetext{
${ }^{*}$ See Figure 1.5 in Chapter 1.
} 
cooperative parameter, $\omega_{g f}$. For MLV we used $\omega_{g f}=377 \mathrm{cal} / \mathrm{mol}$, for LUV we used $297.5 \mathrm{cal} / \mathrm{mol}$ and for SUV $272 \mathrm{cal} / \mathrm{mol}$. It can be seen that the unlike nearest neighbor contributions are relatively small (about $0.5 \mathrm{kT}$ per interaction) and that relatively small changes in this parameter may significantly influence the shape of the heat capacity profiles. The value for MLV (Figure 2.7 - left hand side) corresponds to a first order like transition, whereas the values for LUV and SUV rather correspond to a continuous transition (compare to Figure 2.6). The latter supports the general notion that lipid melting transitions are generally close to the critical point.

The outer monolayer of the smallest vesicles (SUVs) contains about 2000 molecules, i.e. more than twice the number of lipid molecules in the simulated system. Thus, if finite-size effects are significant, how do the fit-values, $\omega_{g f}$, change with increasing system size? We mentioned already that for $\omega_{g f} \leq 325 \mathrm{cal} / \mathrm{mol}$, i.e., when the probability distribution of states displays only one maximum, no finite-size effects on the values of the calculated heat capacities are expected. In Figure 2.8 the fits calculated form the histogram (generated on a 31.31 matrix) are compared to heat capacity profiles calculated by running MC simulations for each temperature on a 61.61 matrix.
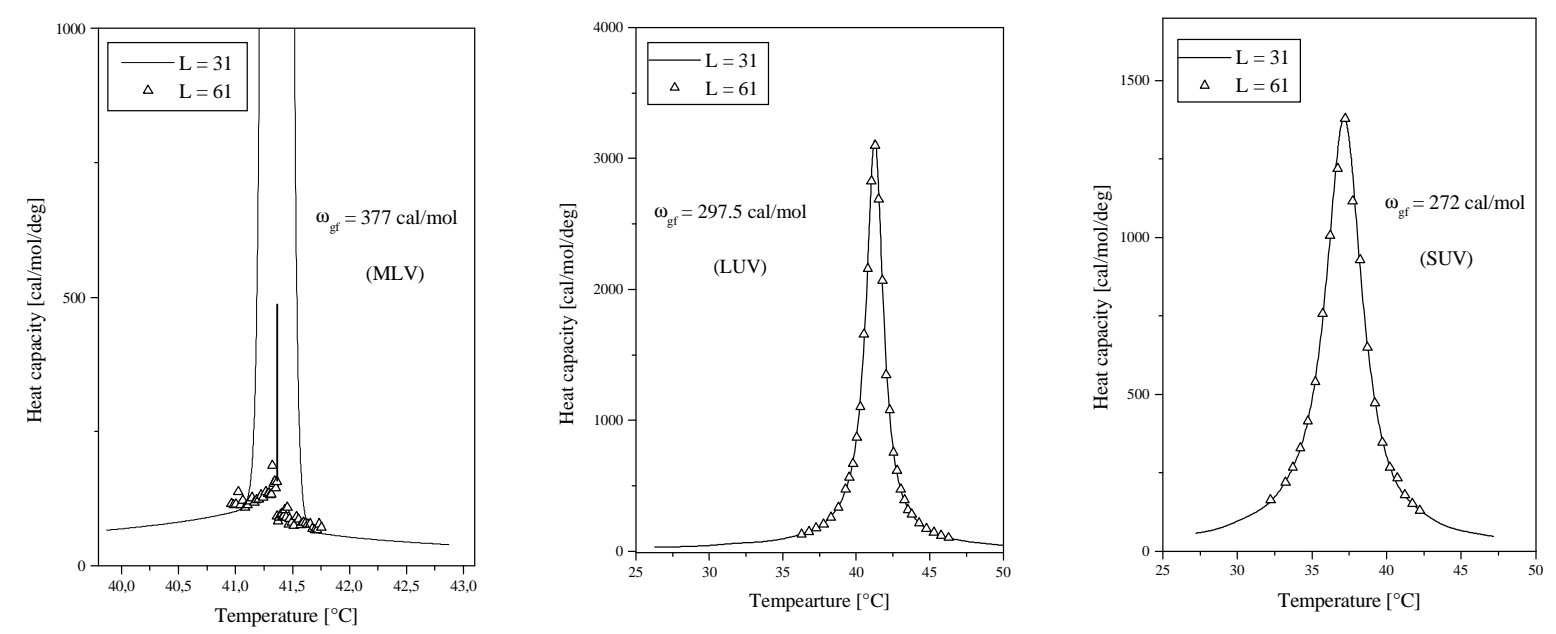

Figure 2.8. Heat capacity profiles calculated on an $\mathrm{L} \times \mathrm{L}$ hexagonal lattice. Lines: $\mathrm{L}=31$ (histogram results); Open triangles: $\mathrm{L}=61$ (results obtained by running a MC simulation for each temperature). The calculations were performed with the same parameters used to fit DPPC vesicle dispersions. Left: MLV; Center: LUV; Right: SUV. 
It is evident that for the fit parameters in the case of SUV (Figure 2.8 - right hand side) and LUV (Figure 2.8 - center), the 31.31 lattice represents the thermodynamic limit. However, in the case of MLV the exact number of $\omega_{g f}$ is obviously finite-size dependent. Larger matrices would yield a more accurate value for this case [39].

\subsection{Discussion}

Let us concentrate for a while on the importance of cluster formation in the lipid system. Figure 2.9 shows representative lipid configurations obtained from Monte Carlo simulations for temperatures below, at, and above the temperature corresponding to the mid-point of the chain-melting transition.

As shown, the thermal fluctuations give rise to the appearance of clusters of various sizes which are composed essentially of lipids in a single state (either gel or fluid). As the temperature changes towards the mid-point of the transition, the clusters composed of lipids in the minority state grow in size at the expense of the majority state, achieving their maximum size at the transition mid-point. The tendency to domain ${ }^{\#}$ formation results from the unfavorable interfacial energy between the gel and the fluid lipid states and becomes increasingly more pronounced as the interfacial energy increases and the transition becomes more competitive. On the other hand, the domain surface is complex, because the increased entropy of a more extended interface is energetically favorable. Thus, the unfavorable interfacial free energy (assumed here to be totally enthalpic, i.e. temperature independent) and the favorable entropy of an extended interface balance each other in a temperaturedependent manner. However, the higher the value of the enthalpic parameter $\omega_{g f}$, the larger the average domain size and the shorter the overall domain interface become.

\footnotetext{
\# Should not be confounded with the coexistence of two phases at a first-order phase transition. The heterogeneities we describe, result from the thermal fluctuations at equilibrium.
} 


$$
\mathrm{T}=\mathrm{T}_{\mathrm{m}}-2
$$

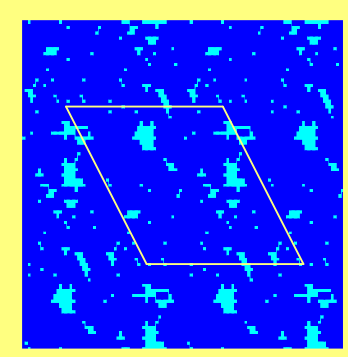

$$
\mathrm{T}=\mathrm{T}_{\mathrm{m}}
$$

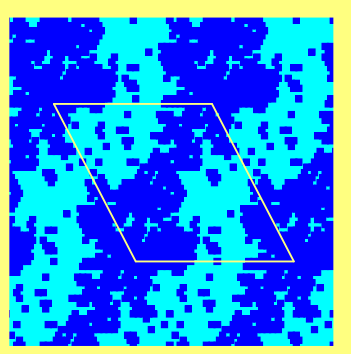

$$
\mathrm{T}=\mathrm{T}_{\mathrm{m}}+2
$$

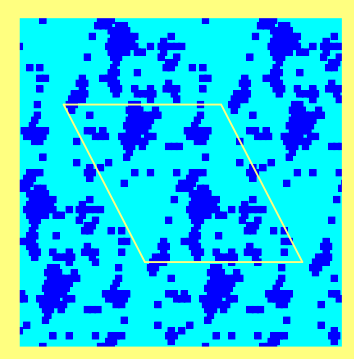

Figure 2.9. Monte Carlo snapshots at different temperatures for $\omega_{g f}=315 \mathrm{cal} / \mathrm{mol}$ (left: below $T_{m}$, center: at $T_{m}$ and right: above $T_{m}$ ). Monte Carlo simulations on a 31.31 matrix. The unit cell of the simulation is indicated by the rhombic box in the center of each picture.

A question arises: is it possible to detect membrane heterogeneity on a macro-scale? Indeed strong fluctuations develop on every scale, from molecules to the entire system, close to the critical point. Figure 2.10 shows domain formation in DMPC and DPPC monolayers close to their critical point [34].
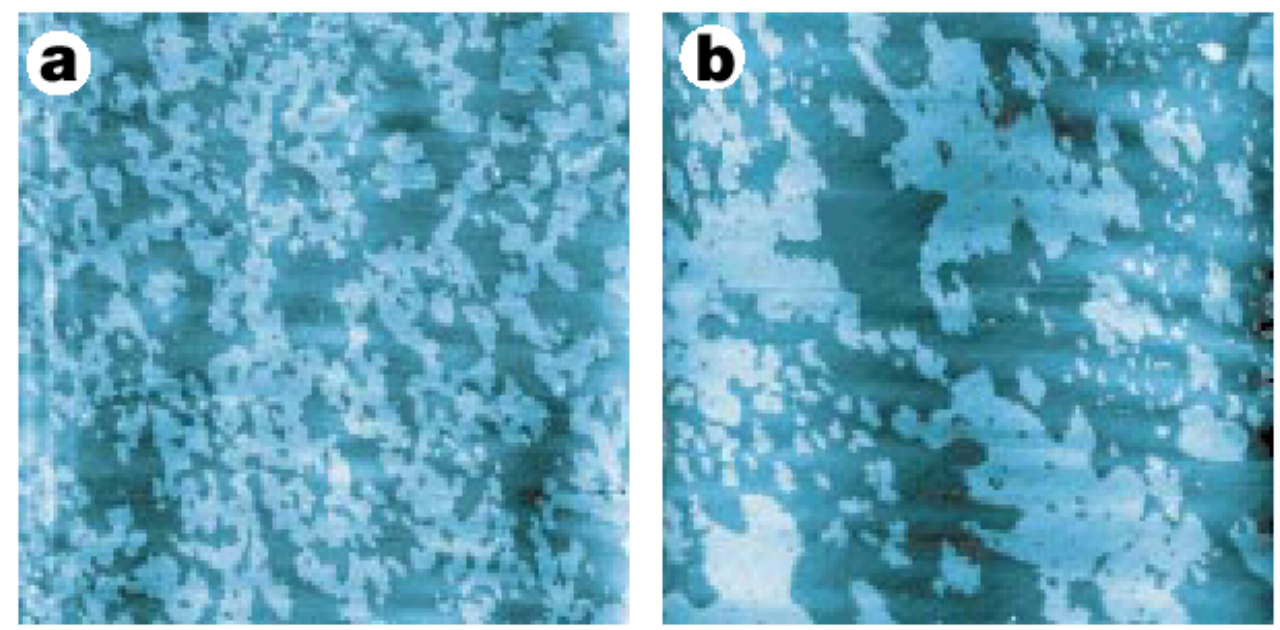

Figure 2.10. Images of (a) DMPC $\left(25 \times 25 \mu \mathrm{m}^{2}\right)$ and (b) DPPC $\left(20 \times 20 \mu \mathrm{m}^{2}\right)$ monolayers at their respective critical points. The monolayers have been transferred from an air-water interface to solid mica support and imaged by atomic force microscopy as a height difference. The height difference between the light and dark areas is about $5 \AA$ (from [34]). 
In single lipid monolayers domain coexistence has also been shown by fluorescence microscopy $[13,14]$. In the case of two-component lipid mixtures the direct visualization of the existence of coexisting lipid phases was detected by confocal fluorescence microscopy on giant unilamellar vesicles (GUV) [27] (Figure 2.11).

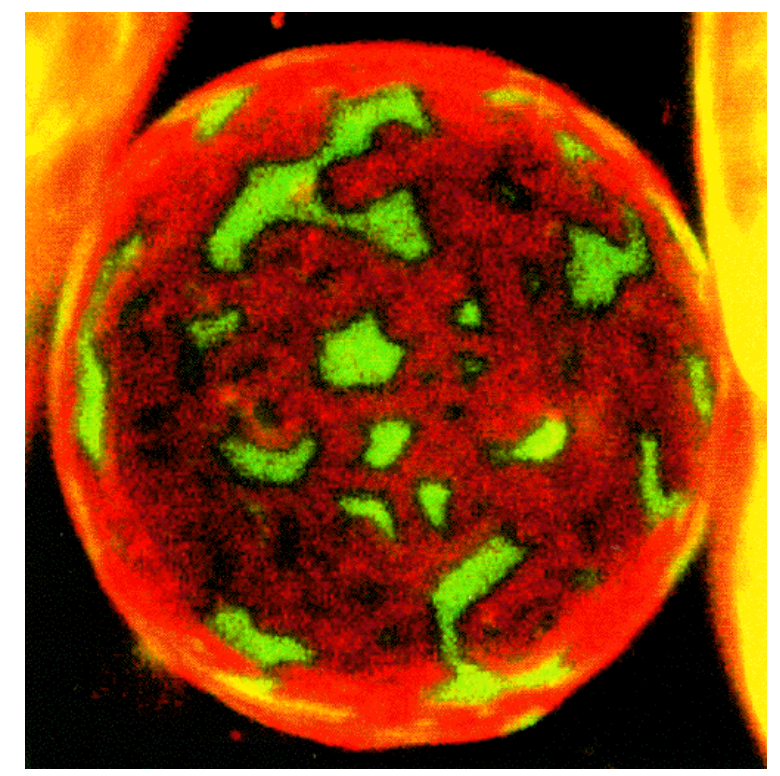

Figure 2.11. Visualization of phase separation in the binary lipid mixture of dilauroyl and dipalmitoyl phosphatidylcholine, DLPC/DPPC $=0.2 / 0.8$. The dark gel domains (DiI- $\mathrm{C}_{20}$ fluorescent probe) form a network on the vesicle surface, enclosing small islands of light fluid phase (Bodipy-PC fluorescent probe) (from [27]).

The strong thermal fluctuations in lipid membranes near their main phase transition may be linked to a variety of membrane functions such as passive permeability of small ions [2], enzyme and protein activity and protein binding [23]. Biological membranes contain many different lipid species that are probably organized heterogeneously in the form of domains or ,rafts“ [37] in the membrane plane.

Additionally, it should be noted that mechanical properties of the lipid membrane, like lateral compressibility and bending modulus, can also be obtained from the MC simulations for the two-state model. Using the experimentally established proportionality between the enthalpy and volume fluctuations close to the chain melting transition, one can derive simple relations between volume and area compressibilities and the heat capacity. The compressibility and elasticity display pronounced maxima at the phase transition, which 
can also be related to pronounced minima of the sound velocity in the lipid transition range $[19,22]$.

As demonstrated above (Figure 2.2), the cooperativity parameter influences the heat capacity profile significantly. This, together with the fact that the MC simulations are basically time-consuming, makes a real quantitative comparison between the $\mathrm{MC}$ simulation results and experimental heat capacities feasible only by applying the histogram method. However, in [38] the unknown cooperativity parameter is determined without using the histogram technique, but simply by calculating the average values over the time. Nevertheless, it is worth noting that such a procedure is very time consuming.

The histogram method of analyzing simulation data has a long history [see e.g. Ref.18 in 28]. This technique saves much CPU time, thus allowing quasi-analytical calculations. However, these calculations are correct only when the two sets of parameters of both measured and calculated histograms do not differ too much. For the determination of observables for a general set of parameters one requires a broad histogram, which covers most of the available phase space. This can be done by several methods: One can obtain the basic histogram at a critical point where fluctuations are large [22]. One can also combine many histograms obtained for different sets of parameters into one broad histogram by using reweighting and matching techniques $[40,3,9]$. An alternative approach leading to a broad histogram using a MC procedure different from the Metropolis algorithm was described by [6,41]. The multiple histogram method proposed by [15] uses the dynamical ensemble [11]. In order to construct our broad histogram, we used a histogram technique as described in [8] and matched the histograms using a method similar to that introduced by $[3,40]$.

Ising-like models have also been used previously to analyze lipid melting behavior $[38,39,19-22]$. In this chapter, we successfully combined the simplicity of the model with the histogram method and generated fits of the experimental heat capacity profiles of various vesicular preparations of DPPC. This was done by varying the interfacial cooperativity parameter $\omega_{g f}$ with constant values for the melting enthalpies for a monolayer system which is identical to assuming a bilayer with two uncoupled monolayers. In experimental systems, however, vesicles are usually not freely fluctuating membrane sheets but are rather located in vesicular systems of predefined geometry. Therefore, the 
fluctuations in the two monolayers are usually not uncoupled [20]. Lipids change their area by about $25 \%$ upon melting. An asymmetry of the areas of the two monolayers automatically induces a bilayer curvature. If the curvature is fixed, the area fluctuations on both monolayers are confined such that on average the area difference on both monolayers is constant. This implicitly results in a coupling of the fluctuations in both monolayers. A simple approach to account the curvature effects and the coupling of the monolayers was developed in [25].

\subsection{Conclusions}

The two-state model adopted for the lipid phase transition is very simple and transparent. It has only one parameter, which is not directly measured in an experiment. This effective interfacial energy parameter is obtained indirectly from the half-width of the heat capacity peak. For the description of the energy of a micro-state only two variables are required - the number of fluid lipids and the number of unlike near neighbor contacts. The two-sate model thus allows construction of a broad histogram of states, which is used for quasi-analytical calculation of heat capacity profiles. The information sampled in the broad monolayer histogram was first used to generate melting profiles of a bilayer system consisting of two uncoupled monolayers and to fit experimentally obtained DSC traces of different vesicle preparations.

\section{References}

[1] Bloom, M., Evans, E., and Mouritsen, O. G. (1991). Quarterly Reviews of Biophysics, Vol. 24, 293-397

[2] Boheim, G., Hanke, W., and Eibl, H. (1980). Proc. Natl. Acad. Sci. USA, Vol. 77, 3403-3407

[3] Bowen, P. et al. (1989). Physical Review B, Vol. 40, 7439

[4] Corvera, E., Laradji, M., and Zuckermann, M. L. (1993). Physical Review E, Vol. 47, 696 
[5] Cruizero-Hansson, L., Ipsen, J. H., and Mouritsen, O. G. (1990). Biochimica et Biophysica Acta, Vol. 979, 166

[6] de Oliveira, P. M. C., Penna, T. J. P., and Hermann, H. J. (1998). European Physical Journal B, Vol. 1, 205

[7] Doniach, S. (1978). Journal of Chemical Physics, Vol. 68, 4912

[8] Ferrenberg, A. M. and Swendsen, R. H. (1988). Physical Review Letters, Vol. 61, 2635

[9] Ferrenberg, A. M. and Swendsen, R. H. (1989). Physical Review Letters, Vol. 63, 1195

[10] Fisher, M. E. (1967). The theory of equilibrium critical phenomena, in Reports on Progress in Physics, Vol. 30, Part 2, see p. 671 and note that $J=\omega_{g f} / 2$

[11] Gerling, R. W. and Hüller, A. (1993). Zeitschrift für Physik, Vol. 90, 207

[12] Glauber, R. J. (1963). Journal of Mathematical Physics, Vol. 4, 294-307

[13] Grainger, D. W., Reichert, A., Ringsdorf, H., and Salasse, C. (1989). FEBS Letters, Vol. 252, 73-82

[14] Grainger, D. W., Reichert, A., Ringsdorf, H., and Salasse, C. (1990). Biochimica et Biophysica Acta, Vol. 1023, 365-379

[15] Hammrich, O. (1993). Zeitschrift für Physik B, Vol. 92, 501

[16] Hansen, J. P., Levesque, D., and Weis, J. J. (1979). Physical Review Letters, Vol. 43, 979

[17] Hansen, P. L., Miao, L., and Ipsen, J. H. (1998). Physical Review E, Vol. 58, 2311

[18] Heermann, D. W. (1990). Computer Simulation Methods in Theoretical Physics, $2^{\text {nd }}$ Edition, Springer, Berlin

[19] Heimburg, T. (1998). Biochimica et Biophysica Acta, Vol. 1415, 147

[20] Heimburg, T. (2000). Biophysical Journal, Vol. 78, 1154

[21] Heimburg, T. and Biltonen, R. L. (1996). Biophysical Journal, Vol. 70, 84 
[22] Heimburg, T. and Marsh, D. (1996). in Biological Membranes: A molecular perspective from computation and experiment, edited by K. M. Merz and B. Roux (Birkhäuser, Boston), pp. 405-462

[23] Heimburg, T., Angerstein, B., and Marsh, D. (1999). Biophysical Journal, Vol. 76, $2575-2586$

[24] Hill, T. L. (1960). An Introduction to Statistical Thermodynamics, Dover Publications, New York

[25] Ivanova, V. P. and Heimburg, T., submitted

[26] Janiak, M. J., Small, D. M., and Shipley, G. G. (1976). Biochemistry, Vol. 15, 45754580

[27] Korlach, J., Schwille, P., Webb, W. W. and Feigenson, G. W. (1999). Proc. Natl. Acad. Sci. USA, Vol. 96, 8461-8466

[28] Lee, J. and Kosterlitz, J. M. (1991). Physical Review B, Vol. 43, 3265-3277

[29] Marcelja, S. (1974). Journal of Chemical Physics, Vol. 60, 3599

[30] Mouritsen, O. G. (1990). in Molecular description of biological membrane components by computer aided conformational analysis, edited by R. Brasseur (CRC Press, Boca Raton), pp. 3-83

[31] Mouritsen, O. G. and Biltonen, R. L. (1992). In Protein-Lipid Interactions, A. Watts, editor, Elsevier, New York, 1-35

[32] Mouritsen, O. G. and Jorgensen, K. (1995). Molecular Membrane Biology, Vol. 12, 15

[33] Mouritsen, O. G. and Sperotto, M. M. (1992). In Thermodynamics of cell surface receptors, M. Jackson, editor, CRC Press, Boca Raton, FL. 127-181

[34] Nielsen, L. K., Bjornholm, T., and Mouritsen, O. G. (2000). Nature, Vol. 404, 352

[35] Pink, D. A. and Chapman, D. (1979). Proc. Natl. Acad. Sci. USA, Vol. 76, 1542

[36] Risbo, J., Sperotto, M. M., and Mouritsen, O. G. (1995). Journal of Chemical Physics, Vol. 103, 3643

[37] Simons, K. and Ikonen, E. (1997). Nature, Vol. 387, 569-572 
[38] Sugar, I. P., Biltonen, R. L., and Mitchard, N. (1994). in Methods in Enzymology, edited by L. Brand and M. L. Johnson, Academic Press, New York, Vol. 240, 569593

[39] Sugar, I. P., Thompson, T. E., and Biltonen, R. L. (1999). Biophysical Journal, Vol. 76, 2099

[40] Valleau, J. P. and Card, D. N. (1972). Journal of Chemical Physics, Vol. 57, 5457

[41] Wang, J.-S. (1999). European Physical Journal B, Vol. 8, 287

[42] Zhang, Z., Sperotto, M. M., Zuckermann, M. J., and Mouritsen, O. G. (1993). Biochimica et Biophysica Acta, Vol. 1147, 154 


\section{Monte Carlo simulations on mixed lipid/peptide systems}

\subsection{Introduction}

Insertion of transmembrane amphiphilic proteins or polypeptides into lipid membranes is known to have a considerable influence on the phase equilibria of the mixed system [28]. Usually this results in a shift in the heat capacity maximum and a broadening of the melting profile. Examples are the interaction of the integral band 3 protein of erythrocytes [21], the transferin receptor [19], and cytochrome $b_{5}$ with neutral lipids [7]. In particular the lipidprotein interactions often induce dramatic phase-separation phenomena. Moreover, the aggregation state of the proteins within the different lipid phases is strongly influenced by the interactions with the lipid bilayer. Band 3 protein aggregation within lipid domains in erythrocyte membranes has been reported [32]. The peptides gramicidin A and alamethicin form channels or pores that consist of dimers or aggregates [9]. Because of its effects on protein association or clustering the lipid state thus can be considered as a possible basis for the general control of protein function in biological membranes.

One of the theoretical guidelines proposed to relate protein-induced lipid-bilayer phase equilibria to basic physical properties of the lipid/protein interfacial contact is the concept of hydrophobic matching $[25,26,4]$ between the lipid-bilayer and protein hydrophobic thicknesses. This concept had some success in predicting phase diagrams for lipid bilayers reconstituted with proteins like bacteriorhodopsin, photosynthetic reaction center proteins, as well as band 3 protein [28]. In a related study, Zhang et al. [40] calculated the phase diagram and the heat capacity function for a pure lipid system undergoing a first-order phase transition at various compositions of an integral polypeptide that was assumed to occupy a site of the size of one lipid chain. The calculations predicted a closed phasecoexistence loop and a heat capacity function that develops a broad shoulder on the low temperature side, and broadens and shifts to lower temperatures with increasing peptide concentration.

In this chapter we use the two-state Ising model to describe the heat capacity profiles and the lateral distribution of lipids in the presence of small integral proteins. As in Chapter 2, a broad histogram of the distribution of states is constructed and used for quasi-analytical 
calculation of heat capacity profiles in order to compare with DSC traces measured on some model systems. As a consequence, the peptide aggregation properties in gel and fluid lipid phase are predicted. These predictions were tested in atomic force microscopy measurements. Additionally, the simulations demonstrate how the different mixing of the peptide with both lipid phases can induce fluctuations in the membrane plane.

\subsection{Energy of the system and distribution of states}

Our system is a lipid monolayer containing a small peptide similar in size to a lipid, e.g. an integral $\alpha$-helical peptide. Again, as described in Section 2.3, the heat capacity can be determined from the fluctuations of the enthalpy using the fluctuations-dissipation theorem (Equation 2.6). To generate such fluctuations Monte Carlo simulations employing two components, lipids and peptides, have to be performed. As in Equation (2.4), the Gibbs free energy of a given configuration is

$$
\Delta G\left(n_{f}, n_{g f}, n_{g p}, n_{f p}\right)=n_{f} \cdot(\Delta H-T \cdot \Delta S)+n_{g f} \cdot \omega_{g f}+n_{g p} \cdot \omega_{g p}+n_{f p} \cdot \omega_{f p}
$$

where $\omega_{g p}$ and $\omega_{f p}$ are effective interaction energies between gel and peptide, and fluid and peptide, respectively.

$$
\begin{aligned}
& \omega_{g p}=\varepsilon_{g p}-\left(\varepsilon_{g g}+\varepsilon_{p p}\right) / 2 \\
& \omega_{f p}=\varepsilon_{f p}-\left(\varepsilon_{f f}+\varepsilon_{p p}\right) / 2
\end{aligned}
$$

The lipids and the peptides form a hexagonal lattice. We consider peptide molecules which are small enough to occupy only a single lattice site. The interactions and the arrangement of the molecules is schematically shown in the cartoon below.
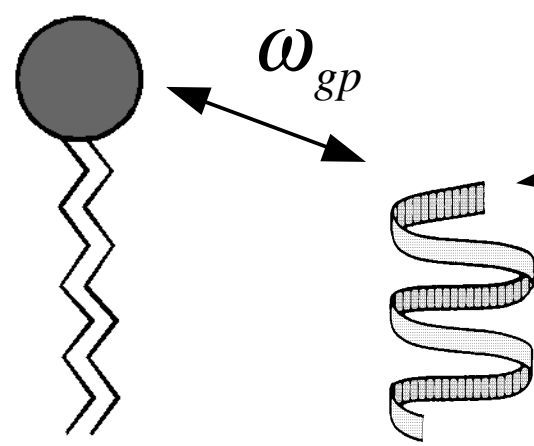

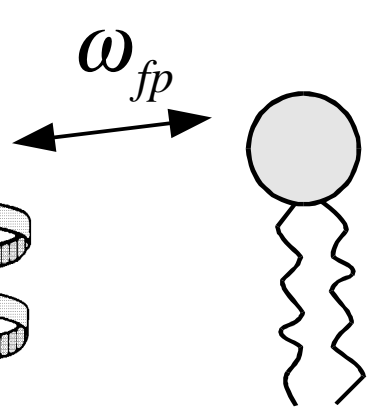

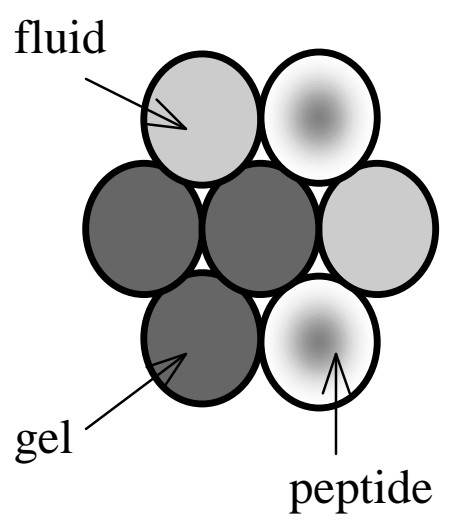


Unlike in Chapter 2, in this system we have two further interaction energies, $\omega_{g p}$ and $\omega_{f p}$. The Monte Carlo algorithm we use can be described briefly as follows:

- Pick a lattice point at random.

- If it is a lipid, change its state (and proceed as described in Section 2.3).

- If it is a peptide:

$\Rightarrow$ Pick a lipid at random.

$\Rightarrow$ Exchange it with the peptide.

$\Rightarrow$ Calculate the Gibbs energy difference between the new and the old matrix configuration

$$
\delta G=\delta G^{l i p i d}+\delta G^{\text {peptide }}
$$

where $\delta G^{l i p i d}=\left(\Delta n_{g f}^{l i p i d} \cdot \omega_{g f}+\Delta n_{g p}^{l i p i d} \cdot \omega_{g p}+\Delta n_{f p}^{l i p i d} \cdot \omega_{f p}\right)$ and $\Delta n_{g f, g p, f p}^{l i p i d}$ denote the increase of the respective unlike nearest neighbor contacts of the lipid; $\delta G^{\text {peptide }}$ is given by a similar expression, accounting for the change of the unlike nearest neighbor contacts of the peptide.

$\Rightarrow$ Calculate the probability for the change

$$
P=\frac{K(T)}{1+K(T)}, \quad \text { with statistical weights } \quad K(T)=\exp \left(-\frac{\delta G}{R T}\right)
$$

$\Rightarrow$ Generate a random number RAN and compare it with the calculated probability.

$\Rightarrow$ Make a decision (YES or NO) for the exchange of the lipid and peptide.

$$
\begin{array}{lll}
R A N \leq P & \mapsto & Y E S \\
R A N>P & \mapsto & N O
\end{array}
$$

Additionally, the two-component character of the system implies a diffusion of one component into the other. However, our simulations are intended to be equilibrium calculations and thus we will not account for the peptide diffusion*. The unknown interaction energies have to be determined by comparison with experimental heat capacity profiles. For this comparison again the histogram method for Monte Carlo data analysis [6]

\footnotetext{
* This can be done by exchanging only nearest neighbors [17].
} 
will be applied. Thus, one needs to construct a broad histogram of the distribution of states of the system described. As described in Section 2.5, each MC simulation produces a probability distribution of states

$$
P\left(n_{p}, n_{f}, n_{g f}, n_{f p}, n_{g p}, \zeta\right)=\frac{\Omega\left(n_{p}, n_{f}, n_{g f}, n_{f p}, n_{g p}\right) \cdot \exp \left(-\Delta G\left(n_{f}, n_{g f}, n_{f p}, n_{g p}, \zeta\right) / R \cdot T\right)}{\sum_{n_{f}, n_{g f}} \Omega\left(n_{p}, n_{f}, n_{g f}, n_{f p}, n_{g p}\right) \cdot \exp \left(-\Delta G\left(n_{f}, n_{g f}, n_{f p}, n_{g p}, \zeta\right) / R \cdot T\right)}
$$

where $\zeta$ is the set of simulation parameters $\left(n_{p}, T, \Delta H, T_{m}, \omega_{g f}, \omega_{f p}, \omega_{g p}\right), n_{f}$ is the number of fluid lipids; $n_{g f}, n_{f p}$ and $n_{g p}$ are the respective numbers of gel-fluid, fluid-peptide and gel-peptide contacts. Here $n_{p}$ indicates the number of the peptides on the lattice and hence $0 \leq n_{p} \leq n$, where $n$ is the total number of lattice sites.

The degeneracy of the states $\Omega\left(n_{p}, n_{f}, n_{g f}, n_{f p}, n_{g p}\right)$ does not depend on the parameters of the MC simulation. It depends, however, on $n_{p}$ which determines the fractions of the two components - lipids and peptides. As will be demonstrated below, the construction of the broad histogram for the mixed lipid-peptide system is much more time consuming than the construction of the two-dimensional histogram (Chapter 2). Therefore, we performed MC simulations at a fixed peptide fraction $f_{p}=n_{p} / n=0.1$, to slightly reduce the complexity of the problem. As we will show later (Section 3.6), this value is justified by the fact that in the experiment the peptide cross-section is somewhat larger than that of a single lipid.

The sampled four-dimensional probability distribution of states of a mixed system with peptide fraction $f_{p}=0.1$ was captured into a histogram with $N$ bin, i.e. the whole phase space was divided into small volumes of a size $\Delta f \times \Delta \bar{n}_{g f} \times \Delta \bar{n}_{f p} \times \Delta \bar{n}_{g p}$ and the number of events in each bin was counted during the simulation. Here, $f$ denotes the fluid fraction defined as a fraction of the amount of lipids, i.e. $f=n_{f} /\left(n \cdot\left(1-f_{p}\right)\right) ; \bar{n}_{g f}, \bar{n}_{f p}$ and $\bar{n}_{g p}$ are the fractions of the mean number of unlike nearest neighbor interactions defined per lattice site. Since $n_{f p}+n_{g p}+2 \cdot n_{p p}=6 \cdot n_{p}$, where $n_{p p}$ is the number of peptide-peptide contacts, it turns out that the following inequality for the lipid-peptide contacts is valid:

$$
0 \leq n_{f p}+n_{g p} \leq 6 \cdot n_{p}
$$

This implies that at a peptide fraction $f_{p}=0.1$, the mean number of fluid-peptide and gelpeptide contacts per lattice site are limited in the interval $[0,0.6]$, i.e. $0 \leq \bar{n}_{f p} \leq 0.6$ and 
$0 \leq \bar{n}_{g p} \leq 0.6$. For the fluid fraction we have $0 \leq f \leq 1$ and, as it was demonstrated in Section 2.5, for the mean number of gel-fluid contacts per lattice site one has $0 \leq \bar{n}_{g f} \leq 2$.

We have chosen bins with the following dimensions: $\Delta f=\Delta \bar{n}_{f p}=\Delta \bar{n}_{g p}=0.01$ and $\Delta \bar{n}_{g f}=0.02$, as a compromise, because: On one hand, a histogram with smaller bins is more accurate and describes both the distribution of states and the phase transition, more precisely. On the other hand, the size of the bins is inversely proportional to the histogram size and hence to the CPU time needed to generate the histogram (more bins have to be visited during the random walk). In Chapter 2 we have shown, that the two-dimensional histogram with $\Delta f=\Delta \bar{n}_{g p}=0.01$ is sufficient to describe the melting transition of a singlecomponent lipid system. Here, we adopted a compromise and took $\Delta \bar{n}_{g f}=0.02$, in order to reduce the number of bins. The values $\Delta \bar{n}_{f p}=\Delta \bar{n}_{g p}=0.01$ correspond to $\Delta n_{f p}=\Delta n_{g p} \approx 9$ on $31 \cdot 31$ matrix. Thus, the whole phase space is divided in $N=100 \cdot 50 \cdot 60 \cdot 60=18 \cdot 10^{6}$ bins. The inequality (3.4) shows that the available phase space actually contains $9 \cdot 10^{6}$ bins. Finally, the question whether a certain bin size is precise enough or not, is answered by a comparison between calculated heat capacity profiles from the histogram and obtained directly by running MC simulations. Such examples will be given below.

A single measured histogram produced by one MC simulation is shown in Figure 3.1. The histogram is four-dimensional which is difficult to present on a simple picture. However, the kind of presentation given in Figure 3.1 gives an impression of the complexity of the problem. The left-hand side of Figure 3.1 shows a projection of the four-dimensional histogram in the two-dimensional plane $\left(f, \bar{n}_{g f}\right)$, i.e., shown are all events of generated matrix configuration with fluid fraction $f$ and mean number of gel-fluid contacts per matrix site $\bar{n}_{g f}$ independent on the numbers of fluid-peptide and gel-peptide contacts. Each point on this plot corresponds to a two-dimensional histogram in the plane $\left(\bar{n}_{f p}, \bar{n}_{g p}\right)$, as demonstrated in Figure 3.1 (right-hand side). The simulation was performed at a temperature corresponding approximately to the heat capacity maximum of this lipidpeptide mixture. In spite of the fact that the thermal fluctuations at this temperature are large, the phase space explored by the simulation is only about $0.27 \%$ of the whole phase space. For comparison, in the case of a single component lipid system a typical histogram sampled at $T_{m}$ explored about $10 \%$ of the phase space. Thus, in order to construct a 
practically useful broad four-dimensional histogram, much more distributions at different sets of parameters have to be sampled, than for the two-dimensional case.
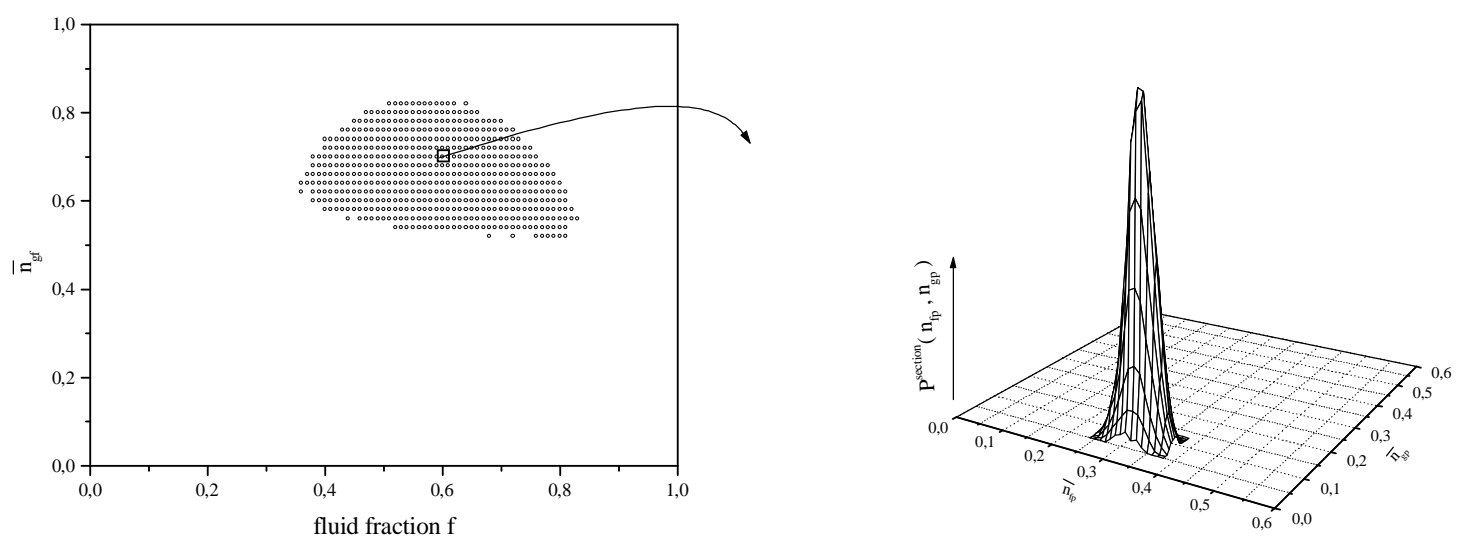

Figure 3.1. Four-dimensional probability distribution of states, produced by a single MC simulation on a mixed lipid-peptide monolayer, with the following set of parameters: $n_{p}=0.1, T=308.3 \mathrm{~K}, \omega_{g f}=\omega_{f p}=300 \mathrm{cal} / \mathrm{mol}$ and $\omega_{g p}=600 \mathrm{cal} / \mathrm{mol}$. Left: projection of the histogram in the $\left(f, \bar{n}_{g f}\right)$-plane. Right: section in the $\left(\bar{n}_{f p}, \bar{n}_{g p}\right)$-plane. The simulation contained $2 \cdot 10^{6} \mathrm{MC}$ cycles and was performed on a $31 \cdot 31$ matrix.

\subsection{Simple case of a gel-like peptide}

The broad two-dimensional histogram presented in Chapter 2 however, allows to calculate a special case of lipid-peptide system, where the peptide resembles either the gel or the fluid lipid state. In the following let us assume that the peptide has similar properties to those of a gel lipid. Such a case was considered in [12]. In fact, the peptides differ from the gel lipids only in that the peptides can not melt. This implies that the interfacial energy of a gel lipid with a peptide is similar to the contact between two gel lipids ( $\omega_{g p}=0$ - hence, the energy of the system does not depend on the gel-peptide contacts, $n_{g p}$ ), whereas a fluid lipid-peptide contact contributes to the overall Hamiltonian with $\omega_{f p}=\omega_{g f}$. This case is schematically shown in Figure 3.2. 


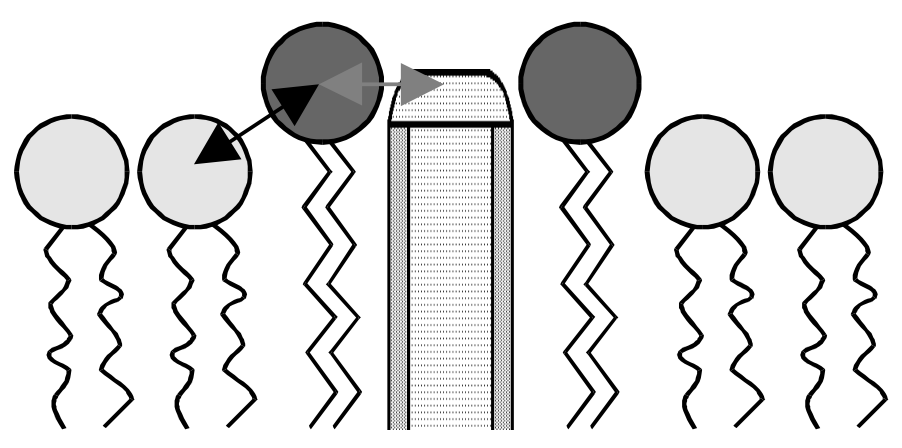

Figure 3.2. Scheme of the nearest neighbor interactions in a lipid monolayer containing gel lipids, fluid lipids and gel-like peptides. Shown are gel-fluid contacts and gel-peptide contacts.

The Gibbs free energy for a given configuration is then expressed in a simplified manner by:

$$
\Delta G\left(n_{f}, n_{g f}, n_{f p}\right)=n_{f} \cdot(\Delta H-T \cdot \Delta S)+n_{g f}^{f p} \cdot \omega_{g f}
$$

where $n_{g f}^{f p}=n_{g f}+n_{f p}$. With this expression the partition coefficients for this system can be derived from the lipid monolayer histogram.

$$
P\left(n_{f}, n_{g f}^{f p}, \zeta, n_{p}\right)=\frac{\Omega\left(n_{f}, n_{g f}^{f p}\right) \cdot \frac{\left(n_{g}+n_{p}\right) !}{n_{g} ! \cdot n_{p} !} \exp \left(-\frac{\Delta G\left(n_{f}, n_{g f}^{f p}, \zeta\right)}{R T}\right)}{\sum_{n_{f}} \sum_{n_{g f}^{t p}} \Omega\left(n_{f}, n_{g f}^{f p}\right) \cdot \frac{\left(n_{g}+n_{p}\right) !}{n_{g} ! \cdot n_{p} !} \exp \left(-\frac{\Delta G\left(n_{f}, n_{g f}^{f p}, \zeta\right)}{R T}\right)}
$$

where $n_{g f}^{f p}=n_{g f}+n_{f p}$ and $\zeta$ is the set of parameter $\left[T, \Delta H, T_{m}, \omega_{g f}\right]$. Equation (3.6) resembles Equation (2.8) except for an additional factorial term, $\left(n_{g}+n_{p}\right) ! / n_{g} ! \cdot n_{p}$ ! , which accounts for the number of ways to arrange $n_{p}$ gel-like peptides on the sites for gel and peptide in a given configuration. The heat capacities derived from this expression for different peptide concentrations, $n_{p}$, are given in Figure 3.3. When increasing the peptide concentration, the $C_{p}$-profiles are shifted to higher temperatures and are asymmetrically broadened, in agreement with previous results [12]. For comparison, the results from a two component Monte Carlo simulation with identical parameters based on Equation (2.6) are also given in Figure 3.3 (solid symbols). They are in exact agreement with the solid line obtained from the histogram (Equation (3.6)). Also shown in Figure 3.3 are some Monte 
Carlo snapshots obtained from simulations on a monolayer containing $10 \mathrm{~mol} \%$ gel-like peptides at various temperatures. It can be seen that the asymmetry of the heat capacity profiles corresponds to an aggregation of the gel-like peptides in the fluid lipid membrane due to unfavorable fluid-peptide contacts. In fact, at high temperatures the presence of gellike peptides causes „freezing“ of their lipid neighbors, since the gel-peptide contacts are energetically favorable. On the other hand, when the temperature increases, more and more lipids melt, since $T \cdot \Delta S$ is large and $(\Delta H-T \cdot \Delta S)<0$. The interplay between these two factors is the reason for large fluctuations at $T>T_{m}$, which causes the shoulder in the heat capacity profile (Equation 2.6).

The opposite case of fluid-like peptide also can be calculated from the two-dimensional histogram of a single lipid membrane in analogy to the just presented case. The partition coefficients are then given by

$$
P\left(n_{f}, n_{g f}^{g p}, \zeta, n_{p}\right)=\frac{\Omega\left(n_{f}, n_{g f}^{g p}\right) \cdot \frac{\left(n_{f}+n_{p}\right) !}{n_{f} ! \cdot n_{p} !} \exp \left(-\frac{\Delta G\left(n_{f}, n_{g f}^{g p}, \zeta\right)}{R T}\right)}{\sum_{n_{f}} \sum_{n_{g f}^{g p}} \Omega\left(n_{f}, n_{g f}^{g p}\right) \cdot \frac{\left(n_{f}+n_{p}\right) !}{n_{f} ! \cdot n_{p} !} \exp \left(-\frac{\Delta G\left(n_{f}, n_{g f}^{g p}, \zeta\right)}{R T}\right)}
$$

where $n_{g f}^{g p}=n_{g f}+n_{g p}$ and $\zeta$ is the set of parameter $\left[T, \Delta H, T_{m}, \omega_{g f}\right]$.

The heat capacity profiles are given in Figure 3.4. The $C_{p}$-traces of this mixture are shifted to lower temperatures and they develop a shoulder in the low temperature range. The simulation snapshots show that the peptides aggregate only in the lipid gel phase, but mix well with the fluid phase.

The two cases of gel- and fluid-like peptide, presented on Figure 3.3 and 3.4 can be easily distinguished. This allows to resolve qualitatively peptide cluster formation properties in both lipid phases. 


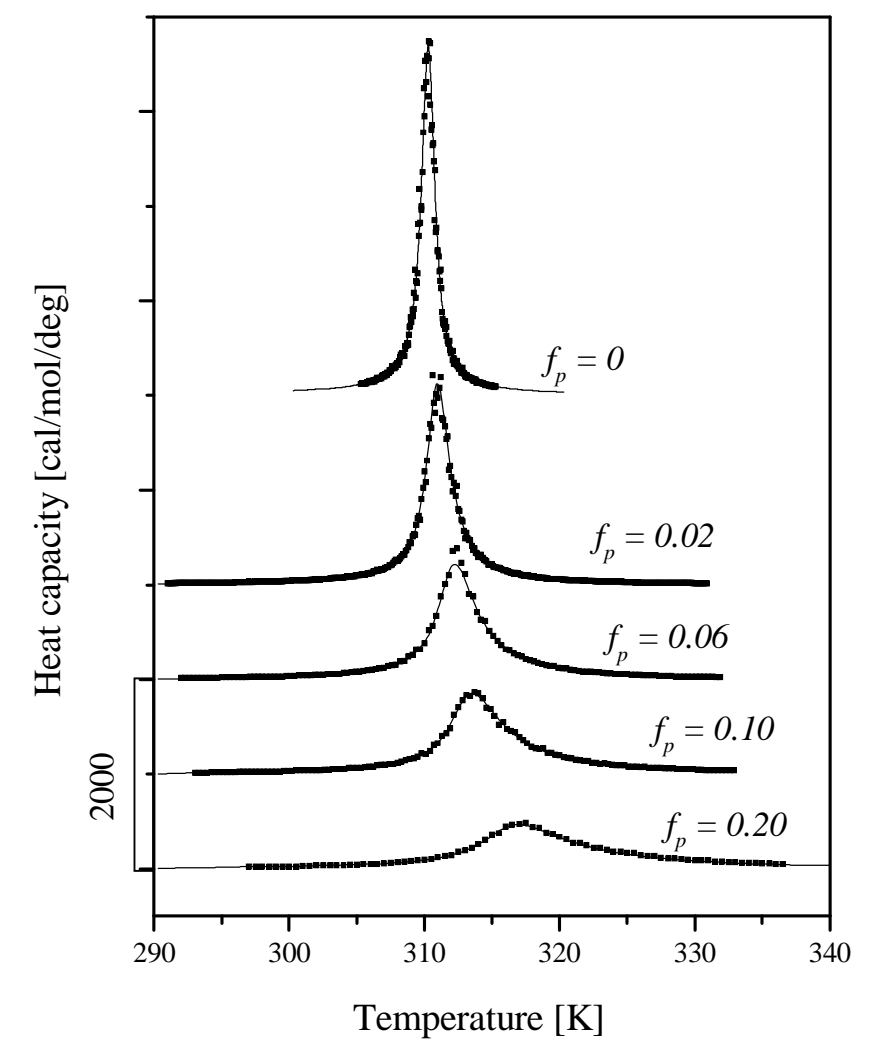

$$
\mathbf{T}=\mathbf{T}_{\mathbf{m}}
$$

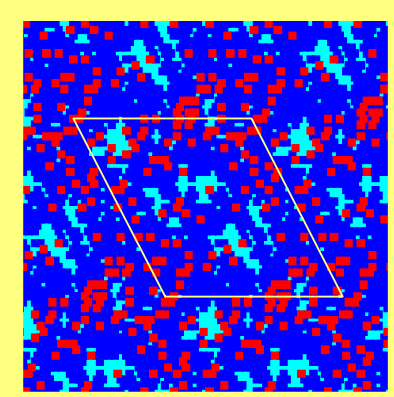

$\mathbf{T}=\mathbf{T}_{\mathrm{m}}+\mathbf{5}$

$\mathbf{T}=\mathbf{T}_{\mathrm{m}}+\mathbf{1 0}$

Figure 3.3. Top: Calculated heat capacity profile of a membrane containing various fractions of a gel-like peptide. Solid lines: calculated from (3.6). Small squares: calculated by running two component Monte Carlo simulations at each temperature. Bottom: Representative Monte Carlo snapshots of the lipid matrix containing 10mol\% gel-like peptides at several temperatures. Blue dots: gel lipids; cyan dots: fluid lipids; red dots: peptides. Simulations on a 31.31 matrix with periodic boundary conditions. Here and further, the unit cell of the simulation is indicated by the rhombic box in the center of the picture. 


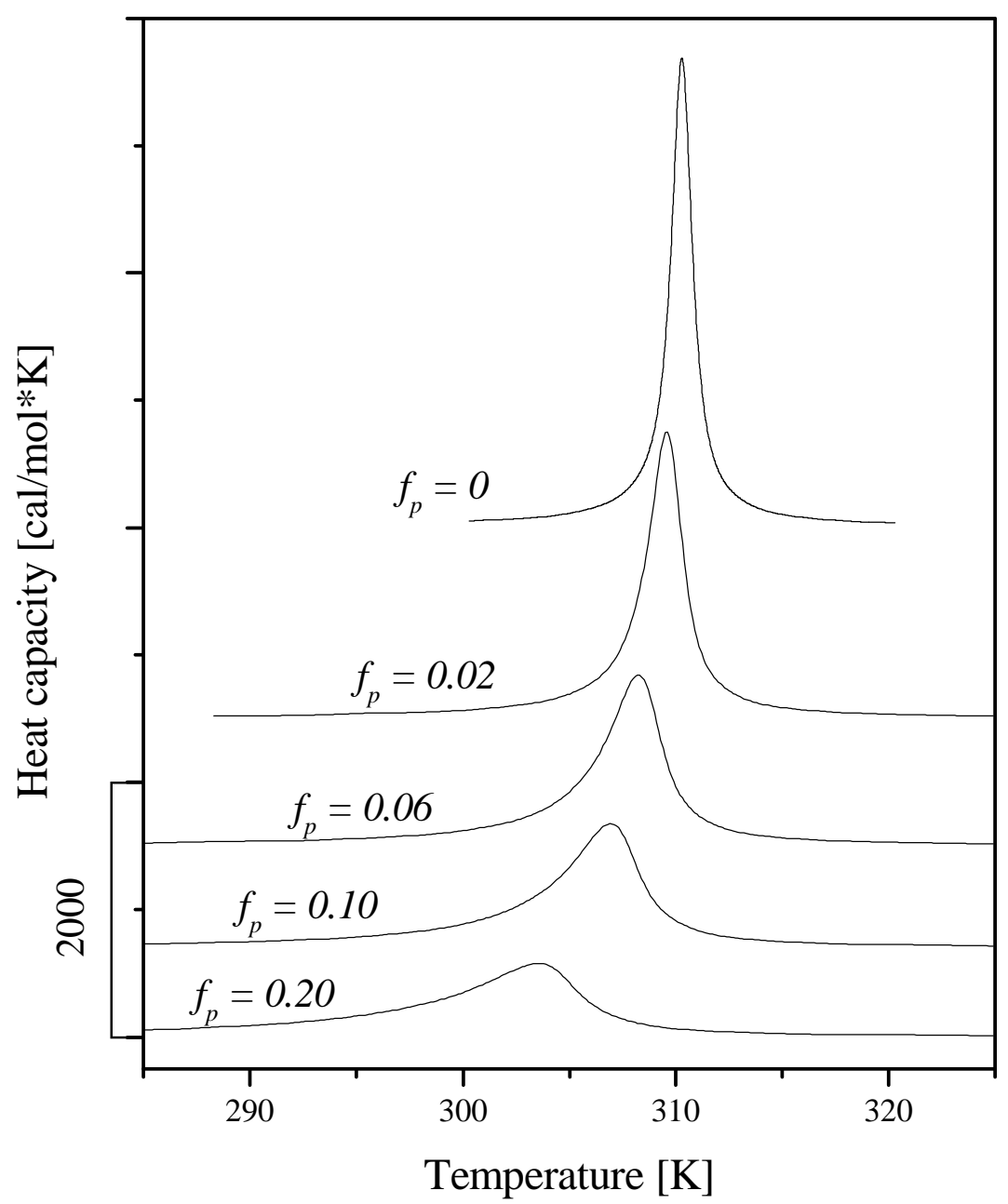

$\mathbf{T}=\mathbf{T}_{\mathrm{m}}-\mathbf{1 0}$

$\mathbf{T}=\mathbf{T}_{\mathbf{m}}-\mathbf{5}$

$\mathbf{T}=\mathbf{T}_{\mathbf{m}}$
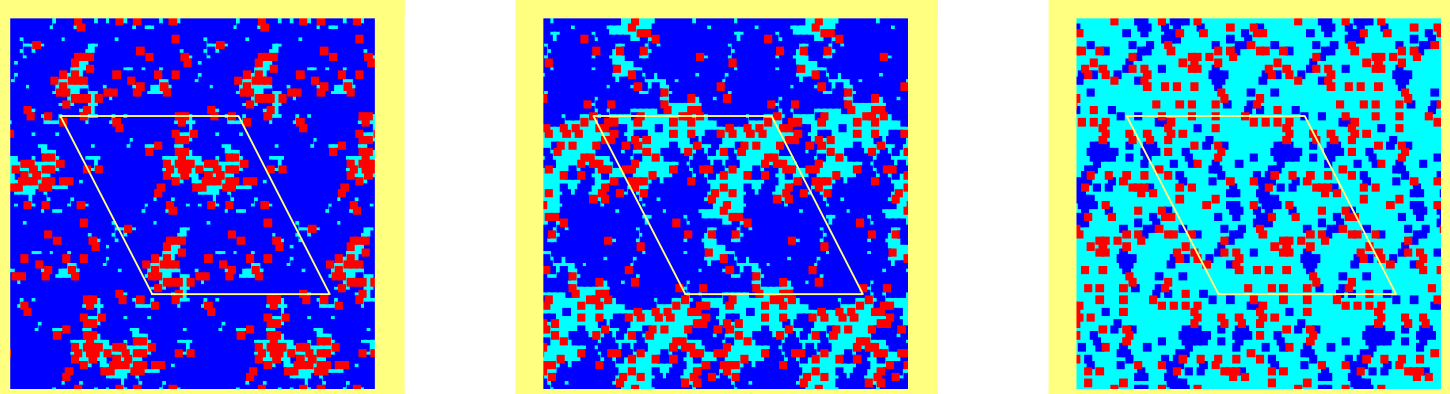

Figure 3.4. Top: Calculated heat capacity profile of a membrane containing various fractions of a fluid-like peptide (from Equation (3.7)). Bottom: Representative Monte Carlo snapshots of the lipid matrix containing 10mol\% fluid-like peptides at several temperatures. 


\subsection{Construction of a broad four-dimensional histogram}

The density of states $\Omega\left(n_{f}, n_{g f}, n_{f p}, n_{g p}\right)$ is a symmetrical function by the change $n_{f} \rightarrow n-n_{f}$, i.e.,

$$
\Omega\left(n_{f}, n_{g f}, n_{f p}, n_{g p}\right)=\Omega\left(n-n_{f}, n_{g f}, n_{g p}, n_{f p}\right)
$$

Because, the number of the gel-fluid contacts remains unchanged, but the fluid-peptide contacts become gel-peptide contacts and vice versa when we exchange each gel lipid to a fluid lipid and each fluid lipid to a gel lipid.

This symmetry was exploited to reduce the number of histograms required for constructing the broad histogram. We performed MC simulations at conditions $\omega_{f p} \leq \omega_{g p}$ only, i.e., since the gel-peptide contacts are unfavorable it is assumed that the peptide will mix predominantly with the fluid phase so that mostly configurations with large numbers of fluid-peptide contacts and less gel-peptide contacts are generated. Then, the remaining part of the (accessible) phase space can be explored using the symmetry of the distribution function.

To get an idea of reasonable values for the interaction energies, $\omega_{g p}$ and $\omega_{f p}$, we calculated some heat capacity profiles simply by running MC simulations at each desired temperature and compared them with measured DSC traces on mixed model systems. After that, we sampled histograms at different temperatures varying the interaction energies in the interval which was found suitable. The matching method used to combine the information of all measured histograms into one is the same as described in Section 2.5. The overlap was defined as the joint part of the phase explored by both neighboring histograms. The scaling ratio was then determined from the four-dimensional volumes in the overlapping region. The details about matching and parameters sets at which the histograms were sampled are listed in the Appendix. 


\subsection{Model simulation results}

\subsubsection{Limit cases}

Once sampled, the broad four-dimensional histogram can be used to calculate heat capacity profiles without running MC simulations for each set of parameters. Here we demonstrate some typical situations.

First, let's consider a case where $\omega_{f p}=\omega_{g p}=0$, i.e., the incorporated peptides mix ideally in both lipid phases. The heat capacity profile of such a system is given in Figure 3.5. The melting temperature of the mixed system is the same as of the pure lipid system. The heat capacity profile remains symmetric, but the transition cooperativity decreases. Additionally, some matrix snapshots are shown, below, at and above the transition temperature, $T_{m}$. In this case, the peptide is predominantly located at the interface between gel and fluid domains since this is energetically favorable. Thus, the presence of peptides decreases the cooperativity of the lipid transition - they prevent formation of large domains. This case is very similar to the case of cholesterol/lipid mixtures at low cholesterol content. The measured heat capacity traces of mixtures of DMPC with cholesterol at cholesterol fractions $x_{c}<0.1$ are simply broadened, but remain symmetric [10]. For the cholesterol molecule it is known that at such low concentrations no macroscopic phase separation between phases of different cholesterol content occurs. Rather, cholesterol is enriched in the interfaces of fluid and gel lipid domains, thus reducing the transition cooperativity $[3,27]$. The latter agrees well with our simulation results (see the MC snapshots in Figure 


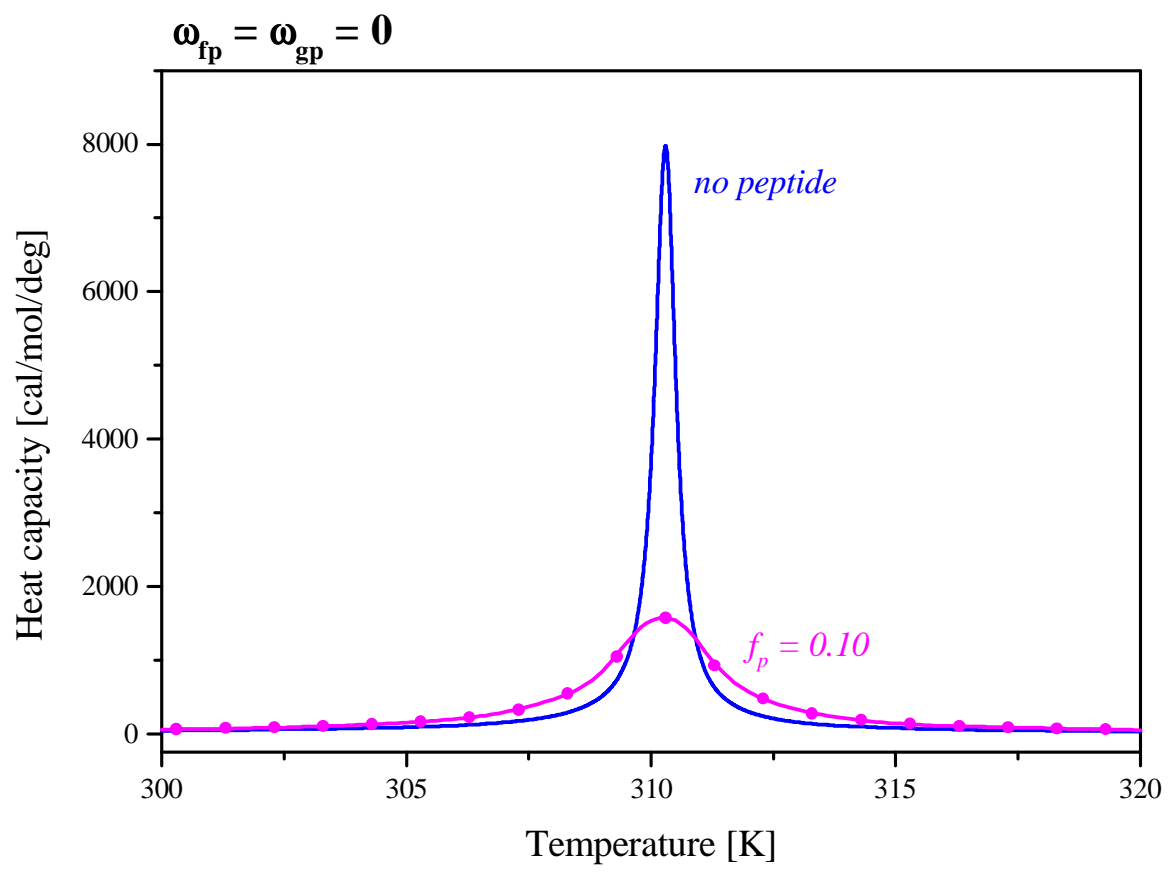

$\mathbf{T}=\mathbf{T}_{\mathbf{m}}-\mathbf{3}$

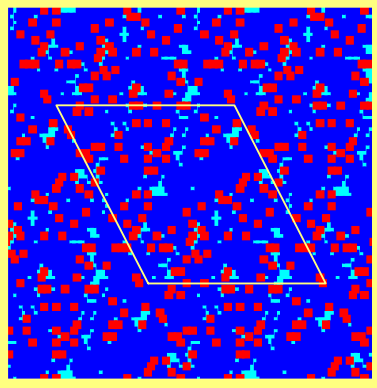

$\mathbf{T}=\mathbf{T}_{\mathbf{m}}$

$\mathbf{T}=\mathbf{T}_{\mathrm{m}}+\mathbf{3}$

Figure 3.5. Top: Calculated heat capacity profile of a membrane containing $10 \mathrm{~mol} \%$ peptide which mixes well with both lipid phases, $\omega_{f p}=\omega_{g p}=0$, $\omega_{g f}=315 \mathrm{cal} / \mathrm{mol}$. Lines: histogram results; blue: pure lipid system; pink: mixture. Solid circles: calculation from a two-component MC simulation. Bottom: Representative Monte Carlo snapshots of the lipid-peptide matrix at various temperatures. 
Another important limit case we would like to consider is a peptide which is very well soluble in one lipid phase but interacts extremely unfavorably with the other. This case is an extension of the example of gel- or fluid-like peptide (Section 3.3). The heat capacity profile of a system, in which the peptide interacts extremely well with the fluid lipid phase but demixes strongly from the gel phase is shown in Figure 3.6.
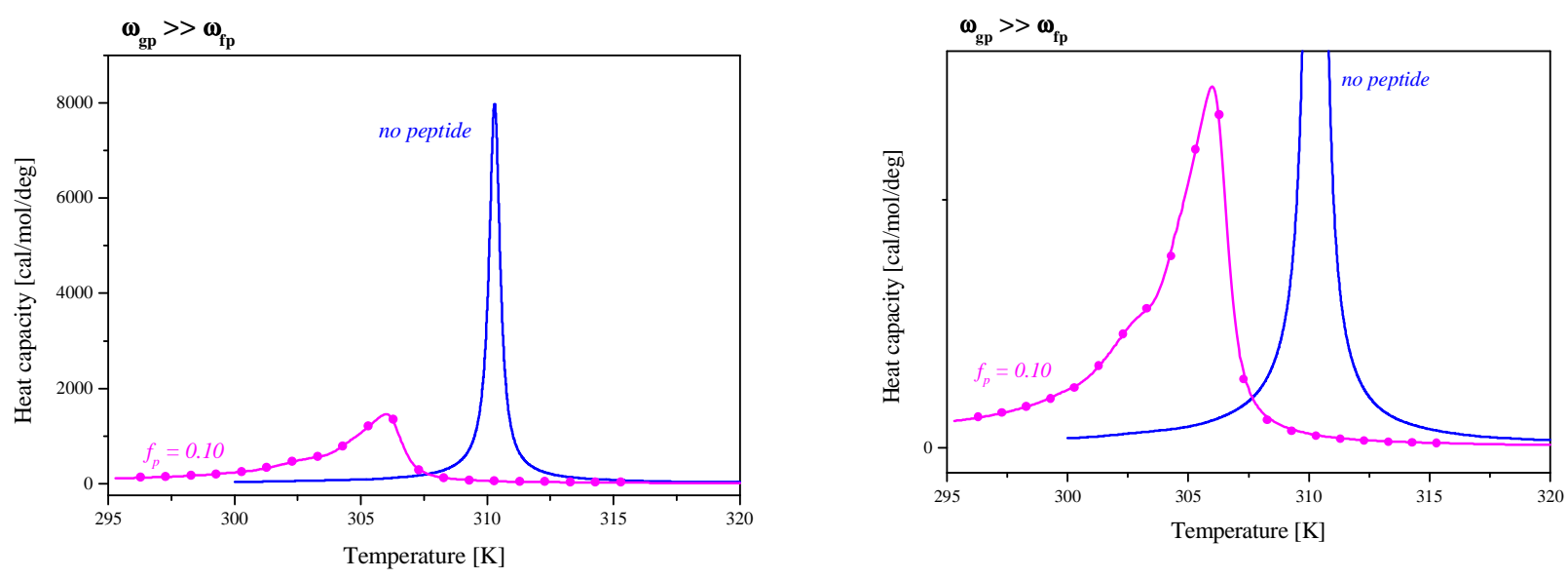

$$
\mathbf{T}=\mathbf{T}_{\mathbf{m}}-\mathbf{1 5}
$$

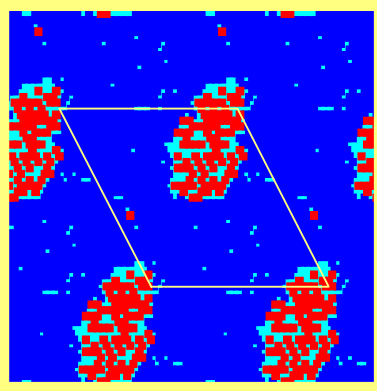

$\mathbf{T}=\mathbf{T}_{\mathbf{m}}-\mathbf{5}$

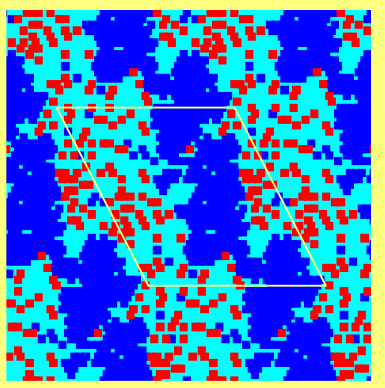

$\mathbf{T}=\mathbf{T}_{\mathbf{m}}$

Figure 3.6. Top left: Calculated heat capacity profile of a membrane containing 10 mol\% peptide which mixes well with the fluid phase, but demixes strongly from the gel lipid phase, $\omega_{f p}=0, \omega_{g p}=900 \mathrm{cal} / \mathrm{mol}, \omega_{g f}=315 \mathrm{cal} / \mathrm{mol}$. Top right: Enlarged heat capacity profile of the mixed system. Lines: histogram results; blue: pure lipid system; pink: mixture. Solid circles: calculation from a two-component MC simulation. Bottom: Representative Monte Carlo snapshots of the lipid-peptide matrix at various temperatures. 
It is evident that in comparison to the fluid-like peptide (Figure 3.4), there is much stronger peptide aggregation in the low-temperature gel phase. Accordingly, the suppression of the phase transition temperature is larger $\left(T_{m}^{\text {lipid }}-T_{m}^{\text {mixture }} \approx 5 K\right)$. The calculated heat capacity profile develops a very long shoulder with a certain detail - there is a small „step“ in it at $T \approx 304 K$ (Figure 3.4 - right hand side). It seems that such a heat capacity trace is a superimposition of a sharp component upon a considerably broader underlying component. Such an analysis of the heat capacity profile was applied in [39]. However, deconvolution of the heat capacity trace suggests that the system can be divided into one ,rich peptide“ component, which causes the broad profile, and one ,pure lipid“ component, which should give the same $C_{p}$-profile as obtained in the absence of proteins. The interaction between the two components is neglected. However, the last assumption seems not to be relevant, since the ,rich peptide“ component, which in our case is the fluid phase (Figure 3.6 - snapshots) and the gel domains have a permanent interface. Thus, this model calculation demonstrates that such a complicated heat capacity profile can be analyzed without assuming subcomponents in the system.

Let's now consider a peptide, which interacts unfavorably with both lipid phases but has preference to one of them, i.e. let's assume $\omega_{g p}>\omega_{f p}>0$. The heat capacity profile of such a mixture is given in Figure 3.7.

In this case the melting temperature of the system hardly changes. The heat capacity profile develops a shoulder at low temperatures. The MC simulation snapshots show that large peptide aggregates form in both the gel and the fluid lipid phase. But in the gel phase, the peptide aggregate is surrounded by lipids in the fluid phase. This ,neighborhood“ causes fluctuations in the membrane plane, which are the reason for the developed asymmetry in the $C_{p}$ - trace.

The latter case is very important, since it seems to be valid for gramicidin A incorporated in DPPC or DMPC membrane. 


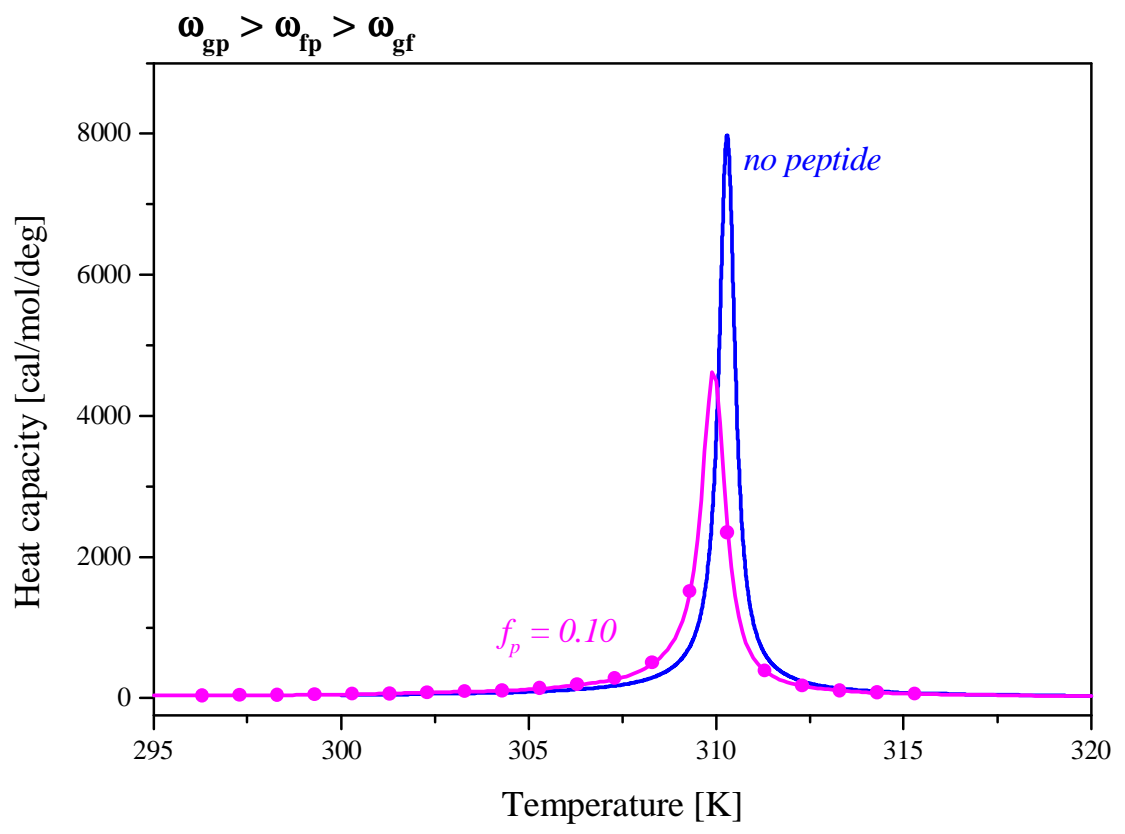

$$
\mathbf{T}=\mathbf{T}_{\mathrm{m}}-\mathbf{3}
$$

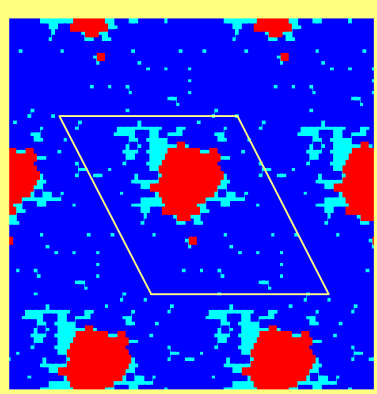

$\mathbf{T}=\mathbf{T}_{\mathrm{m}}$

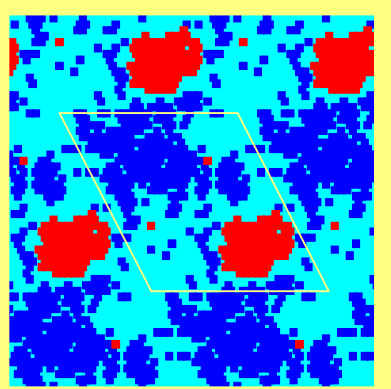

$$
\mathbf{T}=\mathbf{T}_{\mathrm{m}}+\mathbf{3}
$$

Figure 3.7. Top: Calculated heat capacity profile of a membrane containing $10 \mathrm{~mol} \%$ peptide which aggregates in both lipid phases, but has a preference to the fluid phase, $\omega_{f p}=600 \mathrm{cal} / \mathrm{mol}, \omega_{g p}=900 \mathrm{cal} / \mathrm{mol}, \omega_{g f}=315 \mathrm{cal} / \mathrm{mol}$. Lines: histogram results; blue: pure lipid system; pink: mixture. Solid circles: calculation from a two-component MC simulation. Bottom: Representative Monte Carlo snapshots of the lipid-peptide matrix at various temperatures. The periodicities stem from the periodic boundary conditions. 


\subsubsection{Intermediate cases}

The big advantage of the applied histogram method is that the heat capacity profiles of various lipid/peptide mixtures can be calculated quickly. In the following we will demonstrate some ,intermediate“ cases - we will investigate how the heat capacity profile changes when the interaction energies, $\omega_{g f}, \omega_{f p}$ and $\omega_{g p}$, are comparable to each other.

Heat capacity profiles of mixtures in which the interactions gel-peptide and fluid-peptide are more favorable than gel-fluid, are given in Figure 3.8. When interpreting these interaction parameters in terms of hydrophobic length, this means a situation where the hydrophobic length of the peptide is in between those of the gel and fluid lipids (Figure 3.8 - left-hand side).

It should, however, be noted that the comparison with the hydrophobic length is just a simple association. In fact, the parameters, $\omega_{g f}, \omega_{f p}$ and $\omega_{g p}$, are effective interaction energies (see Equation (3.2)) which generally include fluid-fluid, gel-gel and peptidepeptide interactions.

Here, we assume that either the peptide interacts equally with both lipid phases or matches better with the fluid phase (the opposite case can be calculated by analogy). Three cases are demonstrated, in which the peptide displays different solubility in the fluid phase (from top to bottom). It is evident that equal interactions between gel and peptide and fluid and peptide lead to a symmetric heat capacity profile (see the black curves in each case), which sharpens when the demixing between lipids and peptides increases. The phase transition temperature of the mixture decreases when $\omega_{g p}>\omega_{f p}$ and the absolute value of this shift seems to be dependent only on the difference $\left(\omega_{g p}-\omega_{f p}\right)$ (compare for example the blue lines). The increase of the last difference leads also to a broader shoulder on the low temperature side of the transition. 

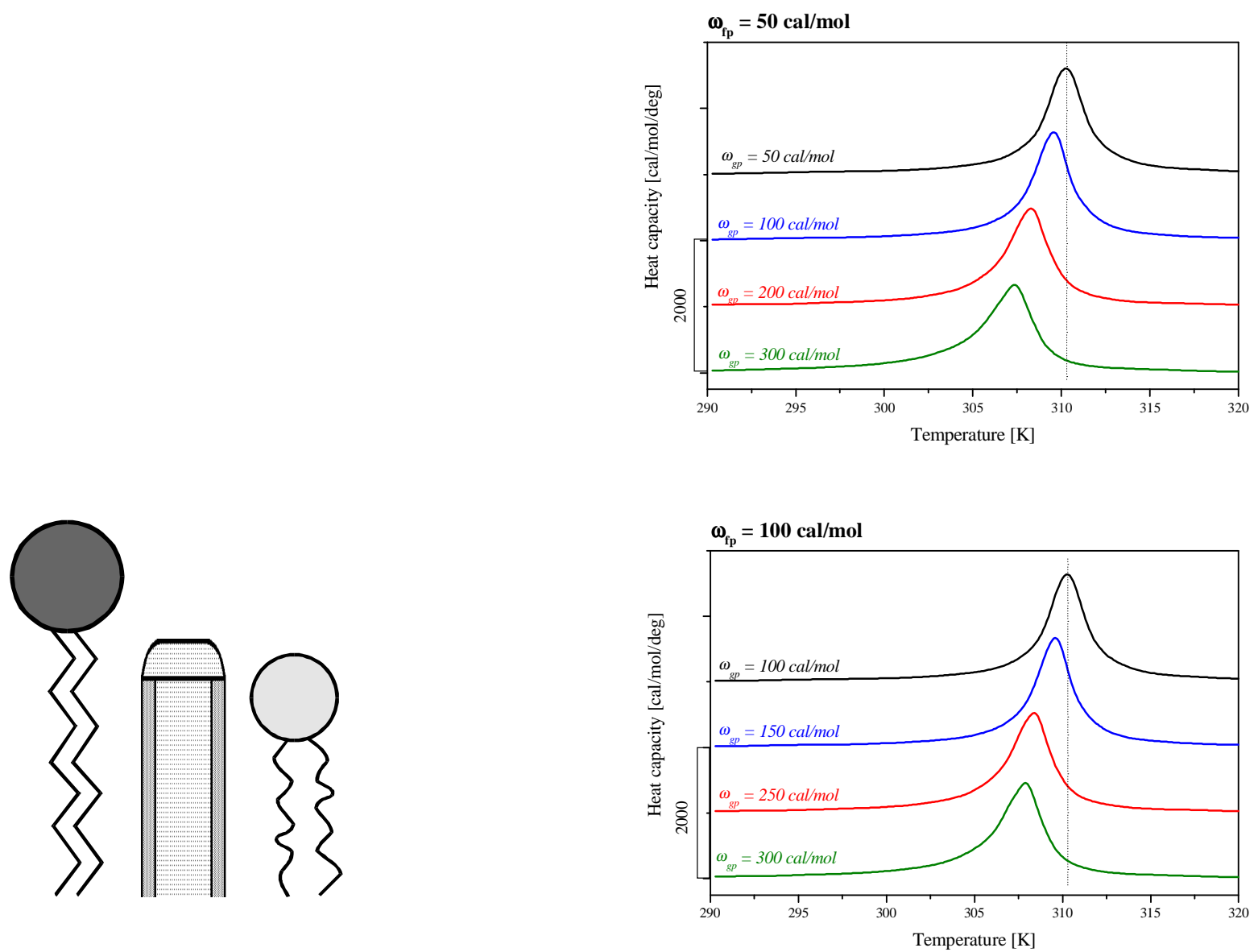

$$
\omega_{f p} \leq \omega_{g p}<\omega_{g f}
$$

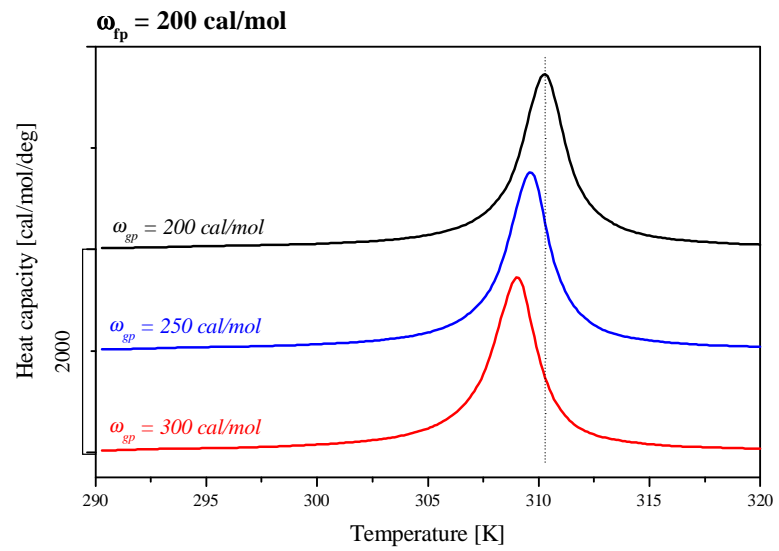

Figure 3.8. Heat capacity profiles, calculated from the histogram, for the case of a membrane containing $10 \mathrm{~mol} \%$ peptide that has a relatively weak tendency to aggregate in both lipid phases. The interactions between lipid and peptide are more favorable than between gel and fluid lipid, $\omega_{f p} \leq \omega_{g p}<\omega_{g f}=315 \mathrm{cal} / \mathrm{mol}$. The dotted vertical line shows the melting temperature of the pure lipid system. 

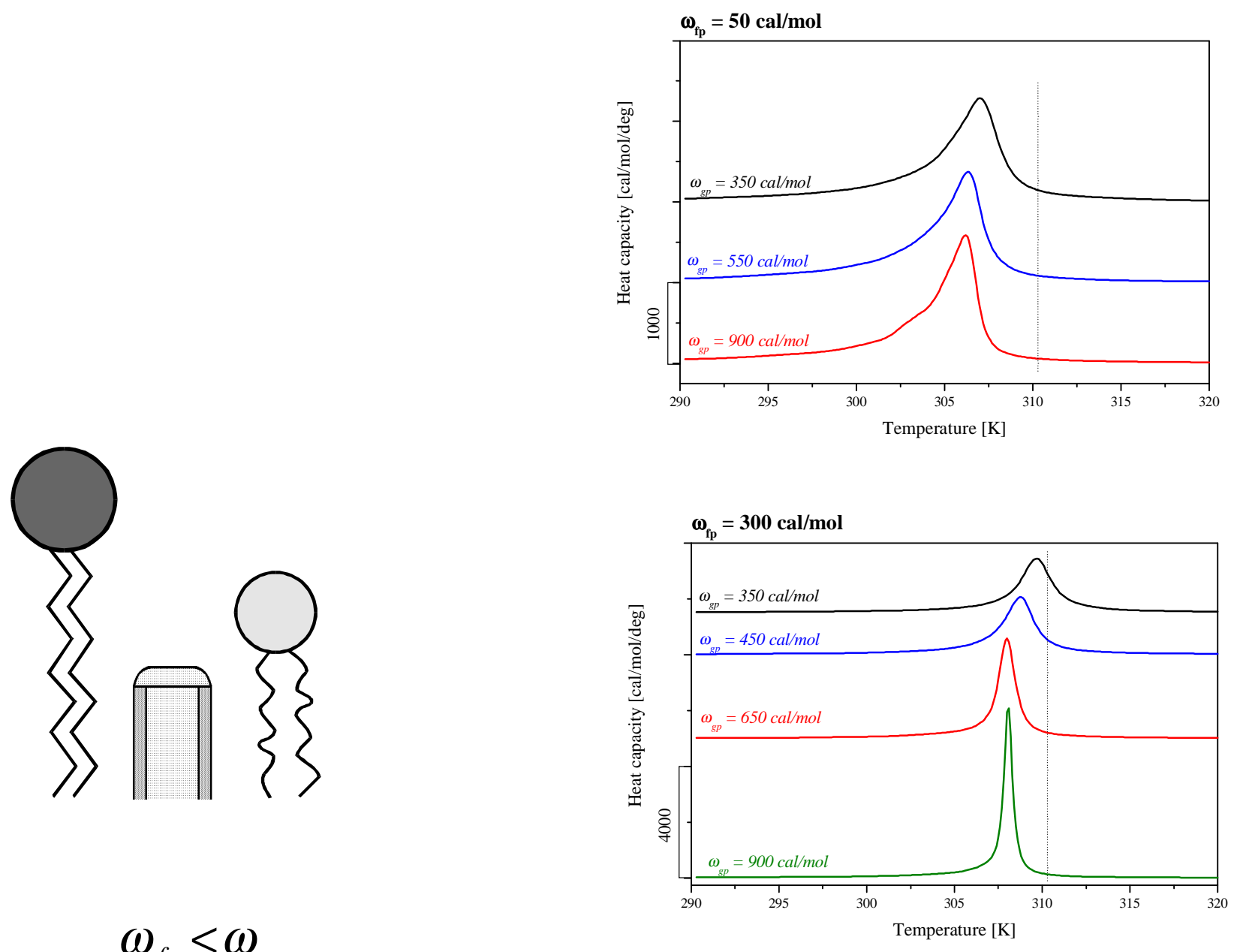

$$
\begin{gathered}
\omega_{f p}<\omega_{g p} \\
\omega_{g f}<\omega_{g p}
\end{gathered}
$$

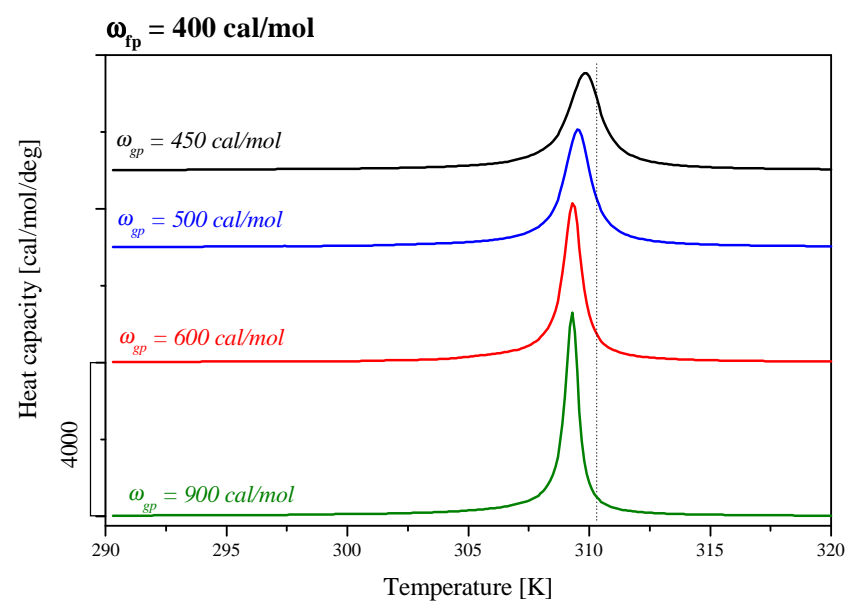

Figure 3.9. Heat capacity profiles, calculated from the histogram, for the case of a membrane containing $10 \mathrm{~mol} \%$ peptide that interacts preferentially with the fluid phase, $\omega_{f p}<\omega_{g p}, \omega_{g p}>\omega_{g f}=315 \mathrm{cal} / \mathrm{mol}$. The dotted vertical line shows the melting temperature of the pure lipid system. 
In the next example we would like to demonstrate the case when $\omega_{g p}>\omega_{f p}$ and $\omega_{g p}>\omega_{g f}$. Hence, the peptide dissolves better in the fluid phase than in the gel phase and the contacts gel-fluid are more favorable than the contacts between gel and peptide. The calculated heat capacity traces of such mixtures are given in Figure 3.9. In the case where the fluid-peptide interactions are either more favorable (top graph) or unfavorable (bottom graph) in comparison to the fluid-gel contacts, the shift of the melting transition to lower temperatures is almost independent from the degree of peptide aggregation in the gel phase. The situation is, however, more different where the two interaction parameters are comparable, $\omega_{f p} \sim \omega_{g f}$ (the middle graph). In this case, the temperature shift of the transition increases with increasing $\omega_{g p}$ up to a certain value (black, blue and red line) and then slightly decreases (olive line).

The model cases considered in this section give an overview of typical experimental situations. The heat capacity profiles presented here are helpful for a fast qualitative analysis of measured DSC traces of lipid-peptide mixtures.

\subsection{Comparison with experimental heat capacity profiles}

The experimental systems we investigated by differential scanning calorimetry were various mixtures of DPPC or DMPC membranes with relatively small peptides like alamethicin and gramicidin A.

The DSC-traces of DPPC/alamethicin mixtures given in Figure 3.10 develop long shoulders in the low temperature region and there is a small increase of the phase transition temperature by $0.3 \mathrm{deg}$. These heat capacity profiles suggest that the peptide aggregates in the gel DPPC phase (compare e.g. to Figure 3.4). However, we were not able to make a fit of these profiles, since in our simulations such a large asymmetry in the heat capacity profile is always connected to a depression of the melting temperature. This point will be discussed in more details in Section 3.9. 


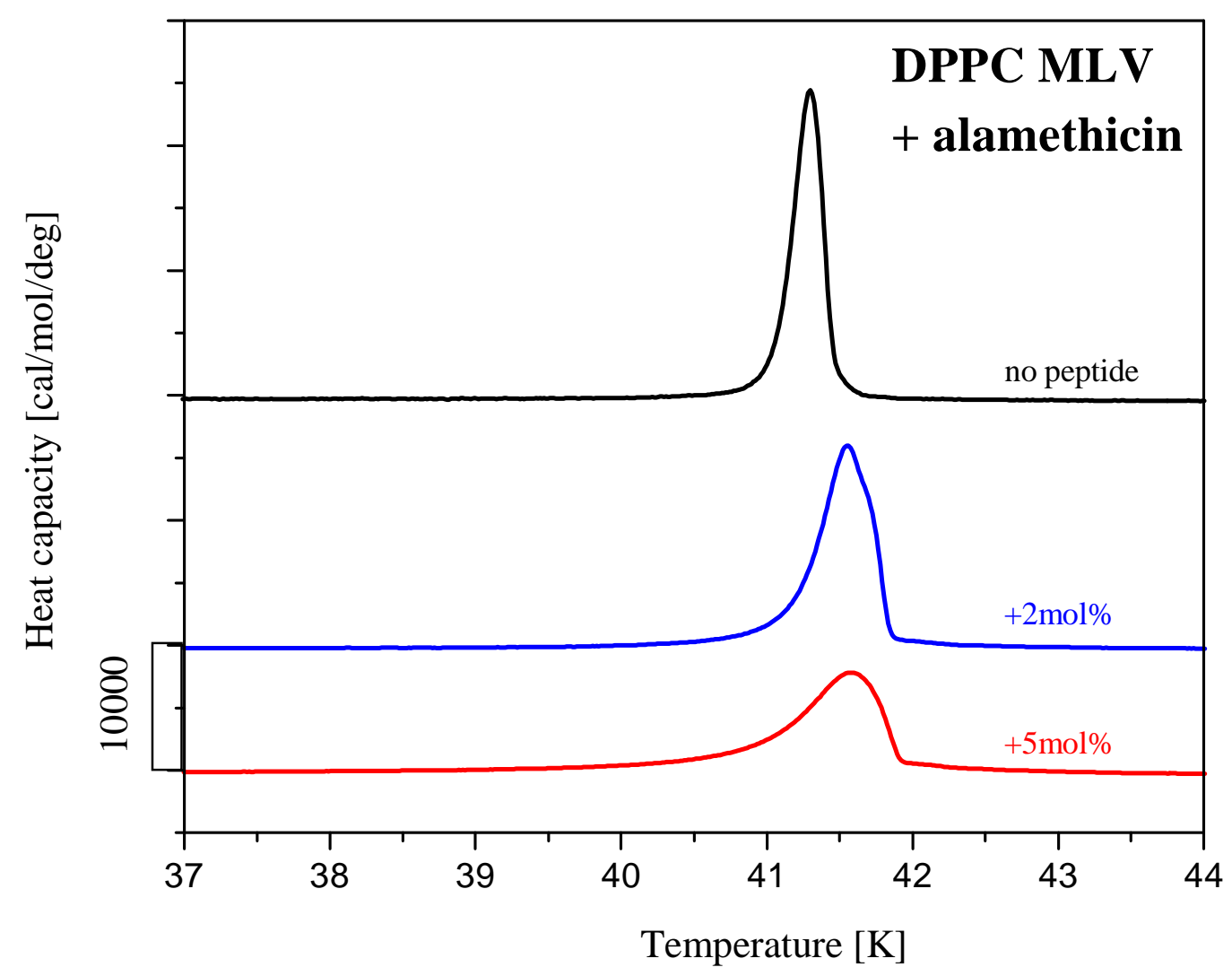

Figure 3.10. Calorimetric heat capacity traces of DPPC membranes containing various amounts of alamethicin. Multilamellar vesicle dispersions in buffer $(5 \mathrm{mM}$ Hepes, 1 mM EDTA, pH 7.5). Scan rate 5 deg/hour.

The other type of model systems investigated by means of differential scanning calorimetry were mixtures of the peptide antibiotic gramicidin A with DPPC and DMPC. Figure 3.11 shows the NMR structure of gramicidin A in a DPPC membrane. It is evident that the gramicidin A dimer is relatively short in comparison to the thickness of the DPPC bilayer. This suggests an unfavorable hydrophobic mismatch between the peptide and the lipids. The heat capacity traces of DPPC/gramicidin A and DMPC/gramicidin A mixtures are shown in Figure 3.12 and Figure 3.13, respectively. In the case of DPPC bilayer, the $C_{p^{-}}$ profiles of two vesicle preparations with different cooperativity of the melting transition are given. In all cases, the insertion of gramicidin A into the lipid bilayer induces an extended shoulder at the low temperature end of the gel-to-fluid transition. This asymmetry suggests a pronounced peptide aggregation in the gel lipid phase, whereas the demixing in the gel DPPC bilayer should be stronger than in the gel DMPC membrane. This is a plausible 
conclusion, since the gel DMPC membrane is by about $5 \AA$ thinner than the gel DPPC membrane and thus the hydrophobic mismatch between gramicidin $\mathrm{A}$ and the lipids is likely to be larger in the case of DPPC. Gramicidin A causes a very slight shift of the melting transition to lower temperatures in both types of lipids.

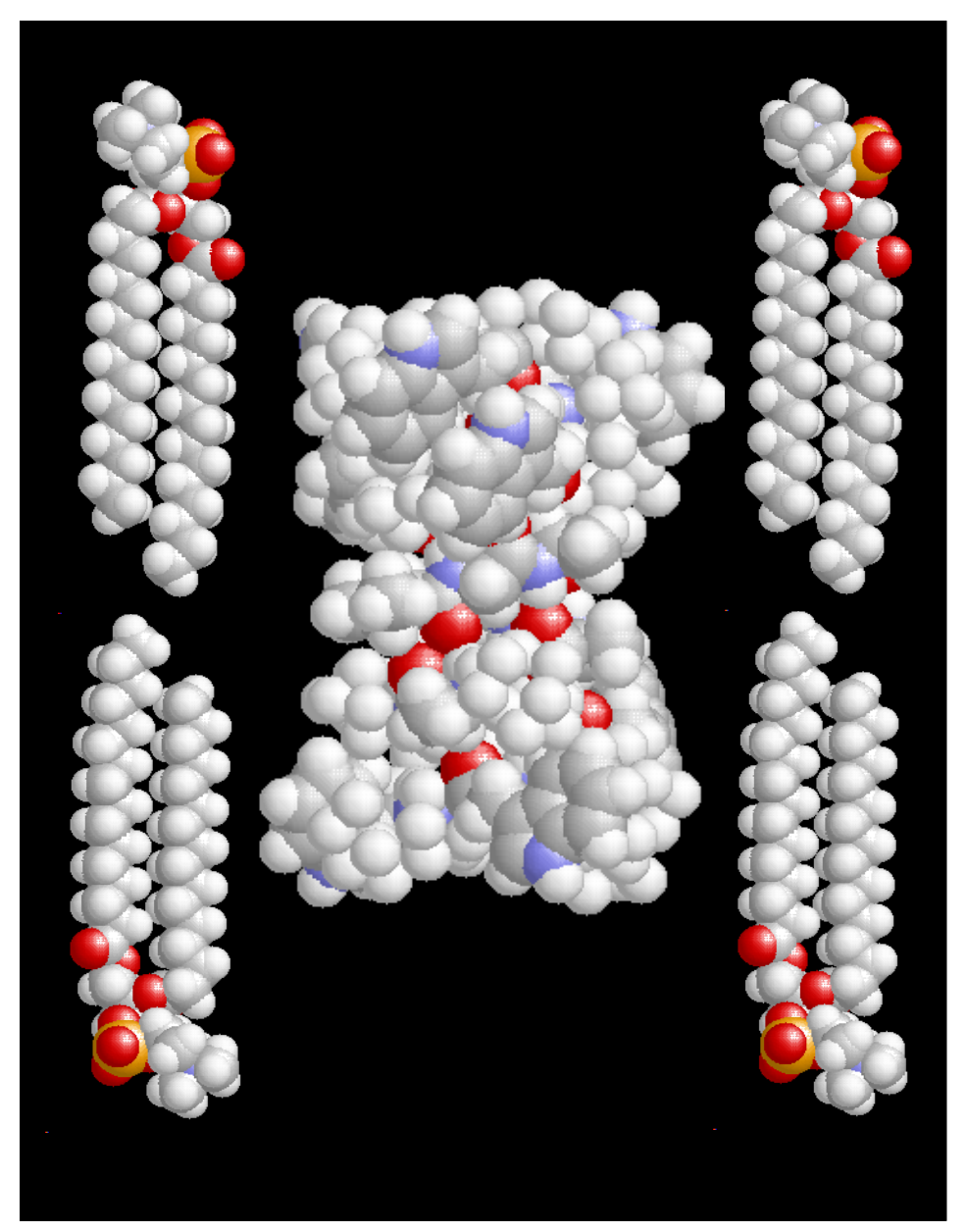

Figure 3.11. Gramicidin A (NMR) in a DPPC membrane. The gramicidin A dimer is relatively short, suggesting an unfavorable hydrophobic matching in both lipid phases. 

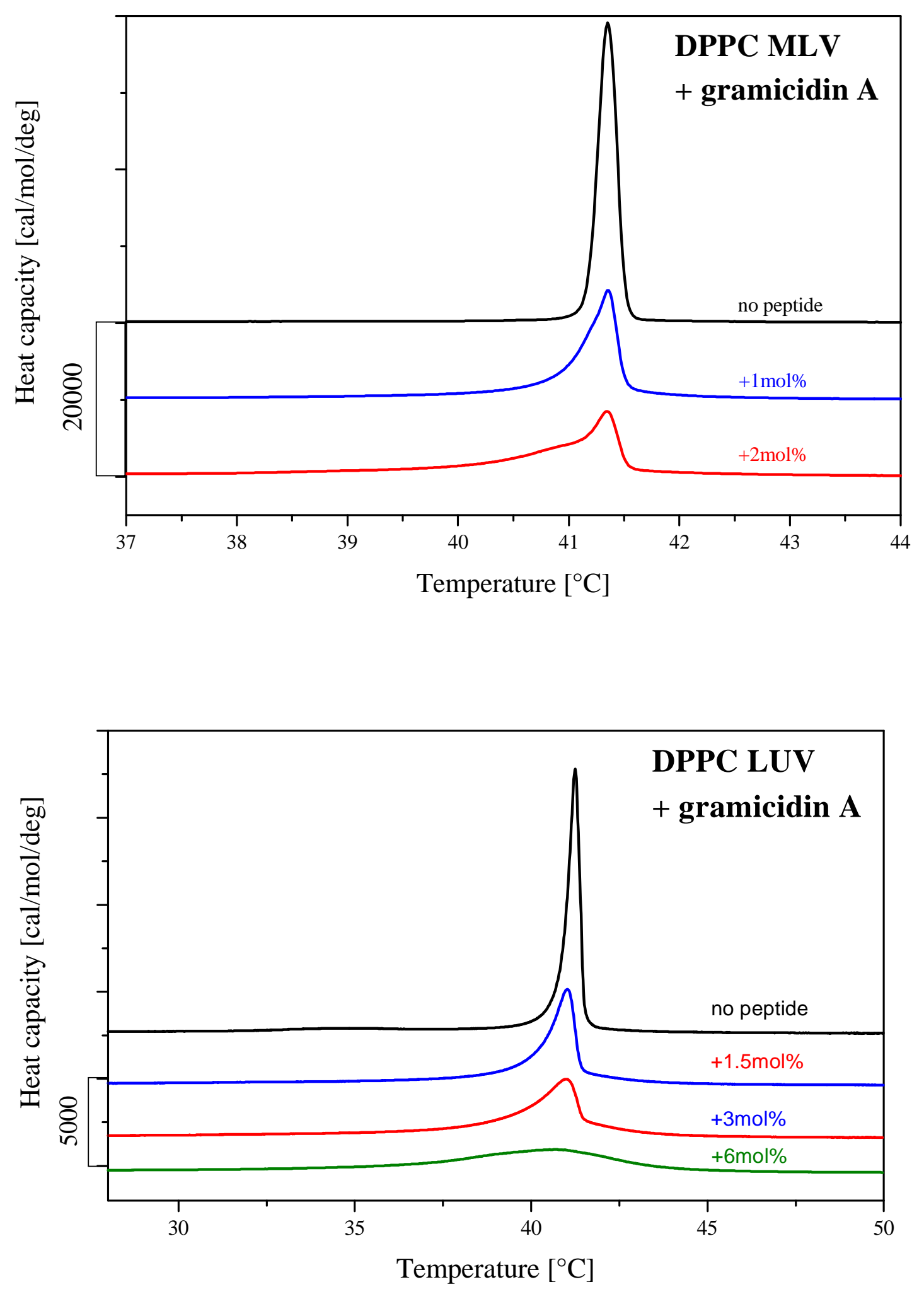

Figure 3.12. Calorimetric heat capacity traces of DPPC membranes containing various amounts of gramicidin A. Top: MLV. Bottom: LUV. Vesicle dispersions in buffer (5 mM Hepes, 1 mM EDTA, pH 7.5). Scan rate 5 deg/hour. 


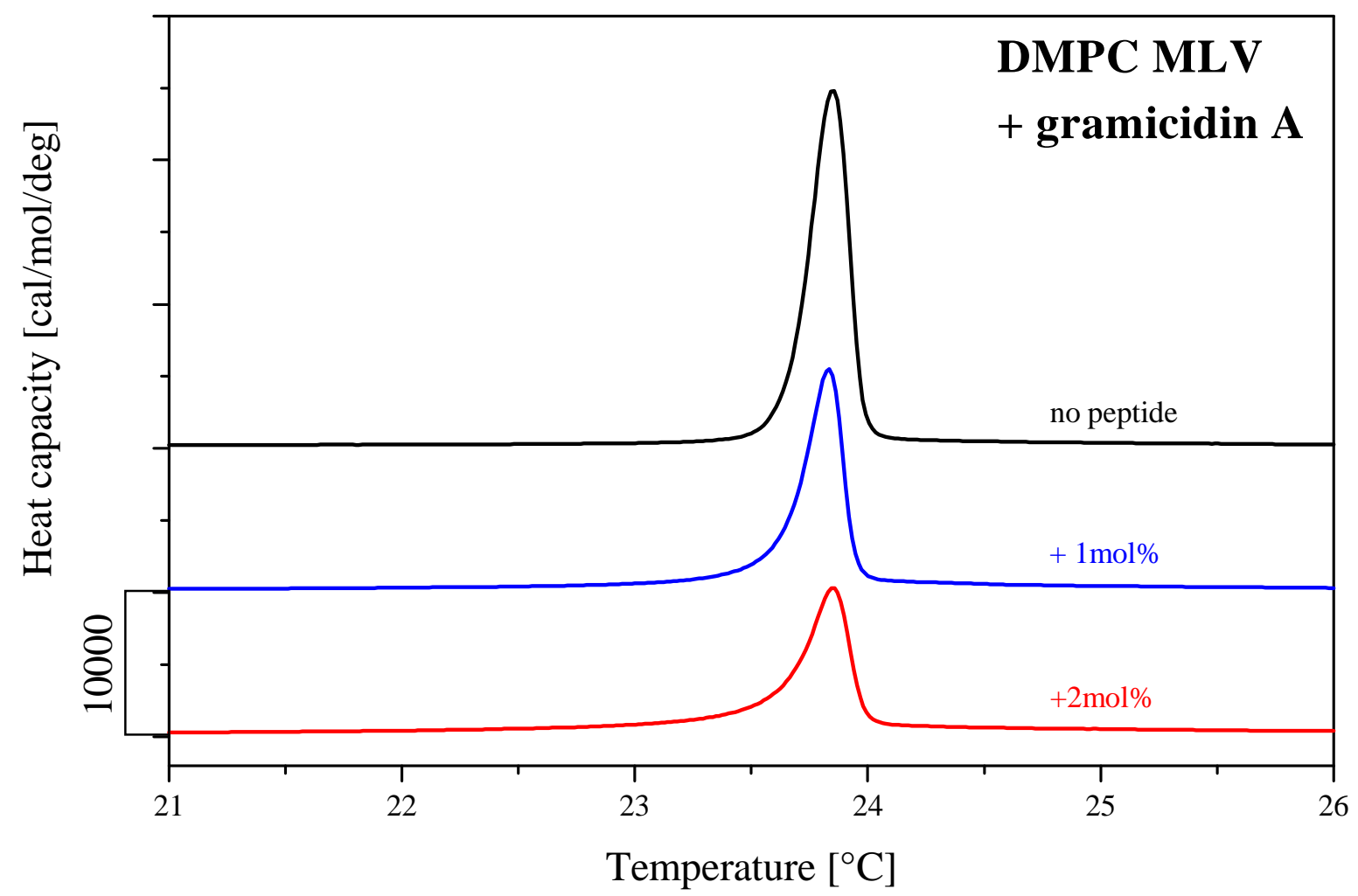

Figure 3.13. Calorimetric heat capacity traces of MLV dispersions of mixtures of DMPC with various amounts of gramicidin A. Vesicle dispersions in buffer $(5 \mathrm{mM}$ Hepes, 1 mM EDTA, pH 7.5). Scan rate 5 deg/hour.

A quantitative comparison between a DSC trace of DPPC membrane containing $2 \mathrm{~mol} \%$ gramicidin A and a heat capacity profile obtained from the histogram is given in Figure 3.14. In fact, the peptide gramicidin A is somewhat larger than a single DPPC lipid. Its cross-section has an area of about $180 \AA^{2}$ and that of the lipid is about $45 \AA^{2}$. This should be accounted in the MC simulations, i.e., one has to perform MC simulations on a mixed lipid-peptide system where the peptide occupies 4 lattice sites. It would not be correct to simply perform a calculation with 4-times larger peptide concentration, because in the two cases both the interfacial energy on the lipid-peptide boundary and the entropy are different. Four peptides while occupy the lattice sites corresponding to one large peptide still increase the entropy of the system by 4 !, because of the number of different arrangements. On the other hand, a membrane that contains smaller peptides has a larger lipid-peptide interface than the corresponding case of large peptides. However, when a pronounced protein aggregation takes place only the boundary peptides in the cluster are in 
contact with the lipids. This is likely to be the case for gramicidin A, because the heat capacity profiles of its mixtures with DPPC and DMPC are strongly asymmetrically broadened.

We neglected the entropic contribution resulting from the size of the single peptide and performed calculations at peptide fraction $f_{p} \approx 4 \cdot x_{p}$, where $x_{p}$ is the fraction of the protein in the experiment, expecting a similar trend.

The calculated profile shows similar properties as the measured one - a slight shift of the phase transition temperature and an asymmetry at the low-temperature end of the transition. But there are some significant differences between the two profiles. The possible reasons for these differences will be discussed in Section 3.9.

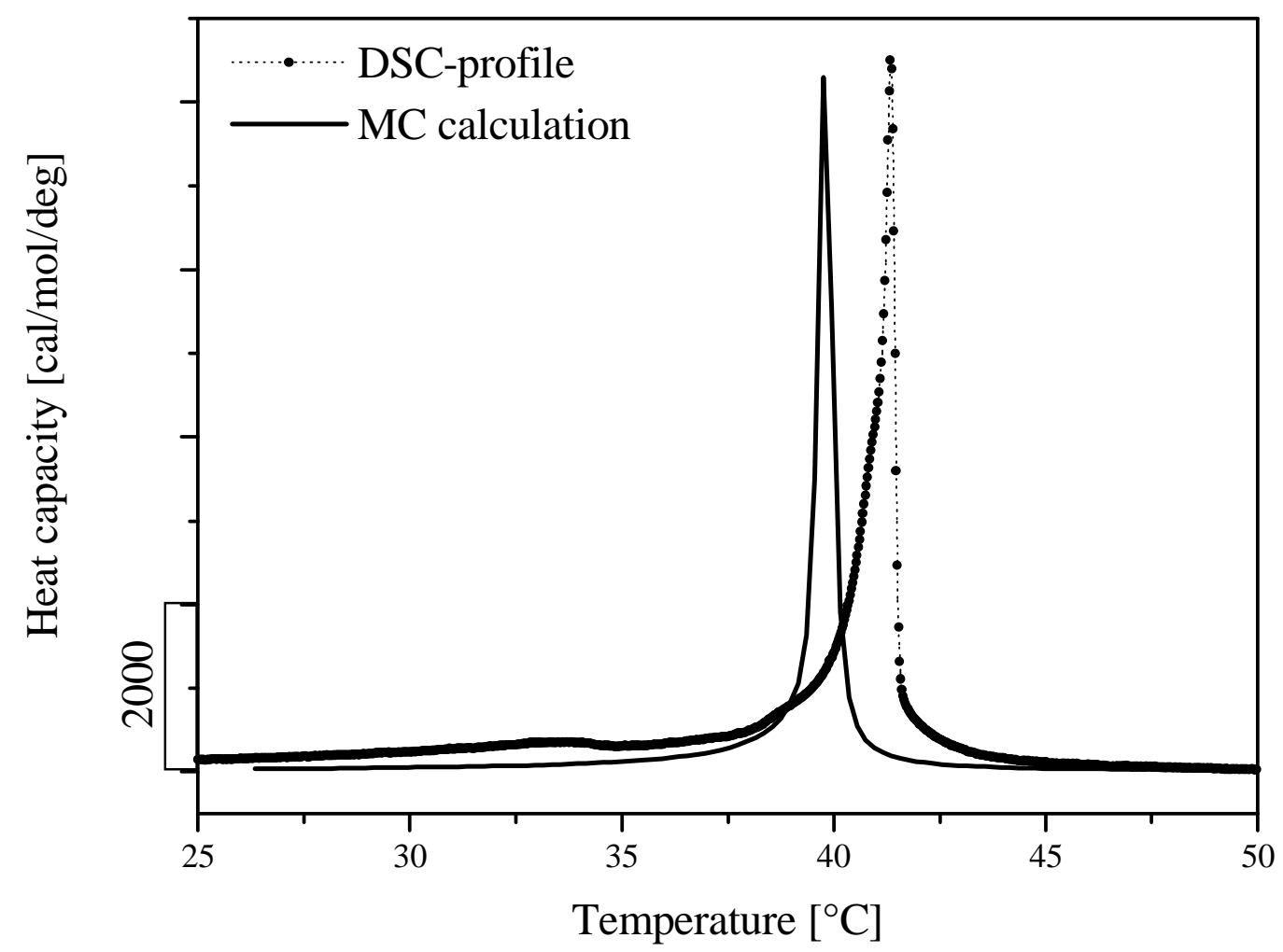

Figure 3.14. Comparison between measured DSC-trace of DPPC/gramicidin A mixture and calculated heat capacity profile. Solid circles: $C_{p^{-}}$profile of MLV dispersion of DPPC with incorporated 2 mol\% gramicidin A. Solid line: heat capacity profile, derived from the broad histogram. Parameters of the calculation: $f_{p}=0.1, \Delta H=8700 \mathrm{cal} / \mathrm{mol}, \quad T_{m}=41,35^{\circ} \mathrm{C}, \quad \omega_{g f}=320 \mathrm{cal} / \mathrm{mol}, \quad \omega_{f p}=350 \mathrm{cal} / \mathrm{mol}$ and $\omega_{g p}=900 \mathrm{cal} / \mathrm{mol}$. 
The parameters used for the calculation correspond to a case where the peptide interacts unfavorably with both lipid phases but its solubility in the fluid phase is better than in the gel phase. Therefore, we expected, on the basis of the shape of the heat capacity profiles, that there were large peptide aggregates in the low temperature phase and smaller ones at high temperatures. In order to test these predictions, we performed atomic force microscopy measurements, as described in the next section.

\subsection{Atomic force microscopy experiments}

Since the peptide gramicidin A forms dimers with a length of about $30 \AA$, thus shorter than the DPPC or DMPC bilayer (see Figure 3.11), it was possible using the AFM-technique to resolve the location of the peptide in the membrane plane. Additionally, the different melting temperatures of DPPC $\left(\sim 41^{\circ} \mathrm{C}\right)$ and DMPC $\left(\sim 24^{\circ} \mathrm{C}\right)$ allowed to perform measurements both in gel and in fluid phase at room temperature.

AFM-images of gel DPPC membrane with and without incorporated peptide gramicidin A are given in Figure 3.15 and 3.16, respectively. In the absence of peptide, supported DPPC bilayers have a flat appearance under AFM in solution. There are also some bilayer defects (dark areas). The thickness of the gel DPPC bilayer, which we measured from the edge of these defects, is about $7 \mathrm{~nm}$, which is in a good agreement with other values [22]. When 2 mol\% gramicidin A was incorporated into a DPPC bilayer, the surface remained largely planar, but we observed line type depressions of about $0.5 \mathrm{~nm}$ depth sparsely distributed in the membrane segment (Figure 3.16). Similar line-shaped defects in gel state DPPC membranes were found in AFM experiments by Mou et al. [22]. In the case of fluid DMPC membrane with incorporated $2 \mathrm{~mol} \%$ gramicidin A (Figure 3.17) we observed again such depressions, but they were of a round shape.

In both cases, the depressions in the membrane were identified as peptide clusters. We believe that the different type of protein aggregates in the gel and fluid membrane is caused by the following: There is an experimental evidence that in the gel phase the lipid molecules form a hexagonal lattice [15]. Therefore, the total demixing of gramicidin A molecules from the gel DPPC phase results in forming large line-type defects in this lattice. We suppose this to be the reason for observing line-shaped peptide aggregates. On the other hand, in the fluid phase there is no lattice arrangement of the lipid molecules [15], 
leading to the presence of round-shaped peptide clusters, since in such a configuration the peptide-lipid interface is minimal.
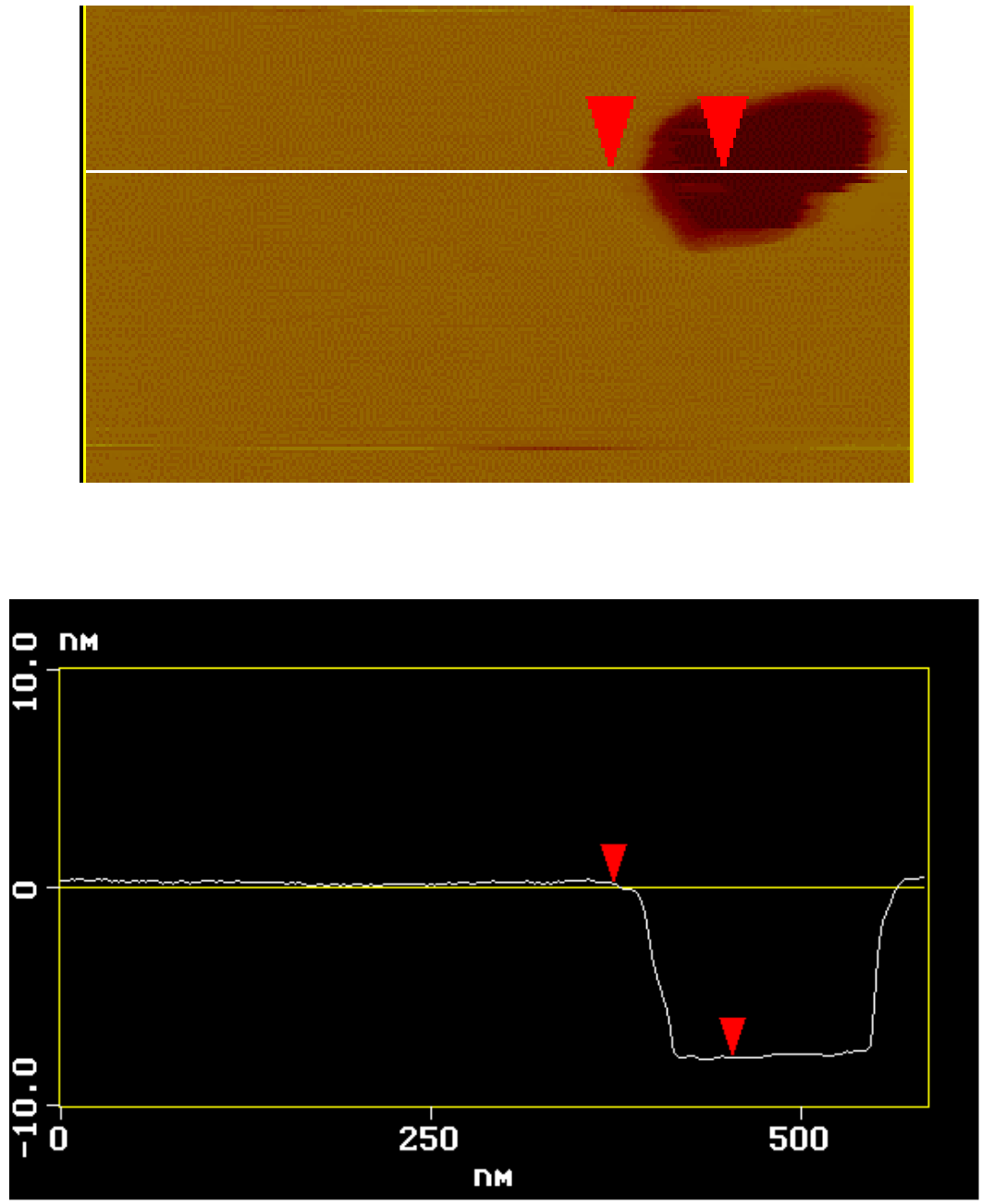

Figure 3.15. DPPC membrane in the absence of gramicidin A. Occasionally occurring defects display a thickness which corresponds roughly to the thickness of the membrane. Top: Original image. Bottom: Height profile across a defect. 


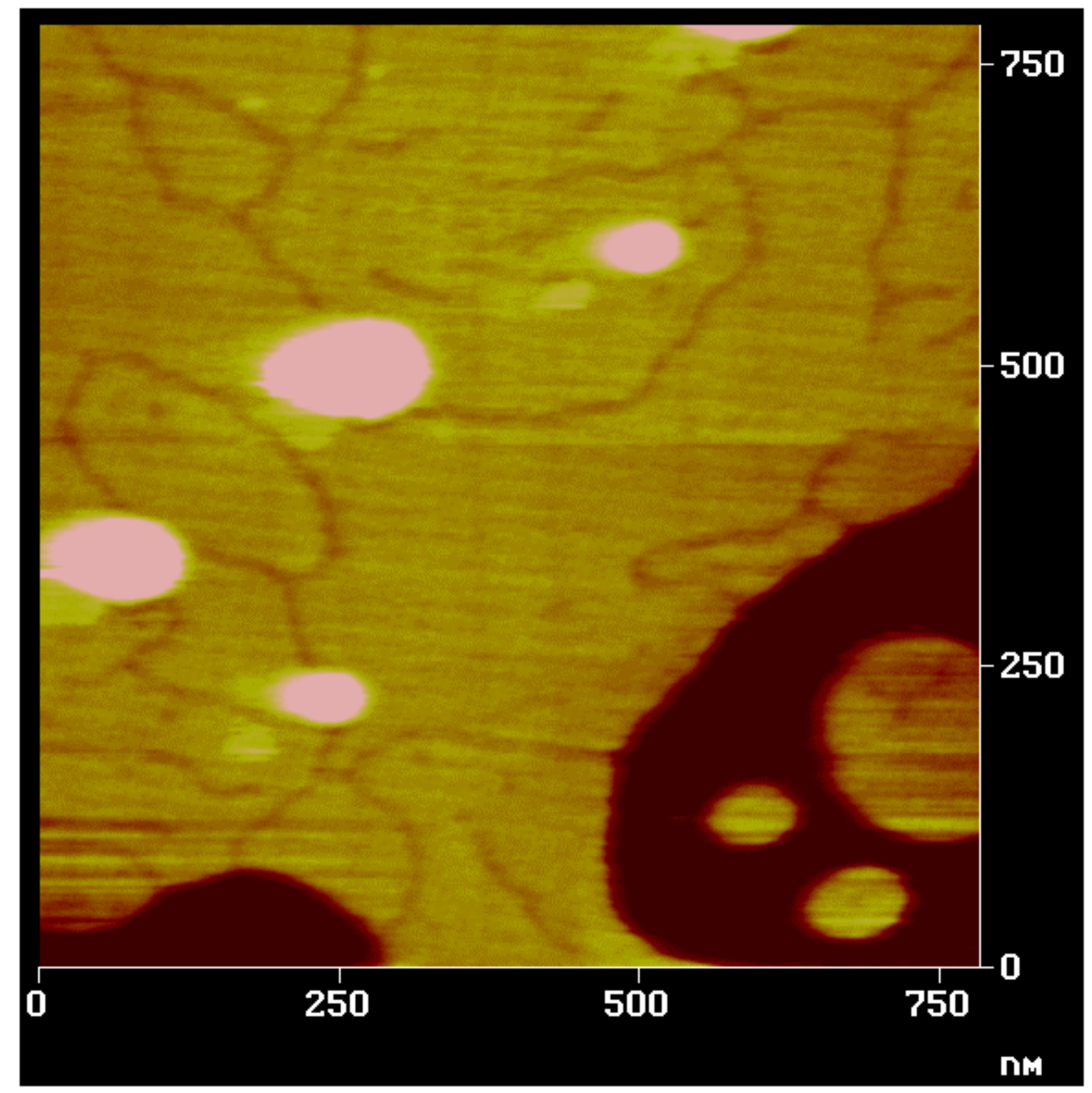

Figure 3.16. DPPC membrane in the presence of 2 mol\% gramicidin A. The light areas display bilayer segments on the mica surface. The peptide is accumulated in line shaped defects within the membrane plane. Note that the angle between crossing line depressions is about $120^{\circ}$, which agrees with the assumption that the peptide aggregates are defects in the hexagonal lipid lattice. 

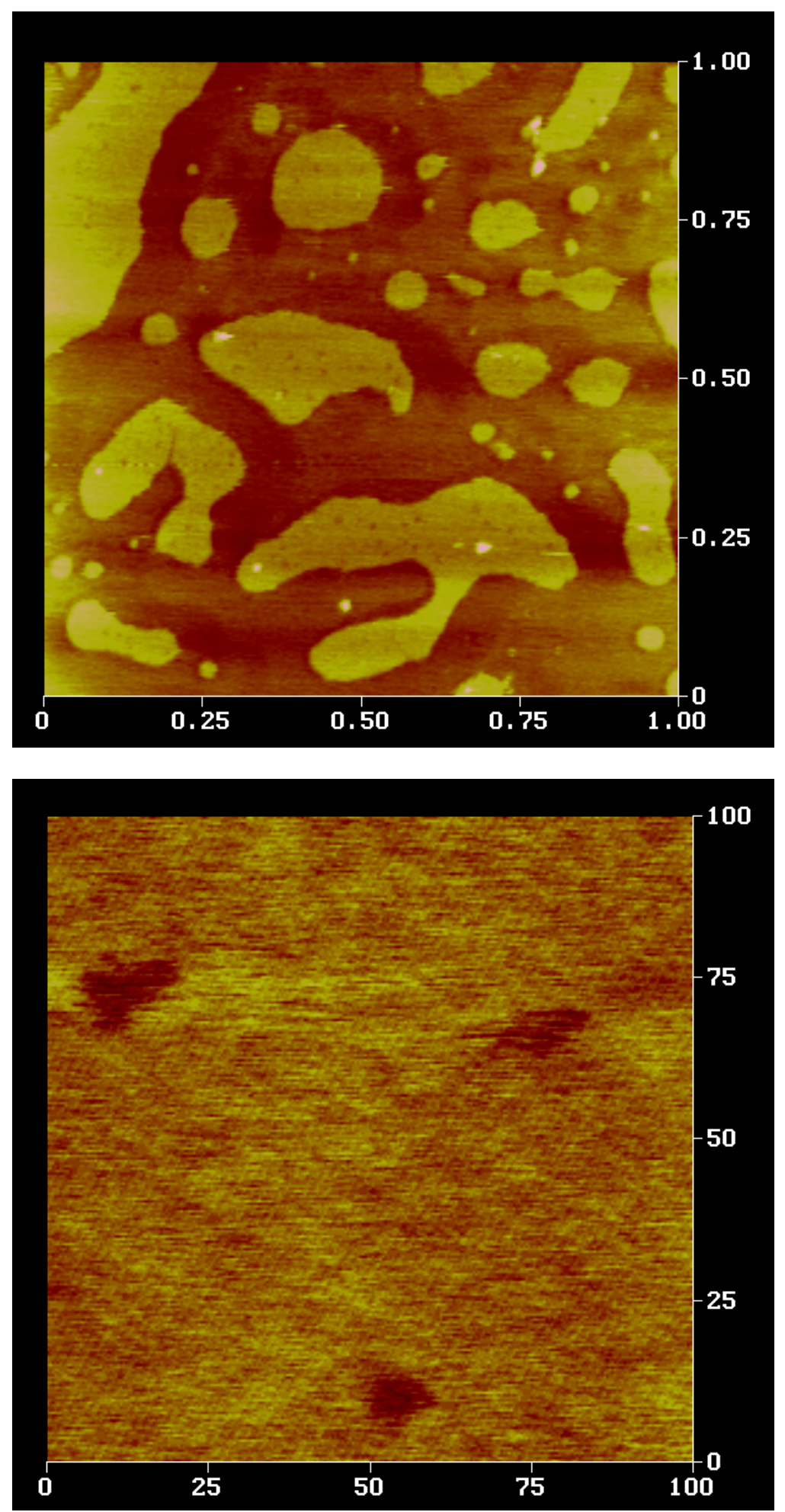

Figure 3.17. DMPC membrane in the presence of $2 \mathrm{~mol} \%$ gramicidin $\mathrm{A}$ in two different magnifications. Gramicidin A is accumulated in round defects (100-200 diameter) within the membrane plane. Top: Scanned area $1 \times 1 \mu \mathrm{m}$. Bottom: Scanned area $100 \times 100 \mathrm{~nm}$. 
However, the gramicidin A aggregation in the fluid membrane is not as pronounced as in the case of gel bilayer. The round clusters have a diameter in the range of $10 \mathrm{~nm}$ corresponding to about 40 protein molecules. In the gel phase we observed no small aggregates - all the peptide molecules seem to be concentrated in the line defects, thus totally demixing from the gel membrane.

Therefore, we conclude that there is a good agreement between the predictions, based only on the heat capacity profiles of gramicidin A mixtures with DPPC and DMPC, and the results from the AFM experiments.

\subsection{Peptide-induced membrane fluctuations}

With interaction parameters corresponding to the case of peptides that aggregate in both lipid phases but have a preference to the fluid phase (like gramicidin A in DPPC membranes), we performed Monte Carlo simulations in order to obtain information about the distribution of the fluctuations in the bilayer plane. During the simulations we sampled the value

$$
\bar{c}_{p}(x, y)=\Delta H \cdot \frac{\left\langle\text { LipidState }(x, y)^{2}\right\rangle-\langle\operatorname{LipidState}(x, y)\rangle^{2}}{R \cdot T^{2}}
$$

which can be interpreted as a heat capacity at lattice site $(x, y)$. Here the brackets \langle\rangle denote time averaging, $\Delta H$ is the increase of the enthalpy upon melting of a single lipid, $T$ is the temperature and LipidState $(x, y)$ is a function describing the state of the lipid at lattice site $(x, y)$; LipidState $(x, y)=1$ for fluid, LipidState $(x, y)=-1$ for gel. The peptides do not move during sampling. Since there is a very pronounced peptide aggregation in both lipid phases at the chosen set of parameters we assume that the time required for peptide diffusion is much larger than the characteristic time of the lipid fluctuations. The lateral distribution of the fluctuations in the membrane plane for this case is illustrated in Figure 3.18 . 


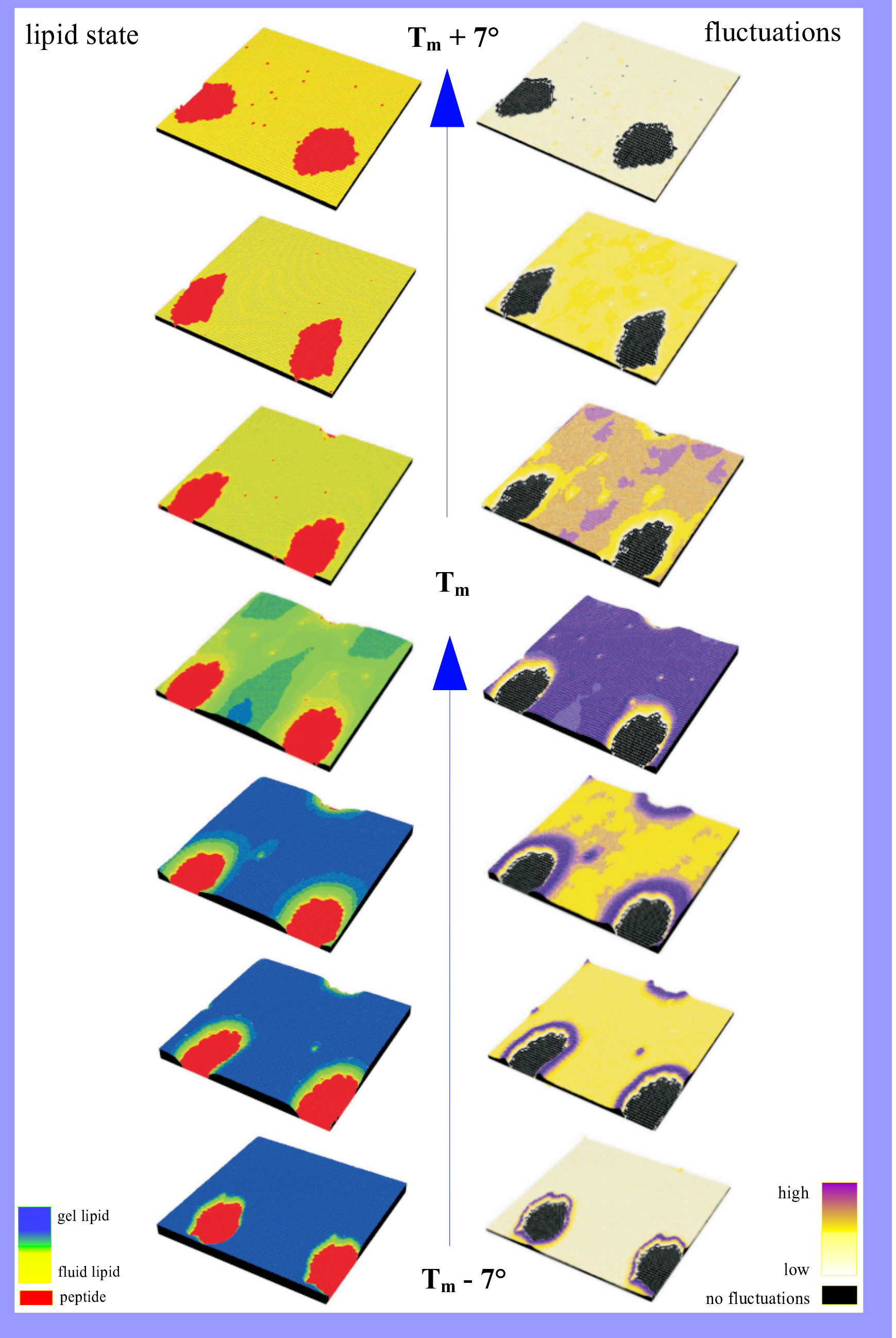


Figure 3.18. Spatial distribution of the fluctuations in a membrane containing 10 mol\% peptides that aggregate in both lipid phases, but have a preference to the fluid phase. Left: Averaged lipid state, $\langle$ LipidState $(x, y)\rangle$; Right: Amplitude of the thermal fluctuations, $\bar{c}_{p}(x, y)$. Simulations on a 61.61 matrix with periodic boundary conditions with the following interaction parameters: $\omega_{g f}=330 \mathrm{cal} / \mathrm{mol}$, $\omega_{f p}=660 \mathrm{cal} / \mathrm{mol}, \omega_{g p}=990 \mathrm{cal} / \mathrm{mol}$.

In the illustrated case the different peptide solubility in the gel and the fluid phase induces membrane fluctuations which are spatially non-homogenous. The largest fluctuations are located at the lipid-peptide interface and their maximum amplitude is at the melting transition of the mixed system.

In an experiment one determines the heat capacity, which is a measure of the enthalpy fluctuations, as a macro-observable. These model calculations give insight into the spatial distribution of the fluctuations and thus allow a kind of interpretation of the heat capacity as a micro-quantity.

\subsection{Discussion}

The interaction of lipid membranes with integral proteins has been investigated theoretically by various groups. Based on the 10-state lipid model of Pink and Chapman [29] and Pink et al. [30], lipid-protein interactions were modeled assuming parameters related to attractive van der Waals forces between the lipids and the proteins and repulsive forces due to hydrophobic mismatch [24,33-35,5]. This results in a net interfacial free energy difference between the lipids and the proteins and suggests the possibility of aggregation of integral proteins in the absence of protein-protein interactions. If the length of the hydrophobic core of the protein differs from the lipid chain length, the interaction between the two components is unfavorable and they do not mix well. Experimental studies where the lipid chain length was altered showed that the effect of peptides on the melting reaction varies significantly [39]. In the melting transition the thickness of bilayers decreases by about 16\% [11], hence the hydrophobic thickness of the membrane differs between gel and fluid. Therefore, it is likely that the interaction of the proteins with the two lipid phases is not the same and that good mixing in one phase implies unfavorable mixing 
in the other state. The corresponding phase diagrams have been discussed theoretically in $[40,28]$.

The change in protein distribution has pronounced effects on the lipid melting behavior [12]. The heat capacity traces are shifted and asymmetrically broadened. From the shape of the melting curves one can in principle deduce how the components mix in either phase. For suitable chain lengths of the lipids there are proteins that mix well with one membrane state and do not mix with the other membrane state [39].

We applied the two-state Ising model to describe the melting behavior of a lipid membrane with incorporated small integral protein. The relatively low number of variables, needed to describe the system energy (number of fluid lipids, $f$, and the number of three types of unlike nearest neighbor contacts, $n_{g f}, n_{f p}, n_{g p}$ ), allows construction of a broad histogram of states. This histogram can be used to calculate quickly mean values of various physical observables (which are functions of $f, n_{g f}, n_{f p}$ and $n_{g p}$ ) of the mixed lipid-peptide system.

Using the monolayer histogram technique for a two-state Ising model we calculated the heat capacity profiles of several lipid-peptide mixtures (Figure 3.3-3.9). The results show that the specific asymmetry of the heat capacity profiles can be non-ambiguously interpreted as peptide aggregation in the respective lipid phase.

The simple case of a membrane containing peptides that mix well with the gel state and demix in the fluid state (Figure 3.3) can even be resolved using the information sampled in the two-dimensional histogram for a pure lipid bilayer in the absence of peptides (Equation (3.5)). Depending on the peptide concentration, the heat capacity profiles of such mixed systems are progressively shifted to higher temperatures and asymmetrically broadened with a shoulder at the high temperature end. The case of gel-like peptide has previously been described theoretically by [12] but for larger proteins (occupying 19 lattice places) and without use of the histogram technique. An experimental example for such a case is the mixing of the band 3 protein of erytrocytes with phosphatidylcholine membranes [21]. For lipid chain length where the peptides mix well with the fluid phase the situation is the opposite: $C_{p}$ profiles are shifted to lower temperatures with a shoulder on the low temperature site (Figure 3.4). Similar heat capacity traces are measured on DMPG bilayers with incorporated gramicidin S [31] (Figure 3.19). The comparison between Figure 3.19 
and Figure 3.4 leads to the conclusion that gramicidin S aggregates in the gel DMPG phase but is homogeneously distributed in the fluid phase. However, there is by far no experimental proof of this theoretical prediction.

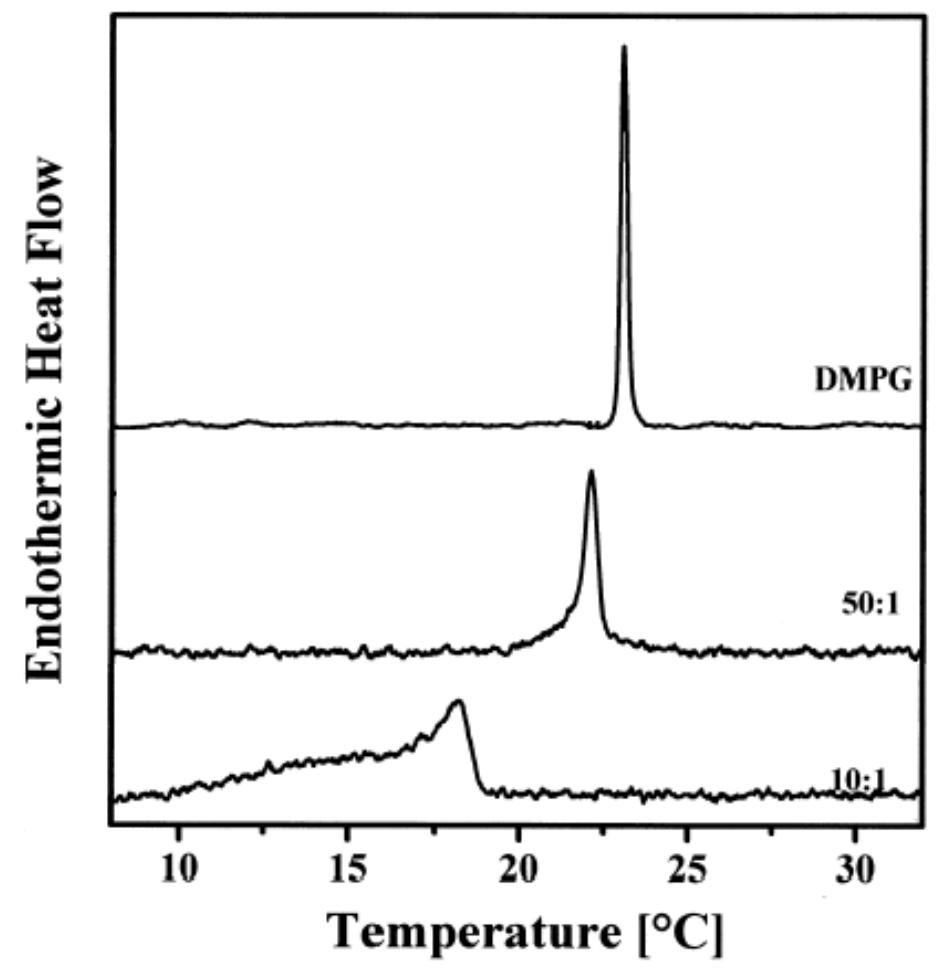

Figure 3.19. DSC heating scans illustrating the effect of the addition of gramicidin $\mathrm{S}$ on the main phase transition of 1,2-dimirystoyl-sn-glycero-3-[phospho-rac-(1glycerol)] (DMPG) multilamellar vesicles. The numbers above each curve denote the lipid-to-peptide molar ratios. The vesicle dispersions were prepared in buffer (10 $\mathrm{mM}$ Tris- $\mathrm{HCl}, 100 \mathrm{mM} \mathrm{NaCl}$ and $2 \mathrm{mM}$ EDTA). (Adapted from [31]).

The histogram technique for use of MC simulation data is a very helpful approach in investigating the melting behavior of mixed lipid-peptide systems. Since in the case of such mixtures three interaction parameters, $\left(\omega_{g f}, \omega_{p}, \omega_{g p}\right)$, can be varied in order to generate different experimental situations, the application of the histogram method saves a lot of computer time. For instance, to get a good heat capacity profile one needs about 30 points (see e.g. a profile with a long shoulder in Figure 3.6); each of these points needs about 12 min. (10000 MC cycles on $31 \cdot 31$ matrix, Pentium III $700 \mathrm{MHz}$ ), resulting in a computer time required of 6 hours. The same profile can be calculated from the broad fourdimensional histogram within minutes. Applying the histogram technique one can even 
calculate heat capacity traces of a system with temperature-dependent interaction parameters, $\left(\omega_{g f}(T), \omega_{f p}(T), \omega_{g p}(T)\right)$.

The hydrophobic interaction, which is an important part of the entire lipid-protein interaction, is temperature dependent [36]. Moreover, there is some experimental evidence [20] that in some cases peptides change their conformations to adapt to changes in bilayer thickness. Since the bilayer thickness changes upon the melting transition, i.e., is a function of the temperature, it would be reasonable to consider temperature-dependent interaction parameters.

The histogram method as applied here has, however, some disadvantages. It is possible to account only for small peptides, occupying only one lattice place. In fact, the peptides in the experimental systems are often larger than the lipids, which is a general problem in lattice models. The larger the peptides the larger their interface with the lipid neighbors in the lattice. This effect however can be accounted by adjusting the interaction energies, $\omega_{g p}$ and $\omega_{f p}$. But the distributional entropy of the peptides is smaller when the protein crosssection is larger. Therefore, there may be a grater tendency to form aggregates for larger proteins, because their loss of entropy upon aggregating is smaller than that of small peptides.

Performing a quantitative comparison between DSC profiles and calculated heat capacity traces, one should consider the following two effects:

1. Peptide size: cluster formation in the case of larger peptides, i.e., occupying more than one lattice site, becomes more pronounced (entropy effect), which results in a larger asymmetrical broadening of the heat capacity trace.

2. Matrix size: limits the size of the peptide cluster (naturally, the largest peptide aggregate contains no more than $n_{p}$ peptides). Hence, the peptides influence the membrane thermotropic behavior stronger in smaller systems, because the ratio lipid-peptide interface/system volume decreases with increasing the matrix size. This would decrease the effect of the temperature shift of the phase transition, which normally accompanies the peptide aggregation. 
Thus, we conclude that in the presented quantitative comparison between the DSC trace of a DPPC membrane containing gramicidin A and the histogram results (Figure 3.14), the calculations show a larger suppression of the transition temperature than it was actually detected in the experiment, which may partially be due to matrix-size effects but also to the fact, that the protein may not fit well into the lipid matrix. On the other hand, the lowtemperature shoulder of the DSC profile is more pronounced than in the calculated trace, because of effects related to the peptide size.

We applied the constructed broad histogram to a monolayer with incorporated $10 \mathrm{~mol} \%$ peptides to calculate the melting profiles of various systems. The interplay between the three interaction parameters produces very different shapes of the heat capacity profiles. From the simulation results it becomes evident, that an extended shoulder with a certain detail - the small step - represents very strong peptide aggregation in the respective lipid phase (see e.g. Figure 3.6 and also Figure 3.13 top, 2 mol\% gramicidin A).

The extremely pronounced aggregation of the peptide gramicidin A in the DPPC gel phase, predicted from the shape of the heat capacity trace, was confirmed by AFM experiments. $\mathrm{X}$-ray crystallography indicates that lipids are packed into a triangular lattice in the gel state, whereas they are disordered in the fluid $\mathrm{L}_{\alpha}$ phase [15]. Therefore, the line shaped gramicidin A aggregates observed in the low-temperature DPPC phase are supposed to be defects in the lipid lattice.

As the phase transition temperature of DPPC and DMPC membranes containing gramicidin A hardly changes, we concluded that gramicidin A aggregates also in the fluid phase. Round-shaped peptide aggregates in the fluid DMPC phase were detected with AFM. Since there is no lattice order in the fluid phase, the peptide clusters are round-shaped in order to minimize the interface with the lipid surrounding.

With the simple model proposed here it is impossible to explain many details of an experimental thermogram, for instance, the measured heat capacity profiles of DPPC/alamethicin mixtures which develop extended shoulders in the low temperature end of the transition, but are slightly shifted to higher temperatures. It is known that alamethicin molecules form a voltage-gated ion channel, which consists of 6-11 peptide molecules $[1,8,38,37,18]$. It was suggested that peptide aggregate formation is caused by lateral diffusion and electrostatic interaction in an antiparallel orientation. Generally, peptide- 
peptide interactions are included in our model (Equation 3.2). However, in the case of alamethicin there could be a specific type of interactions or conformational changes which we do not consider using only the effective interaction parameters, $\omega_{f p}$ and $\omega_{g p}$. On the other hand, the very extended shoulder in the heat capacity trace suggests a strong peptide clustering in the DPPC gel phase. There are experimental results which seems to be consistent with our interpretation of the DSC-trace. Figure 3.20 shows the temperature dependence of the lifetime of alamethicin ion channels in DPPC membranes. Whatever the reason for opening and closing of this ion channel, a necessary condition for the membrane conductance is the presence of peptide aggregates. The graph demonstrates that the channel lifetime decreases when approaching the phase transition temperature of the DPPC bilayer $\left(\sim 41^{\circ} \mathrm{C}\right)$. Many details of this process are still not clear. However, one possible interpretation of the observed channel behavior is that simply the degree of peptide aggregation decreases with the temperature approaching the phase transition temperature of the DPPC bilayer.

\section{opening time}

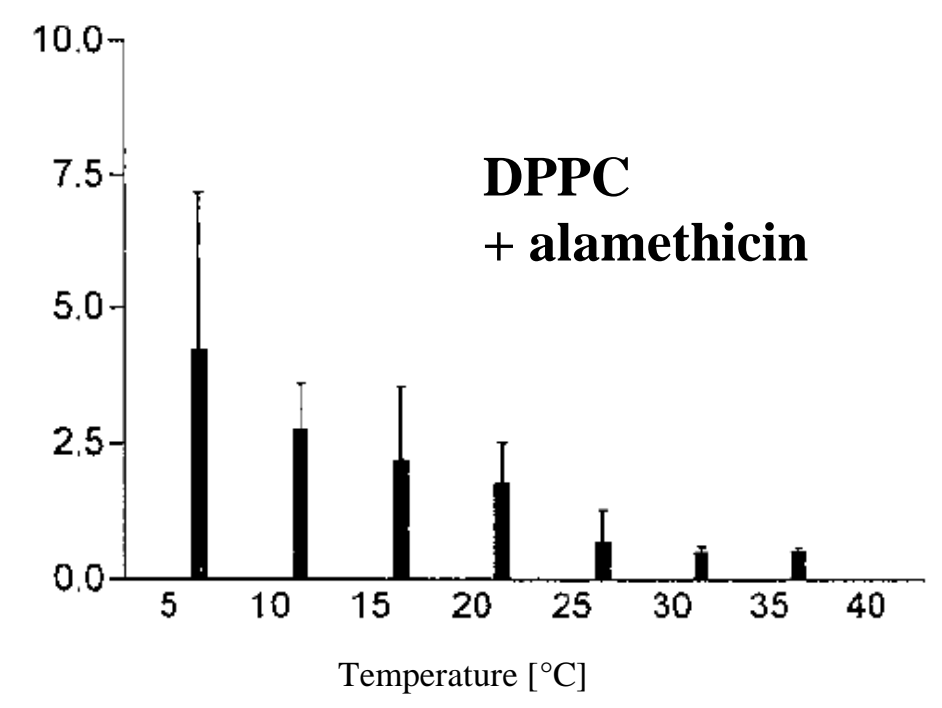

Figure 3.20. Opening time (msec) of alamethicin ion channels in planar DPPC bilayer at different temperatures. (Addapted from [16]).

For a DPPC membrane incorporating peptides that are well soluble in the fluid phase but demixes strongly from the gel phase we calculated a cluster formation parameter (defined as the mean number of peptide-peptide contacts per lattice site, i.e. $\left.\bar{n}_{p p}=n_{p p} / n\right)$. The change of the cluster parameter with temperature is shown in Figure 3.21. It is evident that 
its temperature dependence is very similar to that of the measured opening time of the alamethicin channel (Figure 3.20).

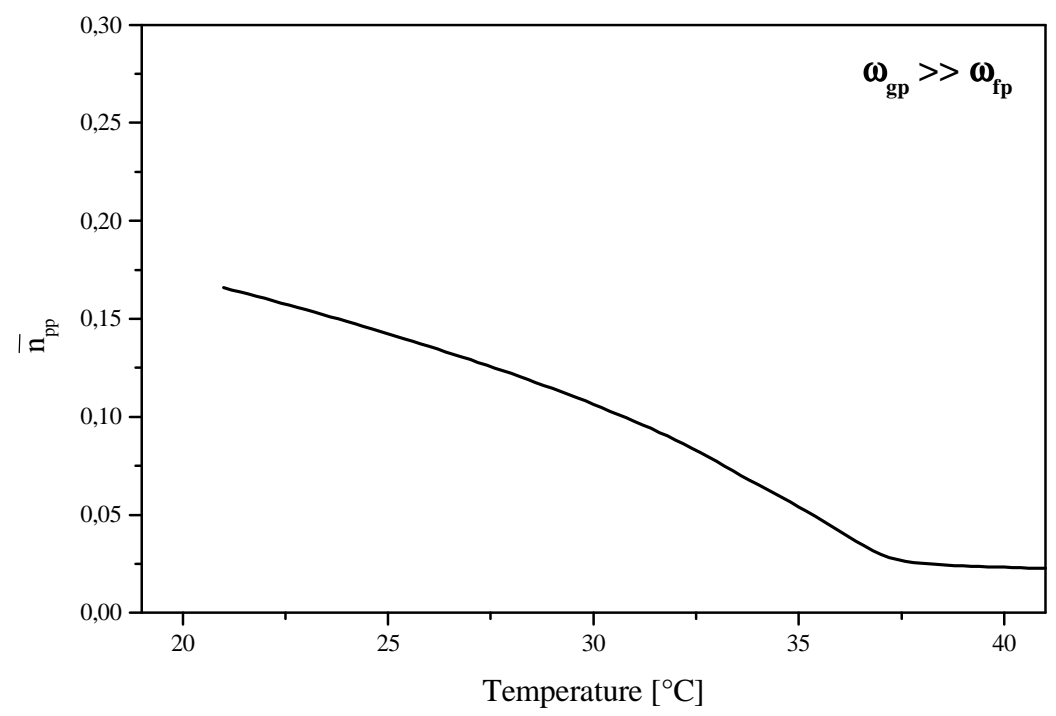

Figure 3.21. Calculated temperature profile of the cluster formation parameter, $\bar{n}_{p p}$, for a membrane containing $10 \mathrm{~mol} \%$ peptide which mixes well with the fluid phase, but demixes strongly from the gel lipid phase, $\omega_{f p}=0, \omega_{g p}=900 \mathrm{cal} / \mathrm{mol}$, $\omega_{g f}=315 \mathrm{cal} / \mathrm{mol}$.

Another example is alamethicin reconstituted into a planar bilayer membrane of 1-stearoyl3-myristoyl phosphatidylcholine. This unusual isomer of an asymmetric-chain phospholipid is capable of forming stable unsupported bilayers (black lipid membranes) in the gel phase. The formation of ion conducting pores by oligomerization of alamethicin monomers is very strongly concentration-dependent: the conductance depends on the $9^{\text {th }}$ to $10^{\text {th }}$ power of the alamethicin concentration [2]. Therefore, it can be expected that the membrane conductance changes strongly in the region of the bilayer chain-melting transition, if the degree of aggregation and hence the local alamethicin concentration depends on the lipid. This corresponds exactly to the observation by Boheim et al. [2] (Figure 3.22). The current density increases from a level corresponding to only 1 pore $/ \mathrm{cm}^{2}$ in the fluid phase at $34^{\circ} \mathrm{C}$ to that corresponding to approximately $10^{6}$ pores $/ \mathrm{cm}^{2}$ in the gel phase at $24^{\circ} \mathrm{C}$. 


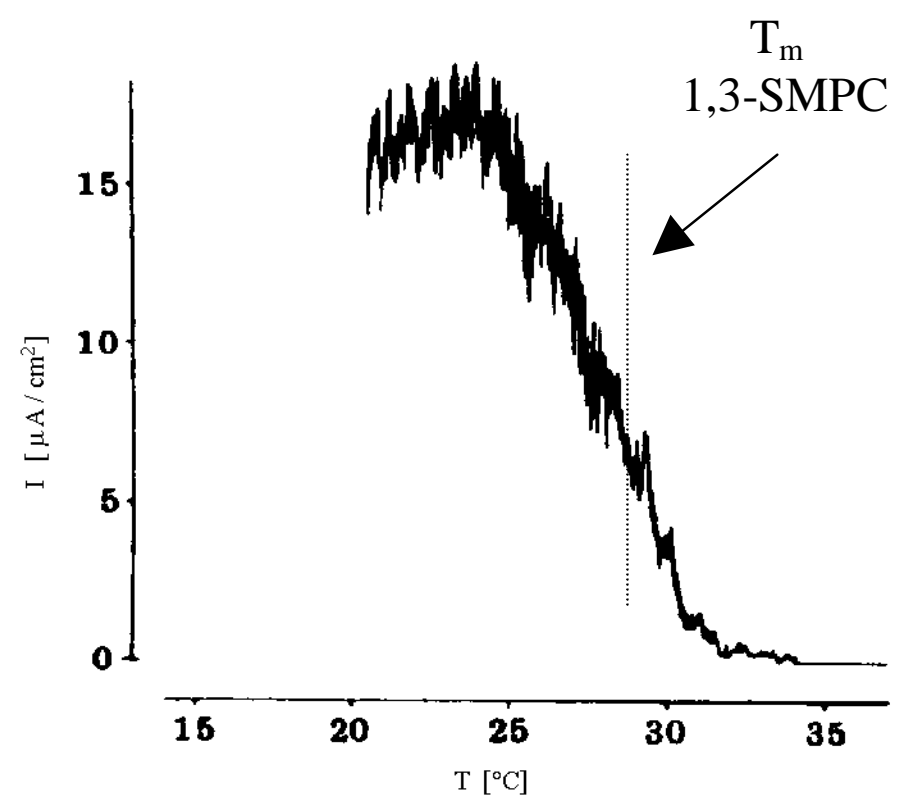

Figure 3.22. Temperature dependence of the transmembrane current density in bilayers of 1-stearoyl-3-myristoyl phosphatidylcholine (1,3-SMPC) containing alamethicin. The vertical line indicates the gel-to-fluid phase transition temperature of 1,3-SMPC bilayers. (Addapted from [2]).

The Monte Carlo simulations give an insight into the spatial distribution of the fluctuations within the membrane plane. In the demonstrated case of a lipid membrane containing peptides that aggregate in both lipid phases but have a preference to the fluid phase (Figure 3.18) it was shown that the fluctuations in the bilayer are not homogenous at all - the largest fluctuations are located at the lipid-peptide interface and they are extremely pronounced in the phase transition region. Since large fluctuations are related to high heat capacities (fluctuation-dissipation theorem) as well as to high compressibilities [11 and the references therein] and permeabilities [2], one may speculate that the presence of peptide causes local softening of the bilayer as well as permeability for water molecules and small ions.

It is interesting to note that in the lipid phase in which the peptide is not well soluble (in our case it is the gel phase), the large amplitude of the fluctuations at the lipid-protein interface might be important for ensuring the necessary freedom for protein conformations which is of biological importance. 


\subsection{Conclusions}

We adopted the two-state Ising model in order to describe the melting behavior of a lipid membrane with incorporated small integral protein. At fixed peptide fraction the model has only four variables, $\left(f, n_{g f}, n_{f p}, n_{g p}\right)$, which allows to construct a broad four-dimensional histogram for the density of states. The histogram technique is then used to calculate the heat capacity profiles of many model systems and to analyze experimentally measured $C_{p^{-}}$ traces of lipid-peptide mixtures.

From the shapes of the heat capacity profiles of DPPC and DMPC membranes containing gramicidin A we have suggested that the peptides aggregate in both lipid phases whereas the cluster formation in the gel phase is more pronounced. These predictions, based only on calorimetry measurements, were confirmed by AFM experiments on gramicidin A mixtures with DPPC and DMPC.

The model is, however, too simple to allow explaining each detail in the heat capacity profile of a mixed lipid-peptide system. For instance, it is not clear how to account for conformational changes of the peptide. One possibility would be to perform calculations with temperature dependent interaction energies, which is generally possible using the broad histogram approach. Another restriction of the histogram method is that it cannot account properly for the real size of a peptide. In fact, this is a general problem in lattice models. Since in experimental systems it is often observed that the peptide cross-section is somewhat larger than that of a lipid, this restriction makes the quantitative comparison between the MC calculations and the measured heat capacity traces difficult. The problem arises from the different change of the distributional entropy upon aggregation in the case of large and small peptides. Additionally, the finite size of the simulated matrix limits the size of the peptide cluster. The ratio between the minimal peptide-lipid interface and the system volume decreases with increasing size of the system. Thus, in the case of very pronounced peptide aggregation, larger matrices would provide more accurate heat capacity profiles.

Finally, the broad histogram technique links theory to experiment thus allowing a better analysis of measured heat capacity profiles. 


\section{Appendix}

We sampled four-dimensional histograms at the following parameters:

$$
\begin{aligned}
& f_{p}=n_{p} / n=0.1, \Delta H=8700 \mathrm{cal} / \mathrm{mol}, T_{m}=310.3 \mathrm{~K} \\
& \omega_{g f} \in\{300,315,330\} \mathrm{cal} / \mathrm{mol} \\
& \omega_{f p}, \omega_{g p} \in\{0,100,200,300,400,500,600,700,800,900\} \mathrm{cal} / \mathrm{mol}, \omega_{g p} \geq \omega_{f p} \\
& T \in\left\{\left(T_{m}-14\right), \ldots,\left(T_{m}+5\right)\right\}, \Delta T=1 \mathrm{~K}
\end{aligned}
$$

Under these conditions, the peptide interacts predominantly with the fluid lipid phase and (if an aggregation takes place) aggregates in the gel phase. Then, using the symmetry of the degeneracy (Equation (3.6)) we explored the part of the phase space, which corresponds the other case.

Each MC simulation contained $2 \cdot 10^{6} \mathrm{MC}$ cycles (complete random walk through the whole matrix) and took about $1 \mathrm{~h}$ on a Pentium III (700MHz) personal computer.

The matching method applied was just as the same as used to construct the broad twodimensional histogram (Section 2.5). The difficulty in this case results perhaps from the high dimensionality of the distributions. For instance, one can not draw a simple picture (as in Figure 2.4) to show the overlap between neighboring histograms. For the success of the matching procedure a good overlap between neighboring histograms is absolutely necessary. That is why the sampled probability distributions of states were matched together following a strict order:

1) T- scan: the histograms sampled at a constant set of interaction parameters, but different temperatures.

2) $\omega_{g p}$-scan: the histograms obtained at step 1) at constant $\omega_{f p}$ and $\omega_{g f}$.

3) $\omega_{f p}$-scan: the histograms constructed at step 2) at constant $\omega_{g f}$

4) $\omega_{g f}$-scan: the three histograms constructed at step 3).

For the sake of completeness we list the matching procedure in the four-dimensional case. Let's denote (for clarity) the three types of unlike nearest neighbors contacts by a vector $\vec{n}$ 
and the three interaction energies - by $\vec{\omega}$, i.e. $\vec{n}=\left(n_{g f}, n_{f p}, n_{g p}\right)$ and $\vec{\omega}=\left(\omega_{g f}, \omega_{f p}, \omega_{g p}\right)$. Then, the set of parameters is $\zeta=\left(T, \Delta H, T_{m}, \vec{\omega}\right)$. The excess free energy of a given configuration is expressed as

$$
\Delta G\left(n_{f}, \vec{n}, \zeta\right)=n_{f} \cdot(\Delta H-T \cdot \Delta S)+\vec{n} \cdot \vec{\omega}
$$

where $\vec{n} \cdot \vec{\omega}=n_{g f} \cdot \omega_{g f}+n_{f p} \cdot \omega_{f p}+n_{g p} \cdot \omega_{g p}$. The aim is to match the primary histogram $P\left(n_{f}, \vec{n}, \zeta\right)$ with the secondary distribution $P^{*}\left(n_{f}, \vec{n}, \zeta^{*}\right)$. Both histograms are normalized so that the sum of all probabilities is 1 .

The overlap is defined as the part of the phase space where

$$
P\left(n_{f}, \vec{n}, \zeta\right)>0 \text { and } P^{*}\left(n_{f}, \vec{n}, \zeta^{*}\right)>0
$$

The part of the overlapping region where the secondary distribution is more accurate than the primary one is $M^{4 d}$.

$$
P^{*}\left(n_{f}, \vec{n}, \zeta^{*}\right)>P\left(n_{f}, \vec{n}, \zeta\right) \text { for }\left\{n_{f}, \vec{n}\right\} \in M^{4 d}
$$

The secondary distribution is then recalculated using the parameters of the primary histogram, $\zeta^{*} \rightarrow \zeta$.

$$
P^{*}\left(n_{f}, \vec{n}, \zeta^{*}\right) \rightarrow \widetilde{P}^{*}\left(n_{f}, \vec{n}, \zeta\right)=\frac{P^{*}\left(n_{f}, \vec{n}, \zeta^{*}\right) \cdot \exp \left(\frac{\Delta G\left(n_{f}, \vec{n}, \zeta^{*}\right)}{R \cdot T^{*}}-\frac{\Delta G\left(n_{f}, \vec{n}, \zeta\right)}{R \cdot T}\right)}{\sum_{n_{f}, \vec{n}} P^{*}\left(n_{f}, \vec{n}, \zeta^{*}\right) \cdot \exp \left(\frac{\Delta G\left(n_{f}, \vec{n}, \zeta^{*}\right)}{R \cdot T^{*}}-\frac{\Delta G\left(n_{f}, \vec{n}, \zeta\right)}{R \cdot T}\right)}
$$

The recalculated distribution $\tilde{P}^{*}\left(n_{f}, \vec{n}, \zeta\right)$ is thus also normalized. A scaling ratio is determined in the following way:

$$
r=\frac{\sum_{\left\{n_{f}, \vec{n}\right\} \in M^{4 d}} P\left(n_{f}, \vec{n}, \zeta\right)}{\sum_{\left\{n_{f}, \vec{n}\right\} \in M^{4 d}} \widetilde{P}^{*}\left(n_{f}, \vec{n}, \zeta\right)}
$$

Then the primary histogram is improved and extended as follows:

1) if $\left\{n_{f}, \vec{n}\right\} \in M^{4 d}$, then $P\left(n_{f}, \vec{n}, \zeta\right)=r \cdot \widetilde{P}^{*}\left(n_{f}, \vec{n}, \zeta\right)$ 
2) if $\left\{n_{f}, \vec{n}\right\} \notin M^{4 d}$ and

2.1) $P^{*}\left(n_{f}, \vec{n}, \zeta^{*}\right)>P\left(n_{f}, \vec{n}, \zeta\right)$, then $P\left(n_{f}, \vec{n}, \zeta\right)=r \cdot \widetilde{P}^{*}\left(n_{f}, \vec{n}, \zeta\right)$

2.2) $P^{*}\left(n_{f}, \vec{n}, \zeta^{*}\right)<P\left(n_{f}, \vec{n}, \zeta\right)$, then $P\left(n_{f}, \vec{n}, \zeta\right)$ does not change.

3) normalization of the extended histogram.

Using this procedure and following the order of matching of the histograms described above, the overlap, $M^{4 d}$, covered in each case about $10 \%$ of the phase space explored by the single histogram (primary or secondary). It turns out that this is enough to calculate accurately the scaling ratio (see the comparison between histogram calculations and direct MC simulation results - Figure 3.5-3.7).

\section{References}

[1] Boheim, G. (1974). Journal of Membrane Biology. Vol. 19, 277-303

[2] Boheim, G., Hanke, W., and Eibl, H. J. (1980). Proc. Natl. Acad. Sci. USA, Vol. 77, 3403-3407

[3] Cruisero-Hansson, L., Ipsen, J. H., and Mouritsen, O. G. (1990). Biochimica et Biophysica Acta, Vol. 979, 166-176

[4] Dumas, F., Lebrun, M.-C., and Tocanne, J.-F. (1999). FEBS Letters, Vol. 458, 271277

[5] Fattal, D. R. and Ben-Shaul, A. (1993). Biophysical Journal, Vol. 65, 1795-1809

[6] Ferrenberg, A. M. and Swendsen, R. H. (1988). Physical Review Letters, Vol. 61, 2635

[7] Freire, E., Markello, T, Rigell, C., and Holloway, P. W. (1983). Biochemistry, Vol. 22, $1675-1680$

[8] Fox, R. O. Jr. and Richards, F. M. (1982). Nature, Vol. 30, 325-330 
[9] Gennis, R. B. (1989). Biomembranes. In Molecular Structure and Function, SpringerVerlag, New-York

[10] Halstenberg, S., Heimburg, T., Hianik, T., Kaatze, U., and Krivanek, R. (1998). Biophysical Journal, Vol. 75, 264-271

[11] Heimburg, T. (1998). Biochimica et Biophysica Acta, Vol. 1415, 147

[12] Heimburg, T. and Biltonen, R. L. (1996). Biophysical Journal, Vol. 70, 84

[13] Heimburg, T. and Marsh, D. (1993). Biophysical Journal, Vol. 65, 2408-2417

[14] Heimburg, T. and Marsh, D. (1996). in Biological Membranes: A molecular perspective from computation and experiment, edited by K. M. Merz and B. Roux (Birkhäuser, Boston), pp. 405-462

[15] Janiak, M. J., Small, D. M., and Shipley, G. G. (1976). Biochemistry, Vol. 15, 45754580

[16] Kappel, T., Anken, R. H., Hanke, W., and Rahmann, H. (2000). Cellular and Molecular Neurobiology, Vol. 20, 579-589

[17] Kawasaki, K. (1972). In Phase Transitions and Critical Phenomena, Vol. 2, edited by C. Domb and M. S. Green (Academic Press, London), pp. 443

[18] Keller, S. L., Bezrukov, S. M., Gruner, S. M., Tate, M. W., Vodyanoy, I., and Parsegian, V. A. (1994). Biophysical Journal, Vol. 65, 23-27

[19] Kurrle, A., Rieber, P., and Sackmann, E. (1990). Biochemistry, Vol. 29, 8274-8282

[20] Mobashery, N., Nielsen, C., and Andersen, O. S. (1997). FEBS Letters, Vol. 412, 1520

[21] Morrow, M. R., Davis, J. H., Sharom, F. J., and Lamb, M. P. (1986). Biochimica et Biophysica Acta, Vol. 858, 13-20

[22] Mou et al. (1996). Biochemistry, Vol. 35, 3222-3226

[23] Mouritsen, O. G. (1990). in Molecular description of biological membrane components by computer aided conformational analysis, edited by R. Brasseur (CRC Press, Boca Raton), pp. 3-83

[24] Mouritsen, O. G. and Biltonen, R. L. (1992). In Protein-Lipid Interactions, edited by A. Watts, Elsevier, New York, 1-35

[25] Mouritsen, O. G. and Bloom, M. (1984). Biophysical Journal, Vol. 46, 141-153 
[26] Mouritsen, O. G. and Bloom, M. (1993). Annu. Rev. Biophys. Biomol. Struct., Vol. 22, $147-171$

[27] Mouritsen, O. G. and Jørgsen, K. (1994). Chemical Physics of Lipids, Vol. 73, 3-25

[28] Mouritsen, O. G. and Sperotto, M. M. (1992). In Thermodynamics of cell surface receptors, edited by M. Jackson, CRC Press, Boca Raton, FL. 127-181

[29] Pink, D. A. and Chapman, D. (1979). Proc. Natl. Acad. Sci. USA, Vol. 76, 1542

[30] Pink, D. A., Green, T. J., and Chapman, D. (1980). Exp. Theor. Biochem., Vol. 19, 349-356

[31] Prenner, E. J., Lewis, R. N. A. H., Kondejewski, L. H., Hodges, R. S., and McElhaney, R. N., Biochimica et Biophysica Acta, Vol. 1417, 211-223

[32] Rodgers, W. and Glaser, M. (1993). Biochemistry, Vol. 32, 12591-12598

[33] Sperotto, M. M. and Mouritsen, O. G. (1991). Biophysical Journal, Vol. 59, 261-270

[34] Sperotto, M. M. and Mouritsen, O. G. (1993). European Biophysical Journal, Vol. 22, 323-328

[35] Sperotto, M. M., Ipsen, J. H., and Mouritsen, O. G. (1989). Cell Biophysics, Vol. 14, $79-95$

[36] Tanford, C. (1980). The hydrophobic effect: Formation of micelles and biological membranes, A Wiley-Interscience Publications, New York.

[37] Vodyanoy, I., Bezrukov, S. M., and Parsegian, V. A. (1993). Biophysical Journal, Vol. 65, 2097-2105

[38] Wooley, G. A., Epand, R. M., Kerr, I. D., Sansom, S. P., and Wallace, B. A. (1994). Biochemistry, Vol. 33, 6850-6858

[39] Zhang, Y.-P., Lewis, R. N. A. H., Hodges, R. S., and McElhaney, R. N. (1995), Biochemistry, Vol. 34, 2362-2371

[40] Zhang, Z., Sperotto, M. M., Zuckermann, M. J., and Mouritsen, O. G. (1993). Biochimica et Biophysica Acta, Vol. 1147, 154 


\section{Simulation study of the lipid kinetics}

\subsection{Introduction}

Close to the melting transition the relaxation in the lipid system following an external distortion becomes very slow. This phenomena has been investigated by various experimental methods. The transient techniques (e.g. temperature-, pressure-, pH-jump) apply a stepwise change of the control parameter to drive the system to a new equilibrium. Then an optical detection $[2,4,6,11,17,18]$ was used to monitor the relaxation of the lipid dispersion. The stationary methods imply disturbance of the system equilibrium by an oscillatory change of the perturbation parameter. A classical example of this technique is the measurement of sound dispersion and absorption [13,7] where compression and rarefaction pressure waves of sound propagation are responsible for perturbing a pressuredependent equilibrium. Using a volume-perturbation calorimeter, van Osdol et al. [19,20] measured the temperature and pressure responses to the volume perturbations in order to monitor the relaxation in vesicle dispersions.

The determined rates of the lipid relaxation are strongly temperature-dependent, displaying narrow maxima at the midpoint of the transition. In general, multilamellar vesicle dispersions display slower relaxation at the phase transition than unilamellar vesicles [20]. Thus, the relaxation has been found to depend on cooperativity.

In this chapter we present a Monte Carlo simulation study of the lipid kinetics based on the two-state Ising model. Since the Monte Carlo simulation approach exploits a scheme of stochastic dynamics, it is, in principle, impossible to obtain quantitative information about time-dependent processes from such calculations. However, the MC simulations suggest that the relaxation times and the heat capacity are closely related. To justify this finding we outline a simple theory thus deriving the relaxation rates from the two-dimensional histogram of density of states. Additionally, we show results from two type of kinetics experiments - frequency dependent calorimetry and pressure-calorimetry. 


\subsection{Monte Carlo simulations including a temperature-jump}

In comparison to the experimental difficulties in performing T-jump measurements, it is pretty easy to disturb the equilibrium in a MC simulation. One simply induces a sudden temperature change after a desired equilibration time and observes the response of the system. Figure 4.1 shows relaxation profiles of the fraction of fluid lipids after a single temperature-jump.

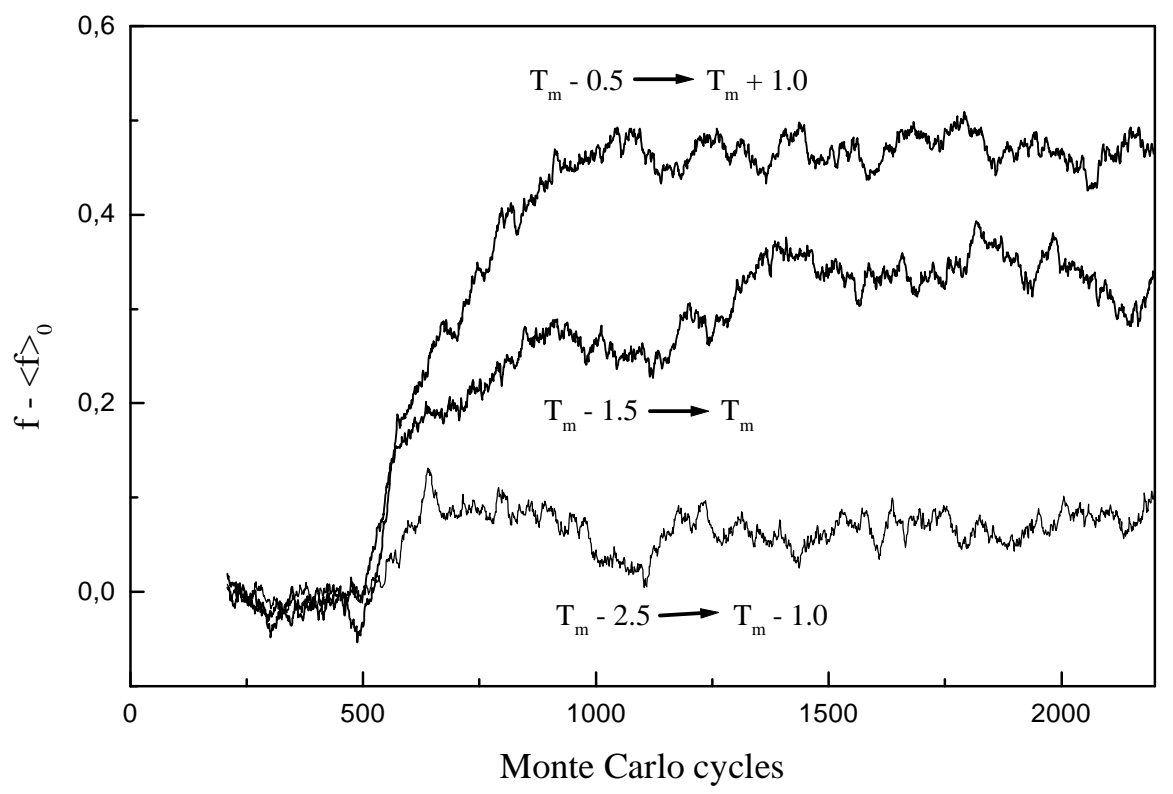

Figure 4.1. Relaxation of the fraction of fluid lipids after a temperature-jump in MC simulation. $\langle f\rangle_{0}$, the fluid fraction before the T-jump, is subtracted in order to compare the relaxation behavior in the three cases. The MC simulations were performed on a 31.31 matrix with periodic boundary conditions with the following parameters: $\Delta H=8700 \mathrm{cal} / \mathrm{mol}, \omega_{g f}=310 \mathrm{cal} / \mathrm{mol}$ and $T_{m}=310.3 \mathrm{~K}$.

In all these three cases the size of the temperature-jump is $1.5 \mathrm{deg}$. It is evident that jumping into the mid-point, $T_{m}$, of the gel-to-fluid phase transition is connected with an extremely slow relaxation (the red line). In principle, one can estimate the relaxation times of the system by performing MC simulations including a T-jump. However, because of the large magnitude of the fluctuations, one has to average the profiles obtained from several T-jumps at the same set of parameters (in Figure 4.1 we have shown non-averaged response profiles). In order to avoid this problem, we calculated the relaxation times from the decay of the autocorrelation function of the molar enthalpy of the system. 


\subsection{Calculation of the relaxation times in the two-state Ising model}

The fluctuations in the fraction of fluid lipids obtained from Monte Carlo simulations at three different temperatures are illustrated in Figure 4.2.

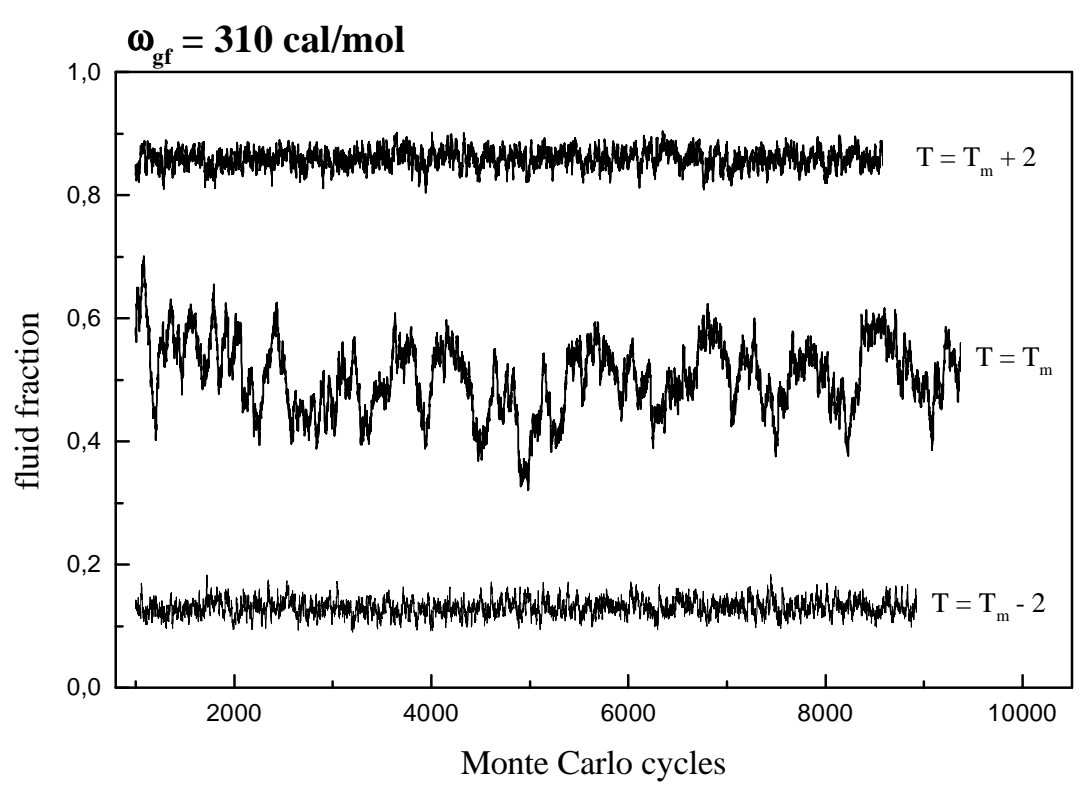

Figure 4.2. Fluctuations in the percentage of fluid lipids about the mean value over a range of $10000 \mathrm{MC}$ cycles. The initial 1000 simulation steps were discarded in order to ensure a representative configuration. The simulations were performed on a 31.31 matrix with periodic boundary conditions with the following parameters: $\Delta H=8700 \mathrm{cal} / \mathrm{mol}, \omega_{g f}=310 \mathrm{cal} / \mathrm{mol}$ and temperatures below (bottom), at (center) and above (top) $T_{m}=310.3 \mathrm{~K}$, corresponding to the mid-point of the gel-tofluid transition.

The thermal fluctuations are small deviations from equilibrium. From Figure 4.2 it becomes evident that the fluctuations at the transition temperature (the red line) have not only larger amplitude but also longer „lifetime“ then those at higher or lower temperatures. A measure for the amplitude of the fluctuations displays the heat capacity (Equation (2.6)). Quantitative information about the decay of the fluctuations gives their autocorrelation function. Since enthalpy and volume and to a first approximation the number of fluid lipids are proportional, it does not matter of which thermodynamic function the fluctuations are considered. Here we will consider the autocorrelation function of the molar enthalpy, 


$$
G(t)=\frac{\left\langle H\left(t^{\prime}+t\right) H\left(t^{\prime}\right)-\langle H\rangle^{2}\right\rangle}{\left\langle H\left(t^{\prime}\right)-\langle H\rangle\right\rangle^{2}}
$$

where \langle\rangle denotes time averages.

Three such autocorrelation functions at temperatures below, at and above the phase transition temperature, $T_{m}$, are shown in Figure 4.3.

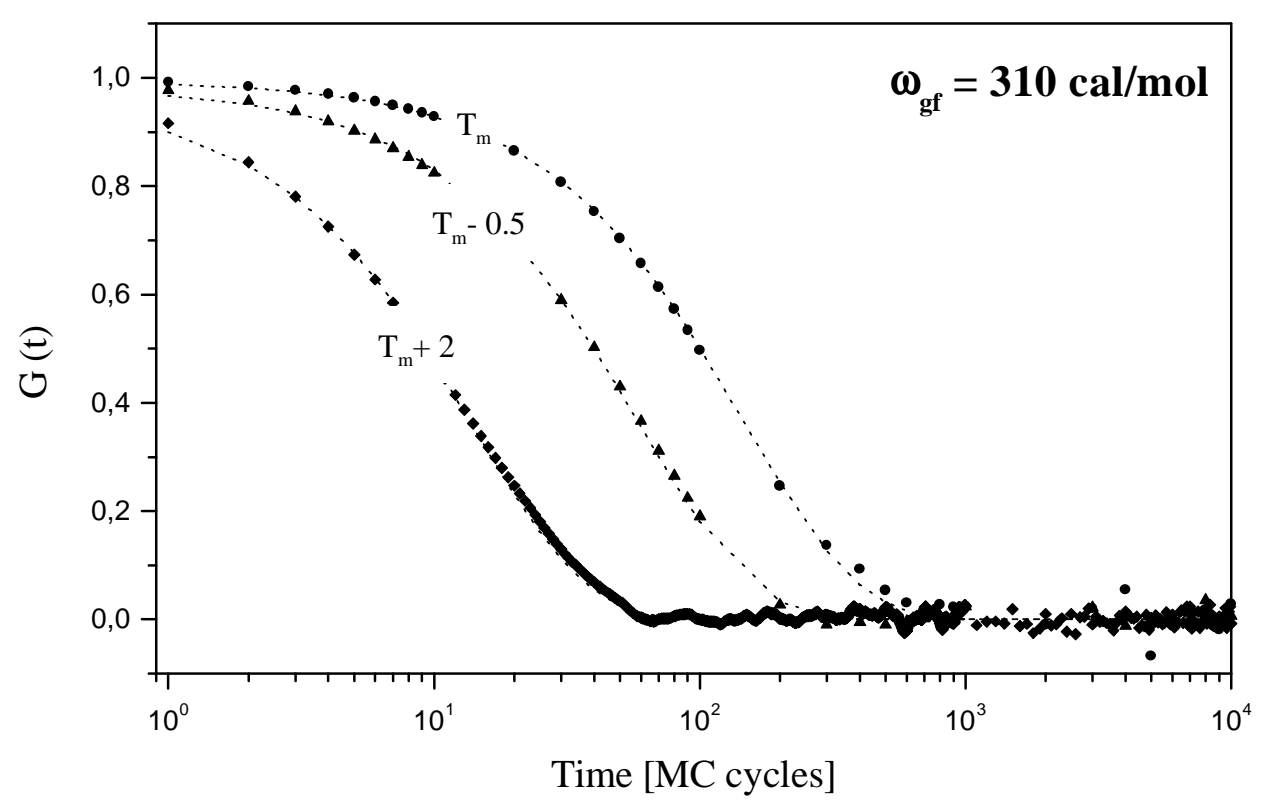

Figure 4.3. Autocorrelation function of the molar enthalpy for three temperatures: below $T_{m}$ (solid triangles), at $T_{m}$ (solid circles) and above $T_{m}$ (solid diamonds). The dotted lines represent single exponential decay fits in the three cases. Simulations on a 31.31 matrix with periodic boundary conditions with the following parameters: $\Delta H=8700 \mathrm{cal} / \mathrm{mol}, \omega_{g f}=310 \mathrm{cal} / \mathrm{mol}$ and $T_{m}=310.3 \mathrm{~K}$ (corresponding to DPPC large unilamellar vesicles). Each autocorrelation function is calculated over $10^{6} \mathrm{MC}$ cycles.

According to the point of view developed by Onsager [15], the decay of equilibrium fluctuations of the enthalpy (as well as other mechanical variables) can be described, on the average, by a sum of exponential decays. In our case the autocorrelation functions can be well approximated by a single exponential decay. The calculated relaxation times for systems with different cooperativities are given in Figure 4.4. The temperature dependence 
of the resolved relaxation rates is very similar to the heat capacity profile calculated at the same set of parameters from the broad two-dimensional histogram (Equation (2.9)). This phenomenon will be elucidated by a simple calculation, based on the histogram of the density of states.
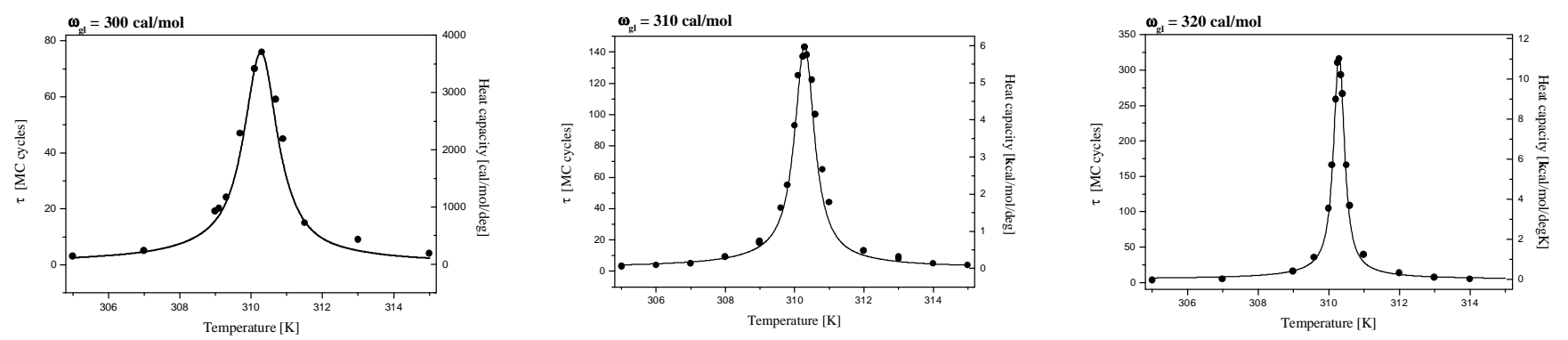

Figure 4.4. Relaxation times determined from the decay of the autocorrelation function of the molar enthalpy, and heat capacity profiles for three different cooperativities. Solid circles: relaxation times; Solid line: heat capacity profile. Left: $\omega_{g f}=300 \mathrm{cal} / \mathrm{mol}$; Center: $\omega_{g f}=310 \mathrm{cal} / \mathrm{mol}$; Right: $\omega_{g f}=320 \mathrm{cal} / \mathrm{mol}$.

\subsection{Why are the relaxation times closely related to the heat capacity?}

In the following we outline a simple theory [10] in order to justify the finding that the relaxation times resolved from the decay of the enthalpy fluctuations in a MC simulation and the heat capacity have closely related temperature dependencies. In this consideration we do not regard histograms displaying two peaks, i.e. we exclude the cases of high cooperativity near the melting temperature $T_{m}$ (see Figure 2.6).

As mentioned above (section 2.4), the partition coefficients in the two-dimensional Ising model are given by

$$
P\left(n_{f}, n_{g f}, \zeta\right)=\frac{\Omega\left(n_{f}, n_{g f}\right) \cdot \exp \left(-\Delta G\left(n_{f}, n_{g f}, \zeta\right) / R \cdot T\right)}{\sum_{n_{f}, n_{g f}} \Omega\left(n_{f}, n_{g f}\right) \cdot \exp \left(-\Delta G\left(n_{f}, n_{g f}, \zeta\right) / R \cdot T\right)}
$$

where $n_{f}$ is the number of fluid lipid molecules, $n_{g f}$ is the number of unlike nearest neighbor contacts and $\zeta$ denotes the set of parameters, $\left(T, \Delta H, T_{m}, \omega_{g f}\right)$. 
One can recalculate this distribution function in order to obtain the distribution of states with a given enthalpy, $P(H)$. The probability to find a state with a given value of the molar enthalpy is thus given by

$$
P(H)=\left.\sum_{n_{f}, n_{g f}} P\left(n_{f}, n_{g f}\right)\right|_{n_{f} \Delta H+n_{g f} \omega_{g f}=H}
$$

According to [12], the Gibbs free energy $G(H)$ for such a state can be expressed as

$$
G(H)=-\ln P(H)+C, C=\text { const }
$$

and, correspondingly, the entropy $S(H)$ is given by

$$
S(H)=\frac{H+\ln P(H)-C}{T}
$$

Since we consider here a histogram which has only one peak, the distribution function of the enthalpy can well be approximated by a Gaussian distribution (Figure 4.5)

$$
P(H)=P_{0} \exp \left(-\frac{\left(H-H_{0}\right)^{2}}{\sigma^{2}}\right)
$$

where $\left(H-H_{0}\right)$ are small fluctuations around the equilibrium value $H_{0}$.

Then the Gibbs free energy is simply a quadratic polynomial:

$$
G(H)=\frac{\left(H-H_{0}\right)^{2}}{\sigma^{2}}-\ln P_{0}+C
$$

and, consequently, the entropy in this case is given by a harmonic potential

$$
S(H)=\frac{H-\Delta G(H)}{T}=\frac{H}{T}-\frac{\left(H-H_{0}\right)^{2}}{T \cdot \sigma^{2}}-\frac{\ln P_{0}+C}{T}
$$


$\mathrm{T}_{\mathrm{m}}-5$
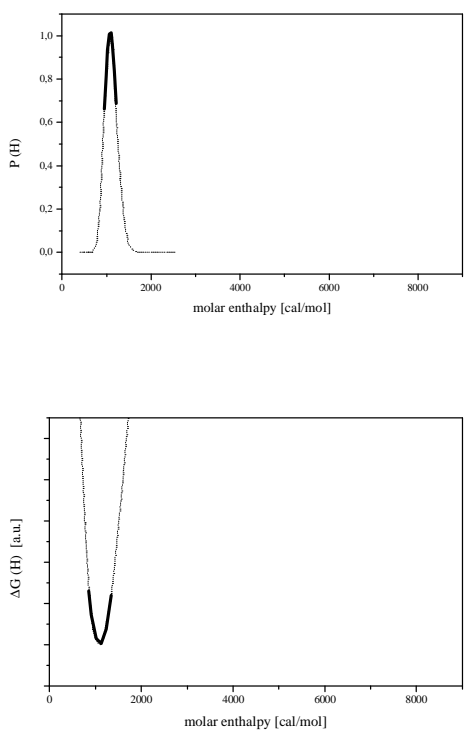

$\mathrm{T}_{\mathrm{m}}$
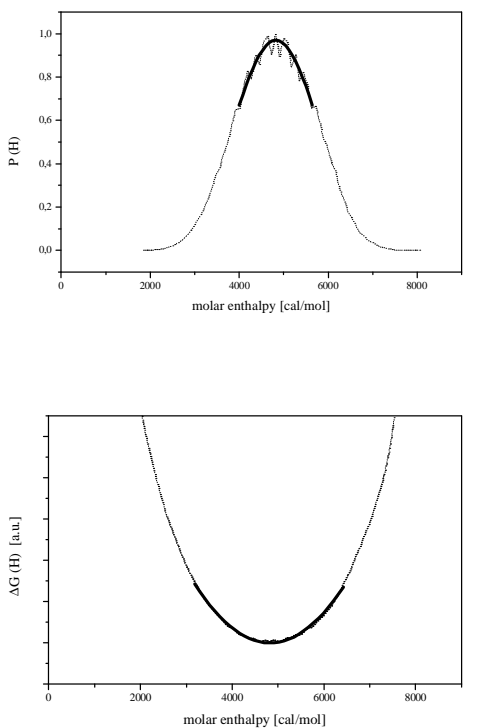

$\mathrm{T}_{\mathrm{m}}+5$
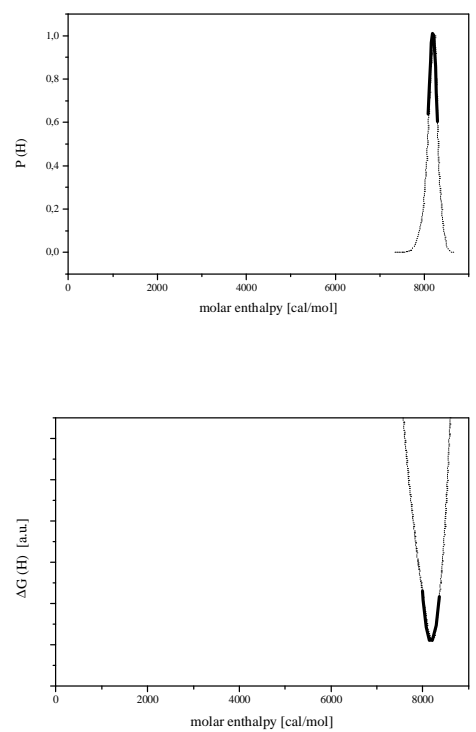

Figure 4.5. Distribution of states (top) and the corresponding Gibbs free energy (bottom). The profiles are derived from the broad two-dimensional histogram (Figure 2.4) and calculated at the following parameters: $\Delta H=8700 \mathrm{cal} / \mathrm{mol}$, $\omega_{g f}=300 \mathrm{cal} / \mathrm{mol}$ and temperatures below (left hand side), at (center) and above (right hand side) the phase transition temperature $T_{m}=310.3 \mathrm{~K}$. The thick black lines are Gaussian (top) and $2^{\text {nd }}$ order polynomial (bottom) fits for small deviation from equilibrium.

The thermodynamic forces that drive the system to the equilibrium state can be generally derived from the entropy [15]

$$
X_{i}=\sum_{j}\left(\frac{\partial^{2} S}{\partial \alpha_{i} \partial \alpha_{j}}\right) \alpha_{j}
$$

where $\alpha_{j}$ denote the fluctuations. In linear non-equilibrium thermodynamics the fluxes are linear functions of the respective thermodynamic forces, i.e.

$$
J_{i}=\frac{d \alpha_{i}}{d t}=\sum_{j} L_{i j} X_{j}
$$

where $L_{i j}$ are phenomenological coefficients. Then, for the thermodynamic force that drives the lipid systems to the state with maximum enthalpy we have 


$$
X_{H}=\frac{\partial^{2} S}{\partial\left(H-H_{0}\right)^{2}} \cdot\left(H-H_{0}\right)=-\frac{2\left(H-H_{0}\right)}{T \sigma^{2}}
$$

and for the corresponding flux

$$
J_{H}=\frac{d\left(H-H_{0}\right)}{d t}=-\frac{2 L}{T \sigma^{2}} \cdot\left(H-H_{0}\right)
$$

This expression leads to a single exponential time dependence for the relaxation of the fluctuation $\left(H-H_{0}\right)$

$$
\left(H(t)-H_{0}\right)=\exp \left(-\frac{2 L}{T \sigma^{2}} t\right) \equiv \exp \left(-\frac{t}{\tau}\right)
$$

with a relaxation time given by

$$
\tau=\frac{T \sigma^{2}}{2 L}
$$

On the other hand, the heat capacity is related to the standard deviation of the enthalpy fluctuations:

$$
C_{p}=\frac{\left\langle H^{2}\right\rangle-\langle H\rangle^{2}}{R T^{2}}=\frac{\sigma^{2}}{R T^{2}}
$$

and hence

$$
\tau=\frac{R T^{3}}{2 L} \cdot C_{p}
$$

Thus, it turns out that the relaxation time, $\tau$, and the heat capacity multiplied with the third power of the temperature, $C_{p} \cdot T^{3}$, are proportional functions. The phenomenological coefficient here, $L$, has to be determined by the experiment.

This calculation allows to make two important statements: (i) the distribution of states contains information about the system relaxation; (ii) the relaxation times of lipid systems can, in principle, be resolved from calorimetry experiments. 


\subsection{Experimental results and discussion}

The close relation between the relaxation times and the heat capacity, estimated in MC simulations, was tested in two kinds of experiments - frequency-dependent calorimetry and pressure-calorimetry.

\subsubsection{Frequency-dependent calorimetry ${ }^{\dagger}$}

In the frequency-dependent calorimeter [16], the heat provided by the Ni-film has to be absorbed by the sample. This is a time dependent process. Thus, with periodic heating $\left(Q=\frac{1}{2} \cdot R I_{0}^{2} \cdot(1+\cos \omega t)\right)$ the system response becomes frequency dependent. The observable in this experiment was the thermal effusivity (Section 1.4). (We remind that the effusivity is expressed as $\varepsilon=C_{p} \cdot \rho \cdot \kappa$, where $C_{p}, \rho$ and $\kappa$ denote the heat capacity, the mass density and the thermal conductivity, respectively.)

From Eqs. (1.8) and (1.9) one obtains

$$
\frac{\varepsilon(T, f)}{\varepsilon_{0}(f)}=\left(\frac{\frac{U^{(e m p t y)}(f)}{U(T, f)}-1}{\frac{U^{(e m p t y)}(f)}{U_{0}(f)}-1}\right)^{2}
$$

where $U$ and $U^{(e m p t y)}$ are the measured amplitudes of the $3 \omega / 2$-signal with and without sample, respectively; $T$ is the temperature and $f=\omega / 2 \pi$ is the frequency of the oscillating heat. The index, 0 " denotes the values well outside the transition region, i.e. for $\left|T-T_{m}\right| / T_{m}<<1$ (we have used the first data point in each temperature scan).

The results from these kinetics experiments are presented in Figure 4.6. (I am very grateful to Dr. W. Doster from the Institute for Solid State Physics E13, TU Munich, for letting me use his frequency-dependent calorimeter and to Dr. H. Leyser for his help in performing the measurements.)

\footnotetext{
${ }^{\dagger}$ These experiments were performed at the Institute for Solid State Physics E13, TU Munich.
} 


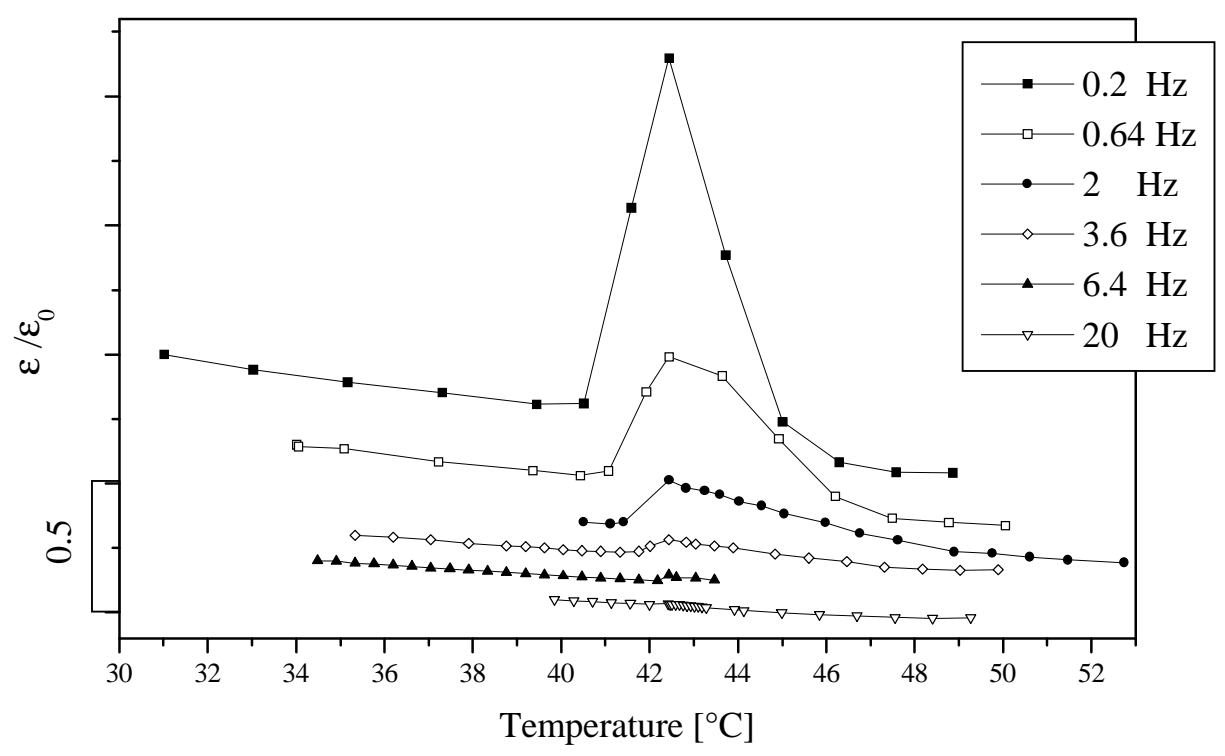

Figure 4.6. Temperature dependence of the normalized effusivity (Equation (4.18)) at different frequencies. Measurement on multilamellar vesicle dispersion of 100 $\mathrm{mM}$ DPPC in water.

At low frequencies the temperature profile of the effusivity (resp. heat capacity) displays a maximum. This maximum occurs close to the melting temperature of DPPC $(\sim 41 \mathrm{deg})$. However, since the amplitude of the temperature oscillations in the experiment was about 0.3 deg, i.e., much larger than the transition width ( $0.1 \mathrm{deg}$ - Figure 2.7 - 1.h.s.), the peak in the effusivity profile appears somewhat broadened. Its maximum decreases with increasing frequency which suggests that the decay of the enthalpy fluctuations in the system, which are in fact related to the chain melting, is in the order of seconds. On the other hand, the measurements demonstrate unambiguously that processes faster than $50 \mathrm{~ms}$ time scale (see the lowest curve) are for the multilamellar vesicle dispersion of adiabatic nature (no heat absorption).

These measurements are similar in spirit with the volume-perturbation experiments described in $[19,20]$. Since the basis of the frequency-dependent calorimetry is the absorption of heat by the sample, the kinetics studied is truly the kinetics of the lipid chains near the melting transition. However, the temperature control, as in other studies, remains a major problem. Therefore, the measurements allow only an estimation of the time scale of the relaxations in vesicle dispersions. 


\subsubsection{Relaxation times resolved from pressure-calorimetry}

The best experimental data about the lipid membrane relaxation near the chain melting transition were obtained in pressure-calorimetry measurements. This technique implies a pressure-jump whereas the DSC is used as a very good thermostat $[1,5]$.

Operating the calorimeter in an isothermal mode we performed pressure-jumps of +40 bar or -40 bar in order to alter the system equilibrium. Figure 4.7 shows a typical relaxation profile of the differential power provided by the calorimeter to the sample to establish the new equilibrium. In most cases this relaxation was interpreted as a single exponential decay (details about the technique and the data analysis will be given in [5]).

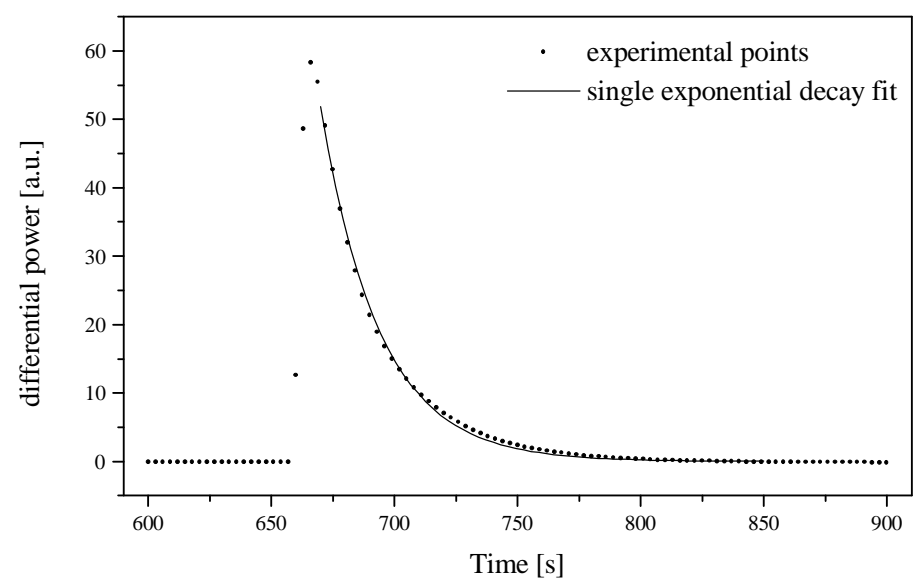

Figure 4.7. Relaxation profile (solid circles) after a pressure-jump of -40 bar, at temperature of $24.6^{\circ} \mathrm{C}$. The investigated system was MLV dispersion of $100 \mathrm{mM}$ DMPC in buffer (5 mM Hepes, $1 \mathrm{mM}$ EDTA, $\mathrm{pH}$ 7.7). From the single exponential fit (solid line) a relaxation rate of $24 \mathrm{~s}$ was resolved.

A comparison between the determined relaxation times and the heat capacity profile measured on the same sample is given in Figure 4.8. (I am very grateful to Peter Grabitz from our lab for letting me use his data sets for DPPC and DMPC prior detailed publication.) The relaxation times at temperatures below and above $T_{m}$ were measured with positive and negative pressure-jumps, respectively.

The rate constant in this regime is a convolution of the relaxation rate of the lipid dispersion with the time constant of the calorimeter. We have not yet determined the response function of the calorimeter itself. Therefore we show the row numbers with a baseline at about $5 \mathrm{~s}$.

\footnotetext{
${ }^{\ddagger}$ Here I present detailed view of unpublished data from Peter Grabitz, Göttingen, with permission.
} 


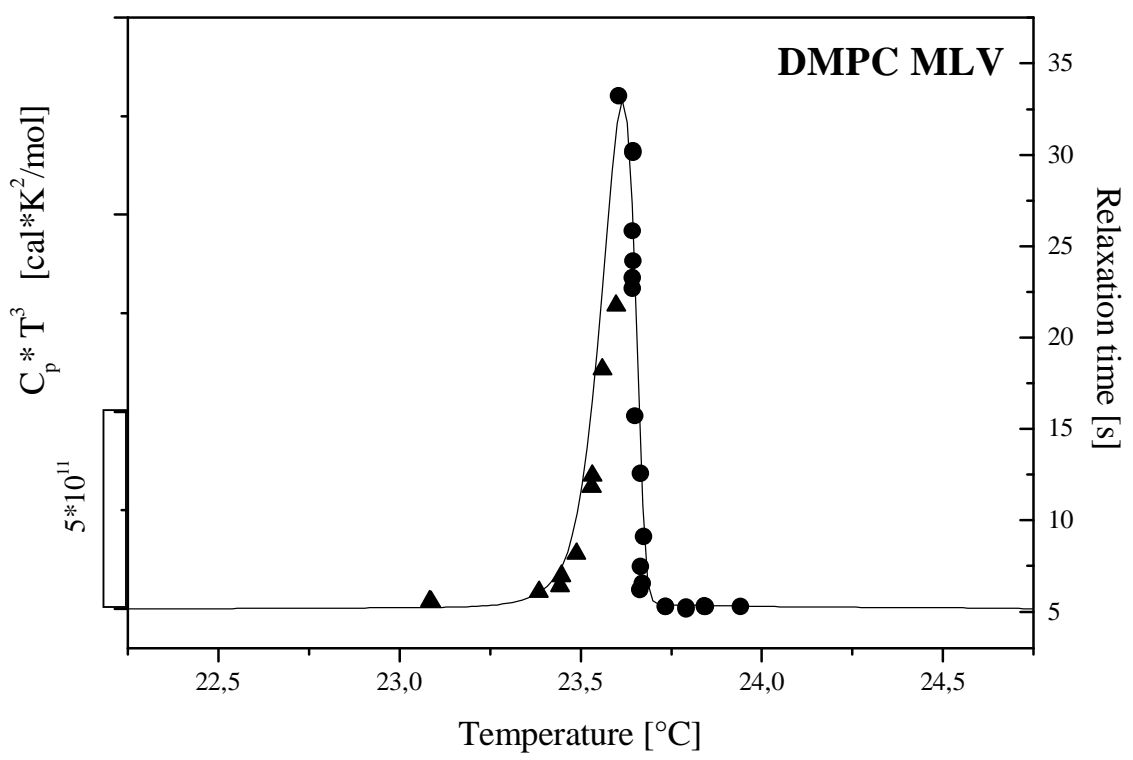

Figure 4.8. Relaxation times resolved by pressure-calorimetry and heat capacity of a MLV dispersion of $100 \mathrm{mM}$ DMPC in buffer (5 mM Hepes, $1 \mathrm{mM}$ EDTA, pH 7.7). Solid triangles: relaxation times, obtained after pressure-jumps of +40 bar; Solid circles: results from pressure-jumps of -40 bar; Solid line: heat capacity profile (multiplied with $T^{3}$ ) measured on the same sample.

The pressure-calorimetry experiments display the same close relation between the relaxation time and the heat capacity, as predicted from MC simulations and by the histogram calculations (see the previous section). This vesicle preparation displays a slightly asymmetric heat capacity profile and it is worth noting that the same asymmetry can be observed in the temperature dependence of the relaxation time (compare the low- and the high-temperature end of the transition). Thus, one may conclude that information about the kinetics of the membrane system can, in principle, be derived from a calorimetric measurement.

The results from the experiments performed on two other vesicle dispersions are given in Figure 4.9. The kinetics measurements in this case included only positive pressure-jumps. In the case of LUV dispersions, the data are somewhat scattered due to the low signal-to-noise ratio. 

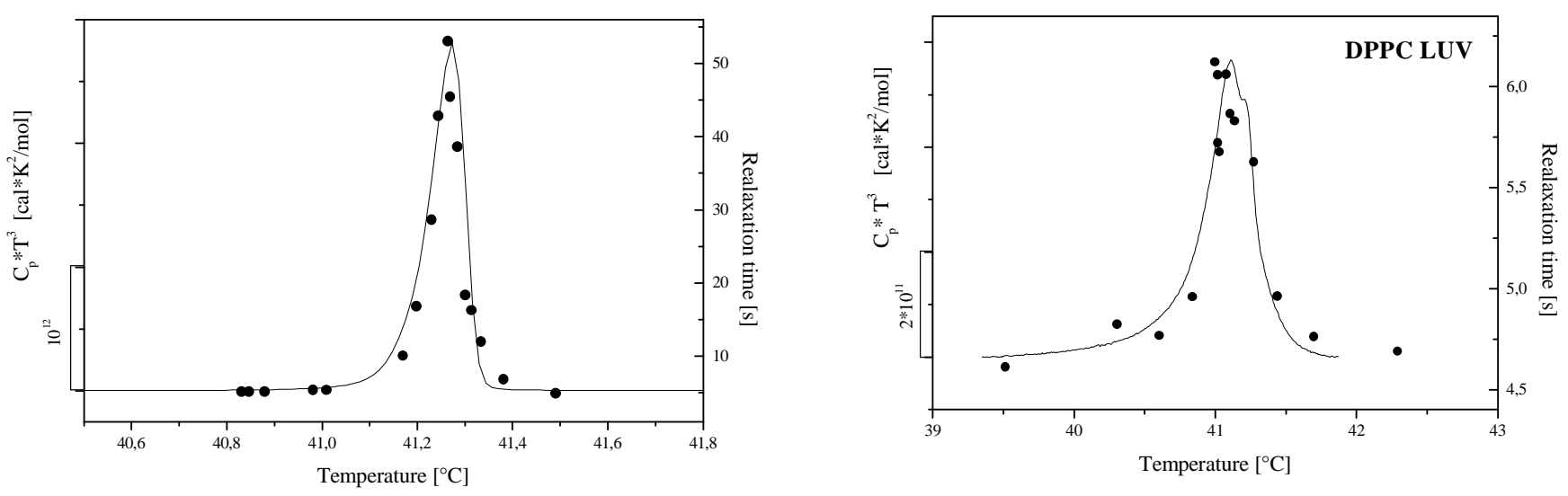

Figure 4.9. Relaxation times resolved by pressure-calorimetry and heat capacity of vesicle dispersions of $100 \mathrm{mM}$ DPPC. Left: MLV; Right: LUV. The relaxation times were obtained after pressure-jumps of +40 bar. Solid circles: relaxation times; Solid line: heat capacity profile (multiplied with $T^{3}$ ).

We would like to mention that these are by far the best relaxation data on lipid dispersions available, mainly because of the very stable temperature control during the experiment $(\sim 0.001 \mathrm{deg})$. This is of crucial importance especially when studying the kinetics of very cooperative transitions like in the case of multilamellar vesicle dispersions (Figure 4.8 and Figure 4.9-left hand side). Moreover, since this experimental technique measures the relaxation of the system enthalpy, the rate constants resolved are truly related to the chainmelting process.

\subsubsection{Discussion}

A general finding from the various temperature-jump [18], pressure-jump [2] and volumeperturbation [20] techniques has been that the main transition is characterized by several relaxation times (up to five [4]) in the range from nano-seconds to seconds. The processes in the time regime miliseconds to seconds have been suggested as being cooperative processes involving a large number of molecules via cluster formation and cluster melting [4]. The smaller rate constants are generally dependent on the detection technique used to monitor the lipid relaxation and are related to single-molecule effects like head group orientation [19]. The latter are processes that lead to a very small change of the system energy.

Computer simulations based on the ten-state Pink model demonstrated that the relaxation towards equilibrium is slowed down by the intermediate chain conformational states which 
are found to have a tendency to appear on the surface of the lipid domains formed near the transition [14]. This so-called softening of the interfaces implies that the interaction between the domains is screened which effectively slows down the tendency for the domains to fuse and form a new phase.

In [7] it was shown that the ultrasonic velocities measured in DMPC and DMPC/cholesterol mixtures display a very good correlation with the heat capacity profiles. This finding was explained in a thermodynamic theory using the proportionality between enthalpy and volume changes near the lipid chain-melting transition [8].

We studied the kinetics of the lipid monolayer with the help of Monte Carlo simulations based on the two-state Ising model. The calculated autocorrelation function of the molar enthalpy, which gives information about the decay of the thermal fluctuations, can well be approximated with a single exponential decay function. We found empirically that the relaxation times calculated by simulations are closely related to the heat capacity. By means of a simple theory and use of the broad two-dimensional histogram of distribution of states, this finding was justified. Thus, it turns out that the degeneracy of states sampled in a MC simulation can be used to obtain information about the kinetics of the melting transition of the lipid system. From the broad histogram one can derive the distribution of enthalpy states for a given set of parameters. When this distribution has a Gauss-like shape, the relaxation of the fluctuations is given by a single exponential decay and the characteristic time is simply related to the width of the Gauss distribution.

In the pressure-calorimetry experiments we observed a single exponential decay of the enthalpy fluctuations even at temperatures close to the heat capacity maximum. Indeed these precise measurements agree very well with our theoretical calculations thus suggesting that the simple two-state Ising model of the lipid membrane is enough to describe the cooperative effects at the chain-melting transition. Moreover, from a calorimetric measurement only one is able, in principle, to deduce information about the kinetics of the membranous system.

Let's assume that the phenomenological coefficient $L$ in Eq. (4.16) does not depend on the type of lipid. Generally, a correct statement on this parameter can only be given when the relaxation process is separated from the response function of the calorimeter. As mentioned above, the time constant of the calorimeter is supposed to be in the order of $5 \mathrm{~s}$, thus much smaller than the maximal relaxation time resolved in the case of multilamellar vesicles. 
Therefore, using the experimental data for MLV dispersion of DPPC at $T_{m}$, one calculates $L=5.28 \cdot 10^{10}\left[\frac{c a l^{2} \cdot K}{m o l^{2}}\right]$. Then, using this value the relaxation time (at $T_{m}$ ) for DMPC can be estimated. From Equation (4.16) one obtains $\tau^{\text {calc. }}=24.26 \mathrm{sec}$. which is in the same order of magnitude as the measured time constant of $33.2 \mathrm{sec}$. Bearing in mind the simplicity of our model, the predicative power of the method is pretty good. Thus, we conclude that from a calorimetric measurement it is, in principle, possible to estimate the time scale of the lipid membrane relaxation.

In Chapter 2, we applied the histogram technique to generate heat capacity profiles at various sets of parameters and to fit experimentally measured $C_{p}$-traces. Here, it was demonstrated that the broad monolayer histogram contains the information necessary to resolve relaxation properties of the chain melting process. The latter is the thermodynamically relevant part of the lipid main transition since it is connected with the greatest energy change $(8700 \mathrm{cal} / \mathrm{mol}$ in the case of DPPC).

\subsection{Conclusions}

We performed a Monte Carlo simulation study of the lipid kinetics near the gel-to-fluid transition. Our considerations are based on the two-state Ising model, thus accounting for the cooperative effects accompanying the chain-melting process, but neglecting the changes occurring on the level of individual molecules. Therefore, the rate constants determined from the decay of the enthalpy fluctuations characterize the relaxation of the lipid chains. These characteristic times are very closely related to the heat capacity, as suggested from the MC simulations. We developed a theory in order to justify this finding, thus deriving the relaxation times from the broad distribution of states. The results from the pressurecalorimetry experiments, which are by far the best relaxation data on lipid dispersions available, are in very good agreement with the theoretical calculations. Thus, we conclude that from a heat capacity measurement one can, in principle, deduce information about the relaxation of the lipid chains near the melting transition. 


\section{References}

[1] Ebel, H.F. (1999). Graduate thesis, Georg-August-Universität, Göttingen. Kalorische und strukturelle Umwandlungen von Lipid-Membranen: Koplung von Wärmekapazität und Volumenexpansionskoeffizienten.

[2] Elamrani, K. and Blume, A. (1983). Biochemistry, Vol. 22, 3305-3311

[3] Freire, E. and Biltonen, R. L. (1978). Biochimica et Biophysica Acta, Vol. 514, 54-68

[4] Genz, A. and Holzwarth, J. F. (1986). European Biophysical Journal, Vol. 13, 323330

[5] Grabitz, P. (2000). Graduate thesis, Georg-August-Universität, Göttingen. This work is in preparation and will be submitted at the end of 2000.

[6] Gruenewald, B., Blume, A., and Watanabe, F. (1980). Biochimica et Biophysica Acta, Vol. 597, 41-52

[7] Halstenberg, S., Heimburg, T., Hianik, T., Kaatze, U., and Krivanek, R. (1998). Biophysical Journal, Vol. 75, 264-271

[8] Heimburg, T. (1998). Biochimica et Biophysica Acta, Vol. 1415, 147

[9] Heimburg, T. and Marsh, D. (1996). in Biological Membranes: A molecular perspective from computation and experiment, edited by K. M. Merz and B. Roux (Birkhäuser, Boston), pp. 405-462

[10] Ivanova, V. and Heimburg, T. (1999). Poster at the Meeting of the German Biophysical Society.

[11] Kanehisa, M. I. and Tsong, T. Y. (1978). Journal of the American Chemical Society, Vol. 100, 424-432

[12] Lee, J. and Kosterlitz, J. M. (1991). Physical Review B, Vol. 43, 3265-3277

[13] Mitaku, S., Jippo, T., and Kataoka, R. (1983). Biophysical Journal, Vol. 42, 137-144

[14] Mouritsen, O. G. (1990). in Molecular description of biological membrane components by computer aided conformational analysis, edited by R. Brasseur (CRC Press, Boca Raton), pp. 3-83 
[15] Reichl, L. E. (1980). A Modern Course in Statistical Physics. University of Texas Press, Austin.

[16] Settles, M., Post, F., Müller, D., Schulte, A., and Doster, W. (1992). Biophysical Chemistry, Vol. 43, 107-116

[17] Strehlow, U. and Jähnig, F. (1981). Biochimica et Biophysica Acta, Vol. 641, 301310

[18] Tsong, T. Y. and Kanehisa, M. I. (1977). Biochemistry, Vol. 16, 2674-2680

[19] van Osdol, W.W., Biltonen, R. L., and Johnson, M. L. (1989). Journal of Biochemical and Biophysical Methods, Vol. 20, 1-46

[20] van Osdol, W. W., Johnson, M. L., Qiang Ye, and Biltonen, R. L. (1991). Biophysical Journal, Vol. 59, 775-785 


\section{General conclusion}

The thesis is dedicated to the investigation of the lipid-protein interactions both experimentally, by means of calorimetry, and theoretically, by means of Monte Carlo simulations. The main purpose was to link theory and experiment to enable statements on the thermotropic behavior of mixed lipid-peptide systems.

The theory is based on the two-state Ising model of which the distribution of states was explored by Monte Carlo simulations. In spite of its simplicity, the model contains the basic physics underlying the cooperativity of the lipid melting transition and the domain formation within the membrane plane. The Monte Carlo simulation approach allows to account properly for the thermal fluctuations in the lipid system. The greatest advantage of the two-state Ising model is its direct relation to the experiment. All parameters can be obtained in a calorimetric measurement. The unknown effective interaction parameter, $\omega_{g f}$, is derived indirectly from the peak width of the heat capacity. Since the Monte Carlo technique, as any other computer simulation method, is a very time consuming procedure, a quantitative comparison between calculations and experiment is largely facilitated by using the histogram method for analysis of the MC data. The histogram technique thus allows a quasi-analytical determination of mean values of various physical observables like enthalpy, volume, heat capacity, compressibility and others.

We sampled in one broad histogram the complete information of the degeneracy of states of a single-component lipid monolayer system. The histogram method for Monte Carlo data analysis was first applied to generate fits of experimentally measured heat capacity profiles of three different vesicle preparations. Thus, we have shown that the two-state model is good enough to describe the melting process in lipid dispersions with different cooperativities. The broad two-dimensional histogram even contains the information necessary to derive the heat capacity traces of a simple mixed lipid-peptide system - the case of gel-like peptides (section 3.3). The thermotropic behavior of this mixture was first resolved using the monolayer histogram technique.

In the general case of a lipid membrane incorporating small peptides, two component Monte Carlo simulations were performed. The peptide aggregation within the membrane plane results from the different solubility in the gel and the fluid phase. The information 
about the density of states in this case was sampled in a broad four-dimensional histogram. The construction of the latter is computationally more demanding which is the reason for considering only the case of a membrane containing 10 mol\% peptides. The broad fourdimensional histogram is used to calculate the heat capacity traces of various model mixtures. This model results can be used for a fast analysis of measured $C_{p}$-traces on lipidpeptide systems.

From the comparison of the theoretical calculations to the experimentally measured heat capacity profiles, the aggregation of the peptide gramicidin A in the gel and the fluid phase of DPPC and DMPC bilayer was predicted. These predictions were tested by means of the atomic force microscopy technique and a good agreement was found. Thus, we demonstrated that from calorimetric measurements only it is, in principle, possible to resolve peptide aggregation properties.

Since the histogram approach permits quasi-analytical Monte Carlo calculations, it opens a possibility for a theoretical investigation of various model cases with temperaturedependent interaction parameters. These are important cases since for some peptides it is said that they change their conformations upon bilayer melting. Without the use of the histogram technique, the calculation of heat capacity traces with temperature-dependent interaction energies for comparing them with experimentally measured profiles is virtually unfeasible.

We, furthermore, used the information sampled in the monolayer histogram to resolve lipid relaxation properties near the chain-melting transition. Since in our model only two lipid states, gel and fluid, are considered, the determined rate constants result truly from the cooperative processes of cluster formation and cluster melting. The relaxation times resolved characterize the decay of the enthalpy fluctuations. These are the thermodynamically relevant rate constants, since the chain-melting process is the reason for the large enthalpy change upon gel-to-fluid transition. Other relaxations related to singlemolecule effects like head group rotation etc., are not included in the two-state model. Moreover, the latter processes contribute very little to the change of the bilayer energy upon melting.

The proportionality between relaxation time and heat capacity, $\tau \propto C_{p} \cdot T^{3}$, estimated theoretically was tested in pressure-calorimetry experiments. The rate constants resolved by 
means of pressure-calorimetry are by far the best relaxation data available on lipid dispersions. This experimental technique measures the amount of heat provided to the sample after a pressure-jump to keep the sample temperature constant. Thus, pressurecalorimetry resolves the characteristic time of the enthalpy fluctuations. The results from these very precise measurements agree well with our theoretical calculations.

The relation between relaxation time and heat capacity opens a possibility for the derivation of information about the membrane kinetics from a simple calorimetric measurement. Thus, since the heat capacity profiles of lipid-peptide mixtures are often asymmetrically broadened and shifted, we predict that the relaxation times characterizing the decay of the enthalpy fluctuations in these systems display similar temperature dependence. Pressurecalorimetry experiments on mixed systems are under work. 


\section{LEBENSLAUF}

15.11.1972 Geboren in Vratza, Bulgarien

Nationalität: $\quad$ bulgarisch

\section{$\underline{\text { AUSBILDUNG }}$}

$9 / 79-9 / 86$

Grund- und Hauptschule in Krivodol, Bulgarien;

$9 / 86-6 / 90$

Mathematisches Gymnasium, Vratza, Bulgarien;

$10 / 90-7 / 95$

Studium an der Universität Sofia, Bulgarien;

Physik mit Schwerpunkt Theoretische Physik und Festkörperphysik;

$10 / 95-7 / 97$

European Masters Degree Course in „Purification and Control of Waste Waters,,, TEMPUS Programme JEP - 09789

seit $11 / 97$

Wissenschaftliche Mitarbeiterin am Max-Planck-Institut für biophysikalische Chemie, Arbeitsgruppe Thermodynamik von Membranen, Göttingen 